\title{
FUNCTIONAL AND MECHANISTIC CHARACTERIZATION OF THE F-BOX PROTEIN FBXW5
}

\author{
PhD Thesis \\ in partial fulfilment of the requirements \\ for the degree "Doctor rerum naturalium" \\ in the Molecular Biology Program \\ at the Georg August University Göttingen, \\ Faculty of Biology
}

submitted by

Achim Werner

born in

Kassel

Göttingen 2010 



\section{Declaration}

I hereby declare that this submission is my own work and that, to the best of my knowledge and belief, it contains no materials previously published or written by another person nor material which to a substantial extent has been accepted for the award of any other degree of the university or other institute of higher education, except where due acknowledgment has been made in the text.

Signature

Name

Date and Place 


\section{TABLE OF CONTENTS}

$\begin{array}{ll}\text { LIST OF TABLES } & \text { 7\# }\end{array}$

LIST OF FIGURES 7\#

ACKNOWLEDGEMENTS 9\#

LIST OF PUBLICATIONS

$\begin{array}{ll}\text { ABSTRACT } & 12 \#\end{array}$

INTRODUCTION 13\#

1.\# Ubiquitin and its conjugation to proteins 13\#

2.\# Forms of ubiquitylation 15\#

3.\# Functional outcomes of ubiquitylation 15\#

3.1.\# Degradation functions of Ubiquitin 16\#

3.1.1.\# Proteasome-dependent degradation 16\#

3.1.2.\# Lysosome-dependent degradation 16\#

3.2.\# Non-degradation functions of Ubiquitin 18\#

4.\# Regulating target specificity of ubiquitylation - E3 ligases 20\#

4.1.\# Types of Ubiquitin E3 ligases 20\#

4.2.\# Cullin-RING based Ubiquitin E3 ligases (CRLs) 22\#

4.2.1.\# Architecture of CRL complexes 22\#

4.2.2.\# General mechanisms of CRL complex regulation 22\#

4.2.3.\# Skp1-Cul1-F-box (SCF) Ubiquitin E3 ligases 26\#

5.\# F-box proteins 28\#

5.1.\# Classification of F-box proteins 28\#

5.2.\# Target recognition by F-box proteins 28\#

5.3.\# Physiological roles of F-box proteins 29\#

6.\# Fbxw5 - an unusual F-box protein 30\#

7.\# Aims of this work 32\#

MATERIALS AND METHODS 33\#

1.\# Materials 33\#

1.1.\# Technical equipment and software 33\#

1.2.\# Consumables 34\#

1.3.\# Chemicals, reagents and enzymes 34\#

1.4.\# Kits 36\#

1.5.\# Buffers and stock solution 37\#

1.6.\# Media 39\# 
1.4.\# Cell lines $\quad 40 \#$

1.5.\# Oligonucleotides $\quad 41 \#$

1.6.\# Vectors and plasmids $\quad 42 \#$

1.7.\# Peptides 45\#

1.8.\# Recombinant proteins $\quad$ 45\#

1.9.\# Antibodies 45\#

1.10. \#siRNAs 46\#

2.\# Methods 47\#

2.1.\# Molecular biology techniques $47 \#$

2.1.1.\# Preparation of chemically competent bacteria 47\#

2.1.2.\# Transformation of competent bacteria 48\#

2.1.3.\# Plasmid DNA preparation 48\#

2.1.4.\# Cloning 49\#

2.1.5.\# Sequencing of DNA 51\#

2.1.6.\# Plasmids constructed in this work

2.2.\# Biochemical methods 54\#

2.2.1.\# Measurement of protein concentration 54\#

2.2.2.\# SDS PAGE and detection of proteins 54\#

2.2.3.\# Production of recombinant proteins in bacteria 56\#

2.2.4.\# Production of recombinant proteins in insect cells $61 \#$

2.2.5.\# Production of recombinant proteins in mammalian cells $63 \#$

2.2.6. \# In vitro reconstitution of SCF ${ }^{\text {Fbxw5 }}$ 65\#

2.2.7.\# In vitro phosphorylation and dephosphorylation reactions 65\#

2.2.8.\# In vitro ubiquitylation reactions with recombinant proteins 65\#

2.2.9.\# Production and affinity purification of rabbit polyclonal Fbxw5 antibodies $66 \#$

2.2.10. Mammalian cell lysate and extract preparation 67\#

2.2.11.\#mmunoprecipitation of endogenous proteins 68\#

2.2.12. \#ass spectrometry analysis 69\#

2.3.\# Cell biological techniques $70 \#$

2.3.1.\# Cultivation of adherent and suspension cells $70 \#$

2.3.2.\# Transient transfection of mammalian cells 71\#

2.3.3.\# Generation and selection of stable flag-Fbxw5 HEK 293T cells 72\#

2.3.4.\# Manipulation of degradation pathways in mammalian cells $72 \#$

RESULTS 73\#

1.\# Fbxw5 assembles into SCF and CRL4 complexes 73\#

1.1.\# Fbxw5 predominantly assembles into SCF complexes 73\#

2.\# Fbxw5 interacts with Eps8 and Nek9 77\#

2.1.\# Mass spectrometry and western blot analysis of Fbxw5 immunoprecipitates reveal novel Fbxw5 interactors 77\#

2.2.\# Fbxw5 endogenously interacts with Eps8 and Nek9 82\# 
2.3.\# Fbxw5 interacts with Nek9 and Eps8 independently of its F-box domain 83\#

3.\# Eps8 is a substrate of SCF ${ }^{\text {Fbxw5 }}$ 85\#

3.1.\# Fbxw5 regulates Eps8 levels in vivo 85\#

3.2. \# SCF ${ }^{\mathrm{Fbxw5}}$ ubiquitylates Eps8 in vitro 89\#

3.3.\# Fbxw5-mediated ubiquitylation of Eps8 does not seem to require phosphorylation 93\#

3.3.1.\# Eps8 from insect cells is quantitatively phosphorylated 93\#

3.3.2.\# Phosphatase treatment of Eps8 has no major impact on its Fbxw5mediated ubiquitylation 93\#

4.\# Nek9 phosphorylates Eps8 and inhibits its Fbxw5-dependent ubiquitylation 96\#

4.1.\# Nek9 interacts with and phosphorylates Eps8 96\#

4.2.\# Nek9 phosphorylation of Eps8 inhibits its Fbxw5-mediated ubiquitylation 98\#

4.3.\# Nek9 might regulate Eps8 levels in vivo 101\#

4.3.1.\# Phosphatase treatment of HeLa cytosol increases the amount of $\begin{array}{ll}\text { co-purifying Eps8 in Nek9 IPs 101\# } & \text { 1Ps }\end{array}$

4.3.2.\# Overexpression of constitutively active Nek9 stabilizes Eps8 levels $\begin{array}{ll}\text { in HeLa cells } & 102 \#\end{array}$

4.4.\# Mass spectrometry analysis reveals Nek9-dependent phosphorylation

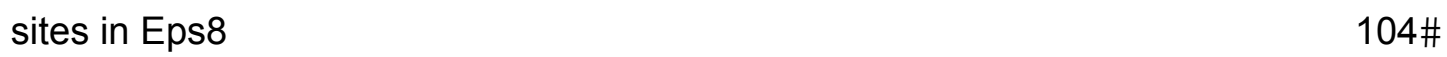

DISCUSSION 107\#

1.\# Fbxw5 - a F-box protein with unconventional features 107\#

1.1.\# Fbxw5 - a bifunctional substrate recognition subunit 107\#

1.2.\# Substrate recognition by Fbxw5 - negative regulation by phosphorylation? $110 \#$

\section{2.\# Eps8 is a target of Fbxw5-dependent ubiquitylation}

- when, how, and why? 114\#

2.1.\# Possible functions of Eps8 ubiquitylation by SCF ${ }^{\mathrm{Fbxw}}$ 115\#

2.2.\# Fbxw5-mediated Eps8 degradation - stimulus- or sub-pool-dependent? 116\#

2.3.\# Nek9 - a putative regulator of Eps8 degradation? 117\#

2.4.\# Identification of Eps8 as a target for Fbxw5 - general roles for Fbxw5
in cell growth or migration?

3.\# Concluding remarks and perspectives 120\#

REFERENCES 122\#

ABBREVIATIONS 134\#

CURRICULUM VITAE 138\# 


\section{LIST OF TABLES}

Table 1: Proteins specifically identified in flag-Fbxw5 immunoprecipitates by mass spectrometry screen.

Table 2: Summary of novel Fbxw5 interaction partners identified by mass spectrometry or western blot analysis.

\section{LIST OF FIGURES}

Figure 1: Basic mechanism Ubiqutiylation.

Figure 2: Ubiquitylation regulates different cellular degradation pathways. $\quad 19$

Figure 3: HECT and RING E3s work by different mechanisms. 21

Figure 4: Cullin-RING Ubiquitin ligases are modular E3 complexes. 23

Figure 5: Model: CAND1/Nedd8 cycle regulates CRL activity. 25

Figure 6: Overall structure of SCF ${ }^{\text {Skp2 }}$. 27

Figure 7: Domain structure of mammalian F-box proteins. 29

Figure 8: Domain structure of Fbxw5 and putative Fbxw5-containing CRL complexes.

Figure 9: Fbxw5 interacts with components of both Cul1- and Cul4-based E3 ligases.

Figure 10: Downregulation of Cul1 stabilizes Fbxw5 levels in HeLa cells. $\quad 75$

Figure 11: Mass Spectrometry analysis of flag-Fbxw5 immunoprecipitates. $\quad 78$

Figure 12: Eps8 and Nek9 but not ACAP2 specifically interact with flag-Fbxw5. 80

Figure 13: flag-Fbxw5 interacts with Nek9, Eps8, TRIM28 and TSC2. 81

Figure 14: Fbxw5 endogenously interacts with Eps8 and Nek9. 83

Figure 15: The interaction between Fbxw5, Nek9 and Eps8 does not require Fbxw5's F-box domain.

Figure 16: Overexpression of Fbxw5 or Fbxw5DF-box does not influence steady state levels of Eps8 in HeLa cells.

Figure 17: Fbxw5 knock down leads to an increase in Eps8 levels.

Figure 18: Eps8 is ubiquitylated by flag-Fbxw5 but not by flag-Fbxw5 $\Delta \mathrm{F}-\mathrm{box}$ immunoprecipitates in vitro.

Figure 19: $\mathrm{SCF}^{\mathrm{Fbxw5}}$ can be reconstituted in vitro.

Figure 20: $\mathrm{SCF}^{\mathrm{Fbxw5}}$ ubiquitylates Eps8 in vitro. 
Figure 21: Eps8 from SF9 cells is quantitatively phosphorylated.

Figure 22: Phosphatase treatment of Eps8 has no major impact on its ubiquitylation by $\mathrm{SCF}^{\mathrm{Fbxw5}}$. 94

Figure 23: Current working model: 95

Figure 24: Eps8 co-purifies with Nek9 from HeLa suspension cells. 97

Figure 25: Nek9 phosphorylates Eps8 in vitro.

Figure 26: Nek9 phosphorylation of Eps8 inhibits its Fbxw5-mediated ubiquitylation.

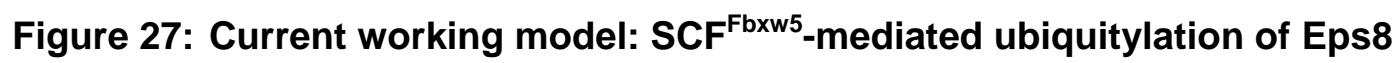
is negatively regulated by Nek9 phosphorylation.

Figure 28: Phosphatase treatment of HeLa cytosol increases the amount of Eps8 in endogenous Nek9 immunoprecipitates.

Figure 29: Overexpression of constitutively active Nek9 stabilizes Eps8 levels in HeLa cells.

Figure 30: Mass spectrometry identification of Nek9-dependent phosphorylation sites within Eps8.

Figure 31: Overview of known and newly identified phosphorylation sites within Eps8.

Figure 32: Models of how Fbxw5 might function as a SRS with two distinct CRL scaffolds.

Figure 33: Phosphodegron-recognition by Fbxw7 and Fbxw1 ( $\beta$-TRCP1) is similar and requires residues at three conserved positions within the WD40 repeat.

Figure 34: Eps8 regulates actin cytoskeleton remodeling and receptor endocytosis. 


\section{ACKNOWLEDGEMENTS}

First of all, I would like to thank my PhD supervisor Prof. Dr. Frauke Melchior for a challenging and interesting project, many long and inspiring discussions, and her guidance, support, and encouragement during my PhD.

I also want to thank the members of my thesis committee Prof. Dr. Reinhard Lührmann and Prof. Dr. Gerhard Braus for valuable advice on my project.

I am also grateful to Dr. Steffen Burkhardt and the Molecular Biology Coordination Office, who did an excellent job in taking care of all my student issues throughout the past five years.

Many thanks go to our collaborators Matthew Calabrese and Brenda Schulman for valuable discussion and reagents concerning SCF complexes, Andrea Disanza, Giorgio Scita, and Per Paolo Di Fiore for important advice and reagents concerning Eps8, and Henning Urlaub and his lab for doing a great job in mass spec matters.

I want to thank the DFG for funding my PhD project in conjunction of the SPP1365.

Further thanks go to Ulrike G. for excellent technical and intellectual support in the last year of my $\mathrm{PhD}$, as well as to Janina and Anette for critically reviewing my PhD Thesis and to Janina again for her help with purifying Fbxw5 from SF9 cells.

I want to thank all former and current members of the Melchior lab for excellent discussions, for a great working atmosphere, and for all the fun times we had on retreats, RUBICON conferences, and when simply sitting together having beer or cake. Special thanks go to Marie-Christine, Tina, and Anette for many "Bier ab vier" and to Ruth, Sarah S., Erik, and Ulrike M. formerly known as the "small lab". 
I am also very grateful for the great times I had with my friends from Göttingen, especially those I got to know via the IMPRS Molecular Biology and Neuroscience. Thank you for a great time during the past years!!!

Abschließend danke ich den Menschen, denen ich am meisten zu verdanken habe: meinen Eltern und meiner Schwester, die mich immer und bedingungslos unterstützt haben. 


\section{LIST OF PUBLICATIONS}

Werner A, Becker J, Calabrese M, Disanza A, Gern U, Urlaub H, Scita G, Schulman B, Melchior F (2010) Eps8 ubiquitylation by SCF ${ }^{\mathrm{Fbxw} 5}$. (manuscript in preparation)

Blume A, Werner A, Koehli T, Wakarchuk WW, Ernst B, Peters T (2010) Fragment-based screening of substrate specificity of the sialyltransferase Cstll from Camphylobacter jenjuni using STD-NMR. (manuscript in preparation)

Bethani I, Werner A, Kadian C, Geumann U, Jahn R, Rizzoli SO (2009) Endosomal fusion upon SNARE knockdown is maintained by residual SNARE activity and enhanced docking. Traffic 10:1543-1559 


\section{ABSTRACT}

Targeted protein ubiquitylation that occurs in eukaroytes is often performed by cullin-RING-based E3 Ubiquitin ligases (CRLs), a superfamily of E3s. These types of ligases are composed of a modular E3 core containing a cullin and a RING domain protein, and a substrate specificity module usually composed of a linker protein and a substrate recognition subunit (SRS). The combination of distinct substrate specificity modules with core components creates unique CRL complexes that bind distinct sets of substrates.

The rather uncharacterized F-box and WD40 repeats containing protein 5 (Fbxw5) represents a special SRS, since it has the potential to assemble into two different types of CRLs: via its F-box domain into Cul1-based (SCF) complexes and via DWD boxes into Cul4A-based complexes. Up to date, it has remained elusive, whether Fbxw5 assembles into functional SCF complexes in vivo.

Here, we show that in mammalian tissue culture cells under normal growth conditions, overexpressed and endogenous Fbxw5 predominantly assembles into SCF complexes and we identify the epidermal growth factor kinase substrate 8 (Eps8) as the first target of SCF ${ }^{\mathrm{Fbxw} 5}$. Eps8 is a key regulator of actin cytoskeleton remodeling and receptor endocytosis that - when upregulated - can contribute to increased migration and invasiveness of cancer cells. Fbxw5 interacts with Eps8 endogenously, reconstituted $\mathrm{SCF}^{\mathrm{Fbxw5}}$ ubiquitylates Eps8 in vitro, and downregulation of Fbxw5 in HeLa cells can result in stabilization of Eps8, indicating that SCF ${ }^{\mathrm{Fbxw5}}$ targets Eps8 for degradation in vivo. Moreover, Nek9, a kinase recently implicated as a regulator of autophagy, endogenously co-purifies with Fbxw5 and Eps8 from cells and phosphorylates Eps8 in vitro at several different sites. Interestingly, these phosphorylations inhibit $\mathrm{SCF}^{\mathrm{Fbxw} 5}$-mediated ubiquitylation of Eps8 in vitro and overexpression of constitutively active Nek9 stabilizes Eps8 levels in HeLa cells.

Taken together, these findings provide evidence that Fbxw5 can function in the context of two distinct CRL complexes, identify Fbxw5 as a protein to regulate Eps8 levels in cells, and suggest a rather unexpected regulatory role of phosphorylation in Fbxw5-mediated Eps8 ubiquitylation. 


\section{INTRODUCTION}

A common feature of all living organisms is their employment of proteins to build molecular machineries that take on the complex network of cellular activities. The abundance of a certain protein element is spatially and temporally regulated in two counteracting ways: de novo synthesis to produce sufficient amounts of the protein, and degradation when it is in excess, damaged, or no longer functionally required. One key player in coordinating different routes of protein destruction within cells is the Ubiquitin conjugation system, which marks target proteins for proteasomal or lysosomal degradation in a tightly regulated and highly specific manner.

\section{Ubiquitin and its conjugation to proteins}

Ubiquitin is a 76-residue polypeptide that is highly conserved among eukaryotes. It is the founding member of a class of structurally related peptide modifiers that exert influence on an enormous range of physiological processes (Pickart and Eddins 2004; Hochstrasser 2009). The conjugation of Ubiquitin to substrates termed ubiquitylation - usually involves three enzymatic steps (Hershko and Ciechanover 1998) (Figure 1): initially, Ubiquitin is activated by an Ubiquitin E1 enzyme in an ATP-dependent manner. Subsequently, Ubiquitin is transferred to one of several E2 enzymes (Ubiquitin conjugating enzymes - Ubcs). In the final step, Ubiquitin is conjugated to its substrate by one of hundreds of E3 enzymes that contribute to substrate specificity (Pickart 2001). The human genome encodes two E1 enzymes, 37 E2 enzymes and more than 600 E3 ligases (Groettrup et al. 2008; Deshaies and Joazeiro 2009; Markson et al. 2009). Ubiquitylation is a dynamic process and can be readily reversed by a superfamily of approximately 85 isopeptidases termed deubiquitinases (DUBs, recently reviewed in (Komander, Clague, and Urbe 2009)). 


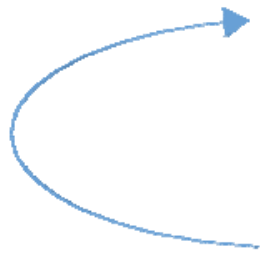

DUBs (deubiquitinases)

(f)

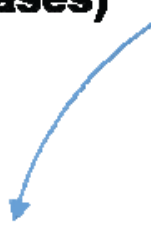

Ubi

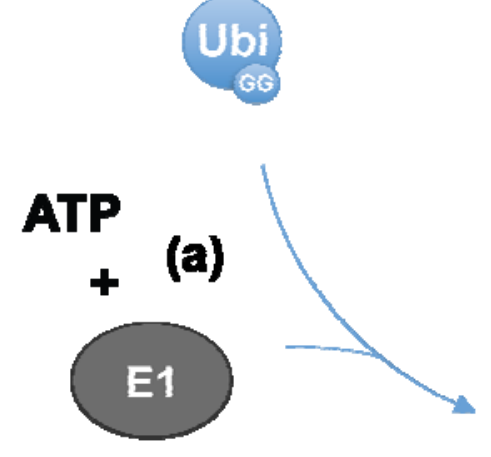

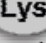

Target
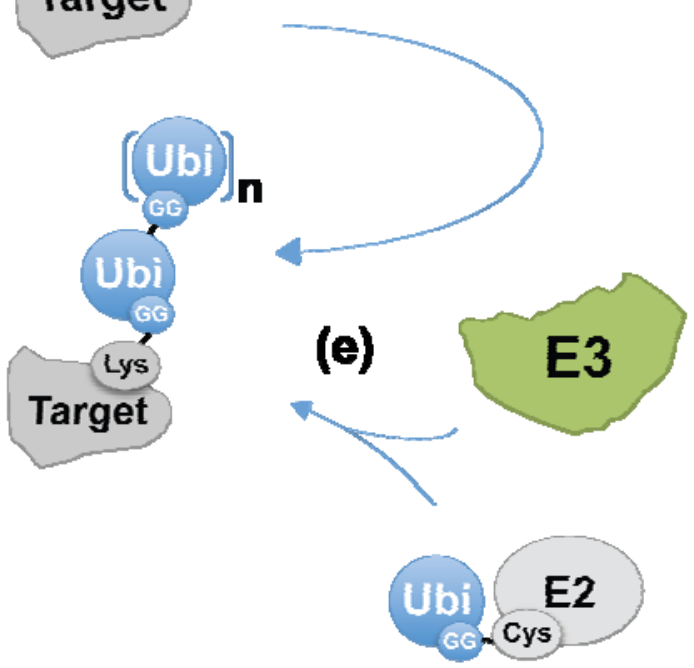

(d)

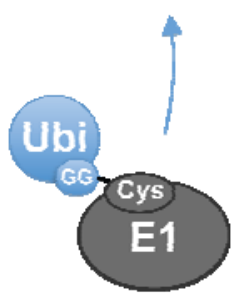

(c)

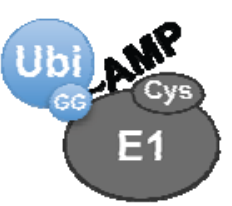

(b)

Figure 1: Basic mechanism Ubiqutiylation. Mature Ubiquitin is activated and attached to target proteins by three types of enzymes. In a first ATP-consuming step (a), an E1 enzyme activates Ubiquitin by adenylating the carboxyl-group of the C-terminal glycine residue of Ubiquitin. The Ubiquitin-AMP adduct remains bound to the E1 enzyme (b). Then, the catalytic cysteine residue of the E1 attacks the C-terminus of Ubiquitin, yielding an E1-Ubiquitin-thioester (c). In a transthiolation reaction, one of several E2 enzymes picks up the Ubiquitin (d). With the assistance of one of several hundreds of E3 ligases, the E2 enzyme then transfers the Ubiquitin to the target, typically forming an isopeptide bond between the C-terminal carboxyl-group of Ubiquitin and the $\varepsilon$ amino-group of a lysine residue within the target (e). In this process, the E3 ligases are crucial for substrate recognition (for more details see introduction section 4, page 20). For Ubiquitin chain formation, the N-terminal amino-group or lysine side chain of one Ubiquitin molecule is linked to the C-terminus of another Ubiquitin molecule. Ubiquitin modification is only transient since enzymes known as deubiquitinases (DUBs) can remove Ubiquitin molecules that are attached to proteins (f). 


\section{Forms of ubiquitylation}

Target proteins can be either modified by a single Ubiquitin molecule (monoubiquitylation), several single Ubiquitin moieties (multi-ubiquitylation) or by Ubiquitin chains (poly-ubiquitylation). These Ubiquitin chains are formed through conjugation of Ubiquitin to a preceding Ubiquitin moiety, and can take on diverse structures and functions, depending on their linkage (most recently reviewed in (Komander 2009)). All lysine residues (Lys6, Lys11, Lys27, Lys29, Lys33, Lys48, Lys63) as well as the N-terminal amino group of Met1 within Ubiquitin are used for chain formation in vivo (Kirkpatrick et al. 2006; Tokunaga et al. 2009). In S. cerevisiae, quantative proteomics has revealed that the most abundant lysine linkages are Lys48 and Lys11, followed by Lys63, Lys6, and Lys27 (Xu et al. 2009).

\section{Functional outcomes of ubiquitylation}

The functional outcome of Ubiquitin conjugation to a substrate depends, in large part, on the type of Ubiquitin modification. Extensive structural, biochemical, and bioinformatical studies over the last decade have helped to get an initial understanding of how these different forms of ubiquitylation can result in distinct physiological outcomes (reviewed in (Hurley, Lee, and Prag 2006; Komander 2009)). Mono-ubiquitylation creates a new binding surface on the target that allows recognition by proteins that contain at least one of approximately 20 specialized Ubiquitin binding domains (UBDs) (Dikic, Wakatsuki, and Walters 2009). These domains commonly recognize a prominent hydrophobic surface patch centered on Ile44 in Ubiquitin. In addition, differently linked poly-Ubiquitin chains have diverse structures that allow discrimination by deubiquitinases and UBD-containing proteins. This way, different forms of ubiquitylation can be translated into distinct signals, providing an explanation of how ubiquitylation is able to simultaneously participate in several degradation pathways and a wide variety of non-proteolytic processes within the cell. 


\subsection{Degradation functions of Ubiquitin}

\subsubsection{Proteasome-dependent degradation}

The first identified and probably the best-characterized function of ubiquitylation is tagging proteins for degradation by the $26 \mathrm{~S}$ proteasome (Ciechanover et al. 1980; Glickman and Ciechanover 2002). In this process (Figure 2a, recently reviewed in (Finley 2009)), poly-ubiquitylated proteins are targeted to the regulatory subunit of the proteasome via recognition by one of five currently known proteasomeassociated, UBD-containing Ubiquitin receptors. Either the poly-ubiquitylated proteins are directly bound by the integral proteasome subunits, Rpn10 and Rpn13, or they are recognized by so called "shuttle factors" (Ddi1, Dsk2, or Rad23) that capture substrates somewhere within the cell and escort them to the proteasome. Once targeted to the regulatory subunit, substrates are unfolded by a ring of six AAA ATPases and deubiquitylated so that they can be translocated through a narrow gated channel into a chamber within the 28-subunit proteolytic core particle, where they are hydrolyzed to peptides.

For many years, the canonical view was that Ubiquitin modification only in form of a chain of at least four Lys48-linked Ubiquitins (and in some cases also Lys29linked polymers) function as a general device for targeting proteins for proteolysis by the proteasome (Pickart 2000). Recent studies have led to a more complex picture: also K11-linked chains and even K63-linked chains, which have been found in many instances to provide a signaling rather than degradation function (see below, section 3.2), have been demonstrated to target substrates to the proteasome in vivo (Jin et al. 2008; Saeki et al. 2009; Xu et al. 2009).

\subsubsection{Lysosome-dependent degradation}

Next to targeting proteins to the proteasome, ubiquitylation has also been implicated in mediating the lysosomal degradation of membrane proteins via the ESCRT pathway (Figure 2b). Recent findings suggest the involvement of ubiquitiylation in yet another fundamental lysosome-dependent degradation system, namely autophagy (Figure 2c). 
Lysosomal degradation of membrane proteins

Mono-, multi-, or short K63-linked poly-ubiquitylation have been shown to be required for plasma membrane internalization and lysosomal targeting of activated growth factor receptors in mammals, as well as various transporters and permeases in yeast (reviewed in (Welchman, Gordon, and Mayer 2005)). Ubiquitylation of these membrane proteins promotes their sorting into luminal vesicles of late endosomes or multivesicular bodies, thereby committing them for degradation by lysosomes (Figure $2 \mathrm{~b}$ ). This sorting process from the early endosome to the intralumenal vesicles of late endosomes is mediated by the successive action of at least four different multiprotein complexes known as endosomal sorting complexes required for transport (ESCRTs), which can each recognize ubiquitylated cargo through UBDs (Wollert et al. 2009).

\section{Lysosomal degradation via selective autophagy}

Selective autophagy is the most recently discovered degradation process ubiquitylation has been linked to. The general term autophagy refers to processes by which cytoplasmic materials reach lysosomes for degradation (Kroemer and Levine 2008). Among three types of autophagy, macroautophagy is the most extensively studied. During macroautophagy a small vesicular sac, termed phagophore, elongates and subsequently encloses a portion of cytoplasm to generate a double-membraned structure called autophagosome. The autophagosome can fuse with lysosomes, leading to the formation of autophagolysosomes, in which the enclosed materials are degraded. Amongst other crucial components, the formation of autophagosomes requires an autophagy-specific Ubiquitin-like conjugation systems that covalently couples LC3/GABARAP to phosphatidylethanolamine on the phagophore membrane (reviewed in (He and Klionsky 2009)). While macroautophagy has long been regarded as a random cytoplasmic degradation system, a series of recent publications (reviewed in (Kirkin et al. 2009; Korolchuk, Menzies, and Rubinsztein 2010)) have changed this view. At least in special cases, Ubiquitin seems to serve as a selective signal for targeting various cellular cargos ranging from individual cytosolic proteins and large macromolecular complexes to organelles for degradation by autophagy. In this process, autophagy receptors, such as p62 
and NBR1, which simultaneously bind Ubiquitin and LC3/GABARAP, mediate the engulfment of ubiquitylated substrates into autophagosomes (Figure 2c). Upon lysosomal fusion, these targets are degraded by acidic hydrolases. At a molecular level, this process is rather ill defined; neither the type of ubiquitylation has been unambiguously identified, nor the question has been answered, whether deubiquitylation is required before engulfment of the substrates.

\subsection{Non-degradation functions of Ubiquitin}

A great variety of regulatory functions of Ubiquitin beyond protein degradation have been uncovered at a very rapid pace over the last decades. Hence, ubiquitylation is nowadays - similar to protein phosphorylation - viewed as a fundamental regulatory post-translational signaling event (Woelk et al. 2007).

So far, most non-degradation functions of ubiquitylation have been attributed to mono-ubiqutiylation and Lys63-linked Ubiquitin chains. For example, both types of ubiquitylation have been shown to trigger endocytosis of membrane receptors (Strous and Govers 1999; Hicke and Dunn 2003); in addition they have been shown to be intimately involved in DNA-damage response by modification of the DNA sliding clamp PCNA, thereby determining how cells replicate after damaged DNA (reviewed in (Moldovan, Pfander, and Jentsch 2007)). Further functions of mono-ubiquitylation involve transcriptional regulation (Conaway, Brower, and Conaway 2002), and chromosomal silencing (Sun and Allis 2002), whereas Lys63-linked chains have been shown to regulate ribosomal function (Spence et al. 2000) and cytokine signaling by activation of the TAK1 and IKK protein kinase complexes (reviewed in (Skaug, Jiang, and Chen 2009)).

Most other types of ubiquitylation, e.g. chains involving links other than Lys48 and Lys63, have just recently started to attract researcher's notice. For example, modification of the IKK complex component NEMO with linear Ubiquitin chains was recently found to be required for NF-кB activation (Tokunaga et al. 2009).

Given that some other chain types have hardly been studied so far, it is to be expected that the manifold spectrum of functions of ubiquitylation will expand even further in the future. 


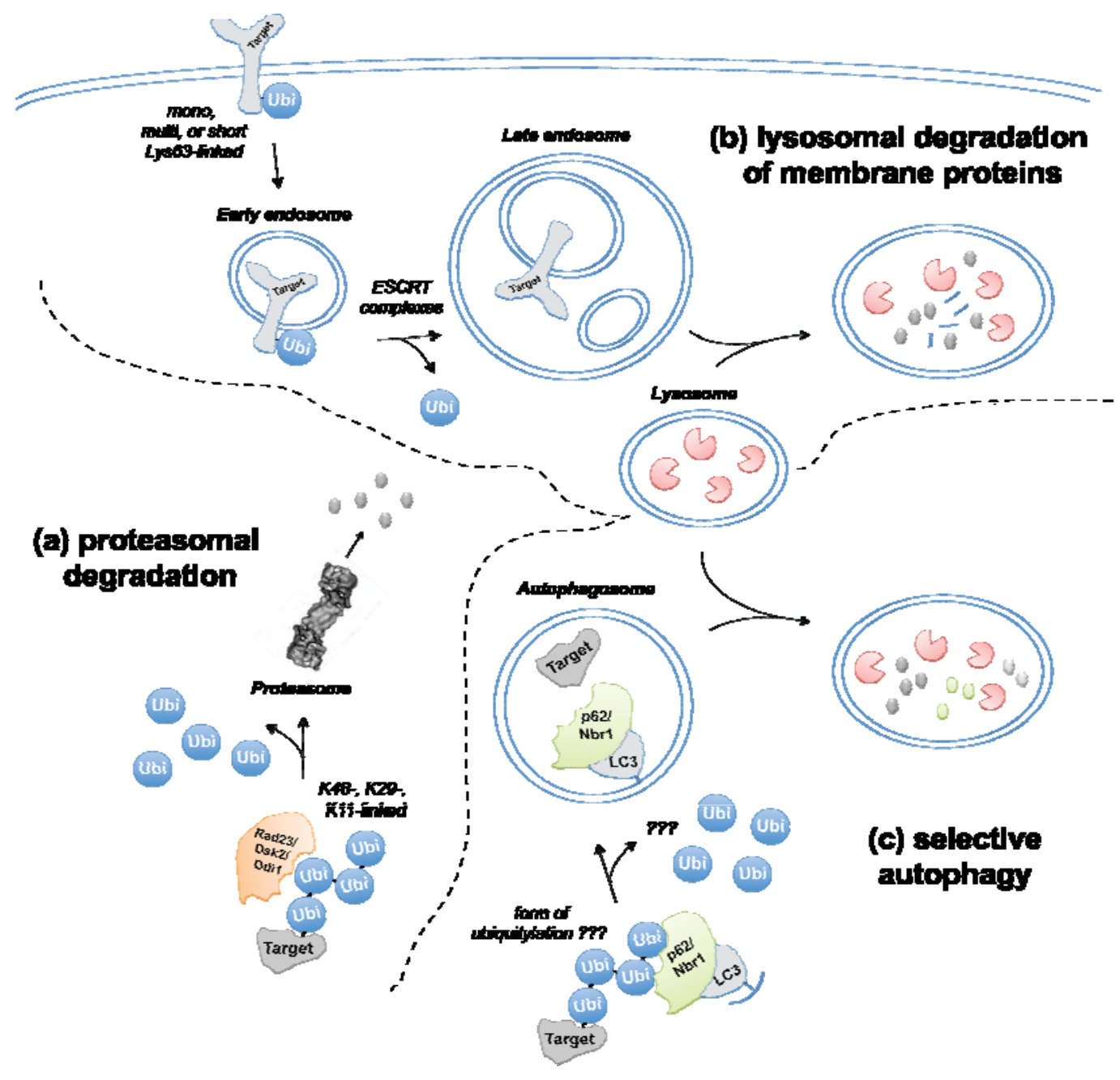

Figure 2: Ubiquitylation regulates different cellular degradation pathways. (a) Proteasomal degradation: Modification with Lys48-, Lys29-, Lys11-linked (and maybe also differently linked) Ubiquitin chains mediates the delivery of targets to the $26 \mathrm{~S}$ proteasome. The Ubiquitin chain is either recognized by Ubiquitin receptors that are integral parts of the proteasome (not shown) or shuttling factors (depicted in orange) that deliver substrates to the proteasome. Substrates are unfolded and deubiquitylated at the regulatory subunit of the proteasome prior to entry in the proteolytic core subunit, where hydrolysis into peptides occurs. (b) Lysosomal degradation of membrane proteins: Mono- and multi-ubiquitylation (and in some cases also short Lys63-linked Ubiquitin chains - not shown) mediate both endocytosis and sorting of membrane proteins (e.g. growth factor receptors) into late endosomes / multivesicular bodies for lysosomal degradation. The sorting steps is controlled by the action of so called ESCRT complexes, which directly recognize the Ubiquitin moieties and orchestrate the targeting of the substrates into intralumenal vesicles of the late endosome. Upon fusion of late endosomes with lysosomes, targets are degraded by acidic hydrolases (depicted in red). Ubiquitin itself is not targeted for lysosomal degradation within this process, but rather recycled by deubiquitylation. (c) Lysosomal degradation via selective autophagy: The autophagy receptors p62 and Nbr1 (depicted in green) can bind both, the autophagosome-specific LC3/GABARAP modifiers (via an LC3interacting (LIR-) motif) and ubiquitylated proteins (via an UBD). This way, they are thought to mediate the engulfment of selective substrate proteins (marked by ubiquitylation) into autophagosomes. The molecular details of this process (e.g. type of ubiquitylation, fate of Ubiquitin etc.) have not been clarified so far. 


\section{Regulating target specificity of ubiquitylation - E3 ligases}

The plethora of Ubiquitin's biological targets and functions raises the question how specificity is ensured within the ubiquitylation pathway. In large part, this task is taken on by a huge superfamily of enzymes known as Ubiquitin E3 ligases. These proteins confer specificity to ubiquitylation by recognizing target substrates and mediating transfer of Ubiquitin from an E2 enzyme to a specific substrate.

\subsection{Types of Ubiquitin E3 ligases}

There are two major types of E3 ligases in eukaryotes that are distinguished by their domain used to recognize the E2 enzyme and their biochemical way of

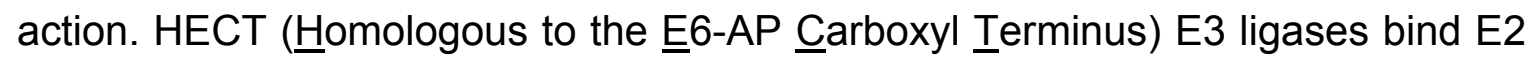
enzymes via a HECT domain, which contains a conserved Cys residue that forms an intermediate thioester bond with the Ubiquitin C-terminus before catalyzing substrate ubiquitylation (Figure $3 \mathrm{~A}$ ). In contrast, RING (Really interesting new gene) E3 ligases contain an E2-recognizing RING domain and act as scaffolds that bring the E2 near the substrates to facilitate the transfer of Ubiquitin to the substrate (Figure 3B). In addition, there is a third, rather small family of E3s that are defined by an E2-binding domain called U-box. This domain is structurally related to the RING domain and the mechanism of catalysis of U-box-containing E3s is also similar to that of RING E3s (Pickart and Eddins 2004).

With hundreds of different targets for ubiquitylation, there is also the requirement for a large number of Ubiquitin E3 ligases. Indeed, there are more than 600 distinct E3 ligases in humans (Deshaies and Joazeiro 2009). Generation of this large number of E3s from just three principle protein domains for E2 recruitment is made possible by the modular construction of E3 enzymes. In a particular E3, the E2-binding domain is spatially separated from domain(s) dedicated to interact with the substrates of that E3 (see Figure 3). These two types of domains can be part of the same polypeptide (as is generally the case for HECT E3s (Rotin and Kumar 2009) and many RING E3s (Deshaies and Joazeiro 2009)) or they can belong to different subunits of a multi-protein complex, as in the case of the RING domain proteins Apc11 (Matyskiela, Rodrigo-Brenni, and Morgan 2009) and 
$\mathrm{Rbx} 1 / 2$. In particular, $\mathrm{Rbx} 1 / 2$ adds great diversity to the $\mathrm{E} 3$ family by forming Skp1-Cul1-F-box (SCF) and other Cullin-RING Ubiquitin ligase (CRL) complexes (Petroski and Deshaies 2005).

A)

B)

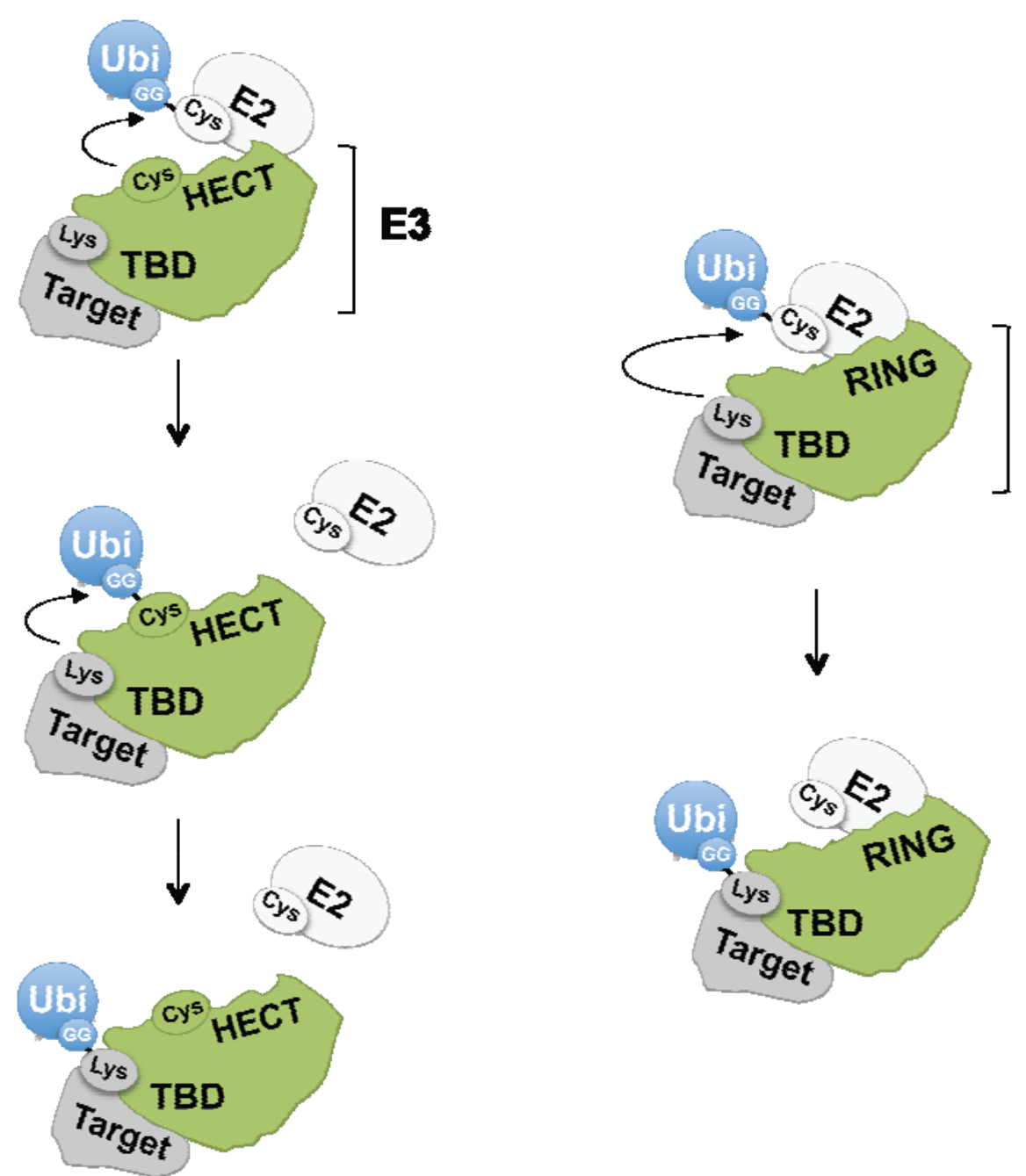

E3

Figure 3: HECT and RING E3s work by different mechanisms. A) HECT E3s have a conserved cysteine residue that accepts Ubiquitin from the E2 enzyme (bound to the HECT domain) to form an E3-Ubiquitin thioester. Ubiquitin is then transferred from this covalent E3 intermediate to the substrate (bound by a target binding domain - TBD). B) By contrast, RING E3s facilitate the direct transfer of Ubiquitin from the E2 to the target. 


\subsection{Cullin-RING based Ubiquitin E3 ligases (CRLs)}

\subsubsection{Architecture of CRL complexes}

Cullin-RING complexes are the largest known class of Ubiquitin E3 ligases. They regulate diverse cellular processes, including multiple aspects of the cell cycle, transcription, signal transduction, and development (Petroski and Deshaies 2005). Human cells express seven different cullin proteins (Cul1, 2, 3, 4A, 4B, 5, and 7) that each nucleate a multi-subunit RING E3 ligase (see Figure 4).

In these cullin-ㅍN as a scaffold to connect the E2-binding module $(\mathrm{Rbx} 1 / 2)$ with the substrate recognition subunit (SRS). With the exception of Cul3-based complexes (Pintard, Willems, and Peter 2004), the interaction of the SRS with cullin is not direct but bridged by an adaptor protein. While the cullin, $\mathrm{Rbx} 1 / 2$, and the adaptor protein form the constitutive core, the SRS is interchangeable. One exception might be CRL7, in which Cul7 has only been shown to interact with Fbxw8 as SRS so far (Dias et al. 2002). Usually, the SRS contains one domain for substrate recognition and a domain for interaction with one particular CRL complex. SRSs that contain an F-box assemble into SCF/CRL1 complexes, those SRSs with a VHL-Box assemble into CRL2 complexes, those with a BTB domain into CRL3 complexes, those with DWD-boxes into CRL4 complexes, and those with a SOCS-Box into CRL5 complexes. With more than 300 human genes encoding for proteins with such domains, a great variety of different CRLs with a broad range of substrate specificity can potentially be formed within cells (Deshaies and Joazeiro 2009).

\subsubsection{General mechanisms of CRL complex regulation}

Whereas the diversity of substrate recognition subunits (SRS) of cullin-ㅁING based Ubiquitin E3 ligases (CRLs) specifies which substrates are targeted for ubiquitylation, the activity of the catalytic core is further modulated by several regulatory mechanisms, which are thought to be similar for the different types of CRLs. 


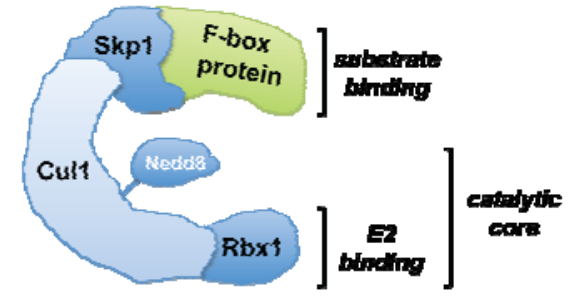

CRL1 (SCF)

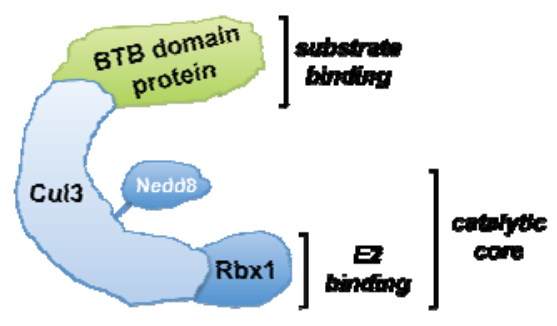

CRL3

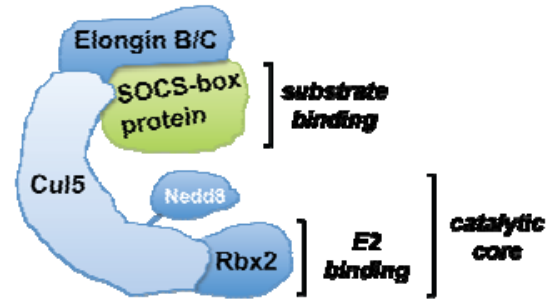

CRL5

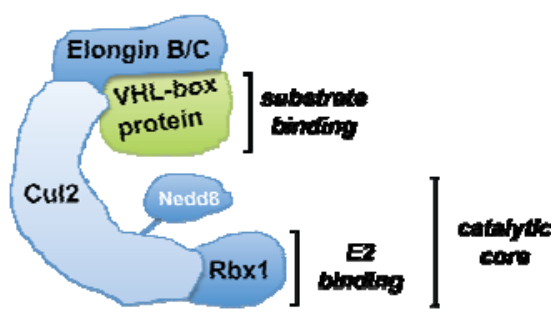

CRL2

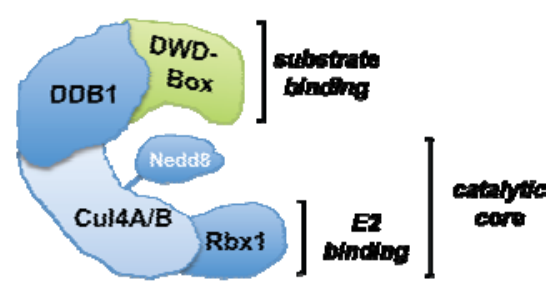

CRL4

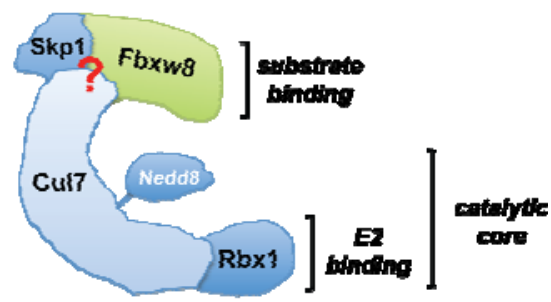

CRL7

Figure 4: Cullin-RING Ubiquitin ligases are modular E3 complexes. Human Cullin-RING E3 ligases are built from one of seven cullin proteins (Cul1, 2, 3, 4A, 4B, 5, and 7), an E2-binding RING domain protein, a substrate binding subunit (SRS) and usually an adaptor protein. The common catalytic core of CRLs is made up of the C-terminal part of the cullin protein that interacts with the RING protein Rbx1/2. N-terminally each cullin protein recognizes its specific adaptor protein that links the SRS to the complex (with the exception of Cul3 that directly interacts with the SRS). Generally, the SRSs are interchangeable, which allows for formation of different CRLs of one class with distinct substrate specificities. CRL1s, which are commonly known as Skp1-Cul1F-box (SCF) complexes, recruit substrates through the adaptor protein Skp1 and an F-box protein substrate receptor. CRL2s and CRL5s recruit substrates through an elongin-BC adaptor and an SRS that contains a Von-Hippel-Lindau (VHL)-box or a suppressor of cytokine signaling (SOCS)box, respectively. CRL3s recruit substrates through 'Broad Complex, Tramtrack, Bric-a-Brac' (BTB)-domain-containing substrate receptors. CRL4s recruit substrates via the adaptor protein DNA-damage-binding protein 1 (DDB1) and SRSs that contain a DWD-box. CRL7s seem to recruit substrates only via one SRS: Fbxw8. Whether the interaction between Cul7 and Fbxw8 is direct or dependent on Skp1 is currently unclear. All cullin proteins can be neddylated at a conserved lysine residue in the catalytic core. Neddylation has been shown to be required for full catalytic activity of these enzyme complexes (see section 4.2.2). 
Nedd8 attachment promotes CRL activity

All cullins identified so far can be modified by covalent attachment of the small Ubiquitin-like modifier Nedd8 at a conserved lysine residue in the cullin homology domain (Hori et al. 1999; Osaka et al. 2000). This process, termed neddylation, is mechanistically similar to the ubiquitylation reaction but involves different sets of enzymes (Rabut and Peter 2008). Nedd8 conjugation increases Cul1-based Ubiquitin E3 ligase activity in vitro and is required for the in vivo function of Cul1, Cul2, and Cul3 (Petroski and Deshaies 2005). Detailed biochemical and structural work comparing unneddylated with neddylated CRLs demonstrated that Nedd8 promotes $C R L$ activity through conformational changes of the catalytic core, thereby stimulating E2 recruitment, substrate ubiquitylation, and chain elongation (Duda et al. 2008; Saha and Deshaies 2008).

Reversible cycles of neddylation and deneddylation and CAND1 seem to regulate $C R L$ subunit stability and CRL assembly and disassembly

Nedd8 is removed from cullins in a process, called deneddylation, by the isopeptidase activity of the metallo-protease CSN5 of the COP9 signalosome (CSN) (Cope et al. 2002). This process is thought to be crucial for CRL activity in two ways: first of all, by reducing CRL activity when not required to prevent autoubiquitylation and degradation of SRSs (Cope and Deshaies 2006). Secondly, deneddylation has been shown to trigger disassembly of CRL complexes by working in conjunction with the cullin-associated and neddylationdissociated protein-1 (CAND1). A large number of biochemical and genetic studies over the last years have contributed to a model in which CAND1, neddylation, and deneddylation sustain $C R L$ activity by promoting cycles of CRL assembly and disassembly as depicted in Figure 5 (Cope and Deshaies 2003). 


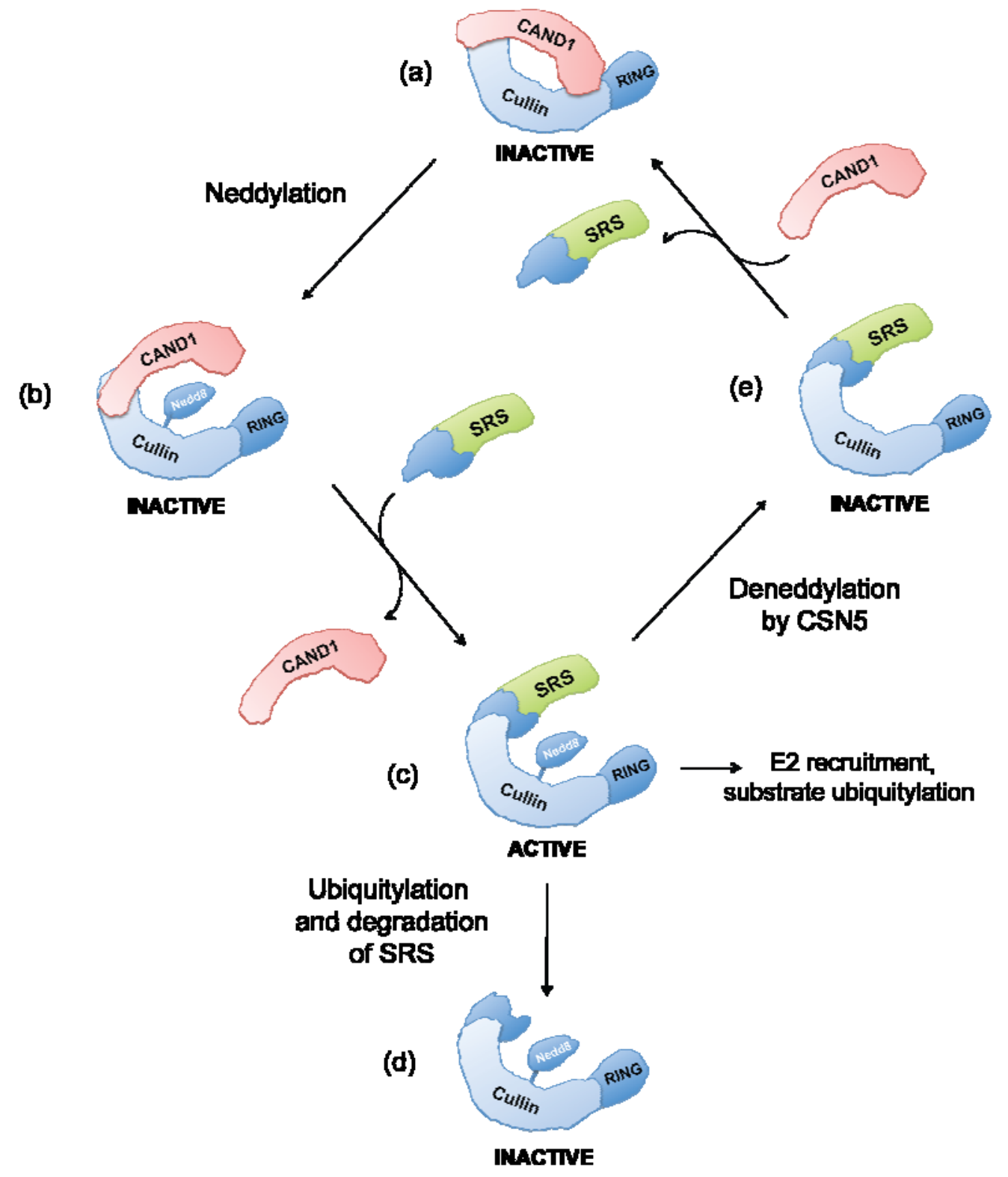

Figure 5: Model: CAND1/Nedd8 cycle regulates CRL activity. When the catalytic core of the CRL is assembled with CAND1, it is held in an inactive state (a). Upon neddylation of the cullin subunit, the cullin-CAND1 interaction is weakened (b) and an incoming adaptor-SRS complex can displace the CAND1 from the cullin to yield an active CRL complex that can ubiquitylate bound substrates (c). In the absence of substrates, the SRS is ubiquitylated and degraded and another SRS might enter the complex (d). Alternatively, Nedd8 might be cleaved from the cullin by the CSN, which enables CAND1 to bind again to the cullin and eventually strip away the adaptor-SRS complex thereby sequestering the cullin (e). Several steps within this model have not been formally demonstrated in vivo and further studies are required to validate and expand this model in the future. 


\subsubsection{Skp1-Cul1-F-box (SCF) Ubiquitin E3 ligases}

\section{Structure of SCF complexes}

The structurally and biochemically best characterized mammalian $C R L$ is the Skp1-Cul1-F-box (SCF) Ubiquitin E3 ligase. As revealed by the crystal structure

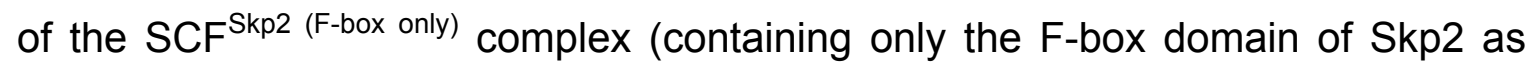
SRS, Figure 6), the overall structure of the complex is highly elongated with the E2-recruiting subunit, Rbx1, and the adaptor-SRS complex, Skp1-Skp2, isolated to opposite ends (Zheng et al. 2002). Cul1 serves as a scaffold by directly interacting with all three subunits: with its $\mathrm{N}$-terminal helical domain that adopts a long tube-like structure, Cul1 interacts with the Skp1-F-box ${ }^{\text {Skp2 }}$ and via its globular C-terminal domain it binds Rbx1. While Cul1 shares an extensive interaction surface with Skp1, the Cul1-Skp2 interaction involves only two residues of the Fbox domain of Skp2 (Pro113 and Glu115) that are conserved amongst most but not all F-box proteins. At least in S. cerevisiae, the proline residue is of functional significance, since it seems to be required for the efficient integration of the Skp1F-box module into SCF complexes (Schmidt et al. 2009).

\section{E2s used by SCF complexes}

Mammalian SCF complexes (and also other mammalian CRLs) have been shown to work with the Ubiquitin E2 enzymes $\mathrm{Cdc} 34$ and $\mathrm{UbcH} 5$ in vitro and in vivo, but the basis of differentiating between these two members of different E2 classes is not yet completely understood (Butz et al. 2005; Wu, Kovacev, and Pan 2010). While Cdc34 readily synthesizes di-Ubiquitin and preferentially poly-ubiquitylates substrates with Lys48-linked chains regardless of the E3 partner, UbcH5 has low activity in di-Ubiquitin formation, preferentially multi-ubiquitylates targets, and forms various types of Ubiquitin chains depending on the E3 partner (Deshaies and Joazeiro 2009). Together with $\mathrm{SCF}^{\beta-T R C P}$ as E3, UbcH5c is fast and efficient in conjugating the first Ubiquitin moiety to various substrates, while it works relatively slow and inefficient in chain elongation. Conversely, Cdc34 attaches the first Ubiquitin relatively slow to targets but efficiently elongates the Ubiquitin chain (Saha and Deshaies 2008). Recent findings suggest that at least in the case of $\mathrm{SCF}^{\beta-\mathrm{TRCP}}$-mediated ubiquitylation of $\mathrm{I}_{\kappa} \mathrm{B} \alpha, \mathrm{Cdc} 34$ and $\mathrm{UbcH} 5 \mathrm{c}$ can cooperate in 
poly-ubiquitylation via a "priming and extension" mechanism (Wu, Kovacev, and Pan 2010).

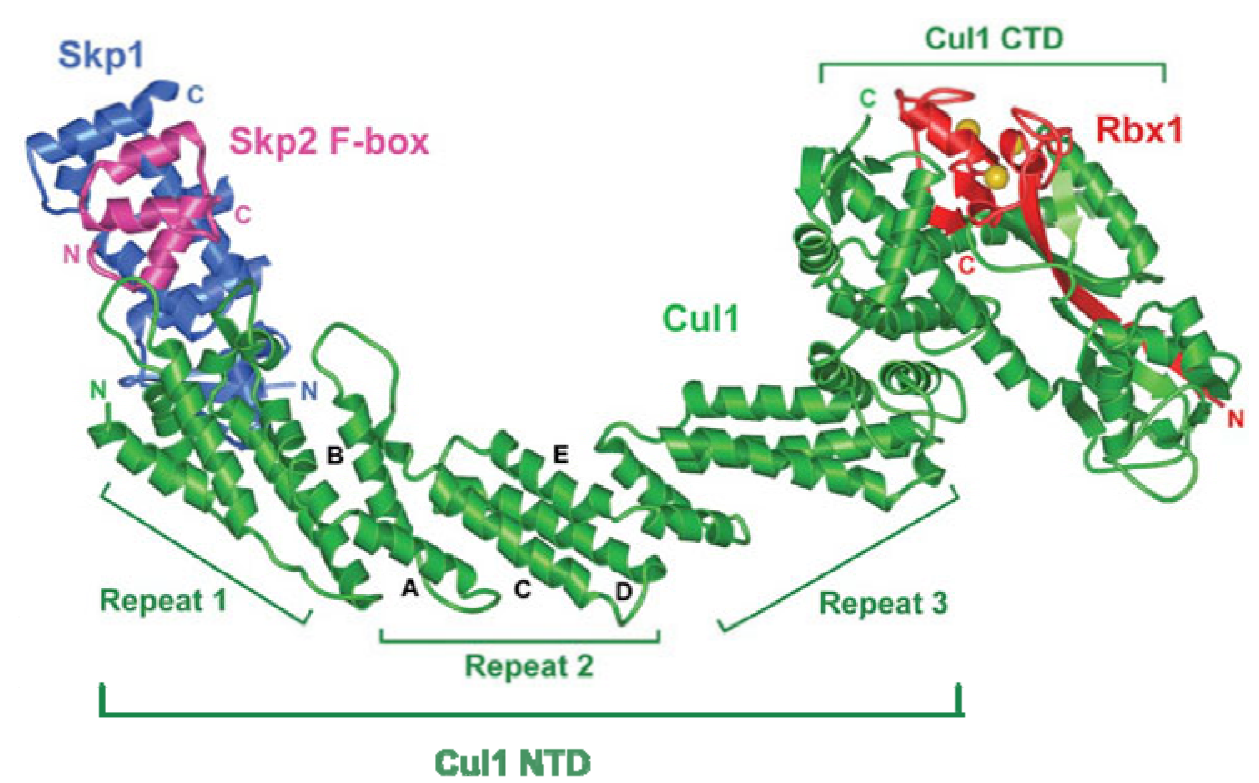

Figure 6: Overall structure of $\mathrm{SCF}^{\text {Skp2 }}$. Cul1, Rbx1, Skp1, and the F-box domain of Skp2 are colored in green, red, blue and magenta, respectively. The picture was taken from (Zheng et al. 2002) and was modified. NTD $=\mathrm{N}$-terminal domain; $\mathrm{CTD}=\mathrm{C}$-terminal domain.

\section{Biological functions of SCF complexes}

Ubiquitylation mediated by SCF complexes (and also by other CRLs) is generally thought to promote the proteasomal degradation of the substrate in question. By targeting key components of different cellular pathways (e.g. signaling adaptors, cell cycle regulators, and transcription factors etc.), SCF complexes contribute to the regulation of a vast variety of biological processes (Cardozo and Pagano 2004). Which precise physiological function a particular SCF complex fulfills, is determined by its substrate specificity and therefore ultimately by the F-box protein it contains. 


\section{F-box proteins}

\subsection{Classification of F-box proteins}

F-box proteins are the variable substrate recognition subunits (SRSs) of SCF E3s. This family of proteins comprises 69 members in humans (Skaar et al. 2009) and is characterized by the presence of a conserved 40 amino acid region that folds into an F-box domain, which mediates binding to Skp1 and thus to the rest of the SCF complex (see Figure 6). F-box proteins are divided into three subfamilies, depending on their homology domains: Fbxw proteins contain WD40 repeats, Fbxl proteins contain leucine-rich repeats and possibly other domains, and Fbxo proteins contain neither WD40 nor leucine-rich repeats, but other variable domains (see Figure 7).

\subsection{Target recognition by F-box proteins}

Most characterized F-box proteins of the Fbxw and Fbxl class bind their substrates through the WD40 and leucine-rich repeats, respectively. This recognition generally requires a post-translational modification of the substrate most often phosphorylation. So far all characterized Fbxw proteins are thought to recognize their targets upon phosphorylation of the substrate within a short amino acid degradation sequence termed degron (Skaar et al. 2009). Prime examples for this are Fbxw1 ( $\beta$-TRCP) and Fbxw7, which recognize phosphorylation(s) within the degron of their respective targets in a highly specific manner via conserved residues within their WD40 repeat regions (Wu et al. 2003; Hao et al. 2007). This way, even in the presence of a F-box protein, a substrate is not recognized by the SCF complex without activation of the proper kinase.

In some cases, F-box proteins can also bind to small accessory proteins (or also small molecules in plants) that play key roles in substrate recognition. For example, the small accessory protein Csk1 is essential for Skp2-mediated ubiquitylation of p27 (Hao et al. 2005) and the plant hormone auxin is required for interactions between the F-box protein TIR1 and its substrates (Tan et al. 2007). 

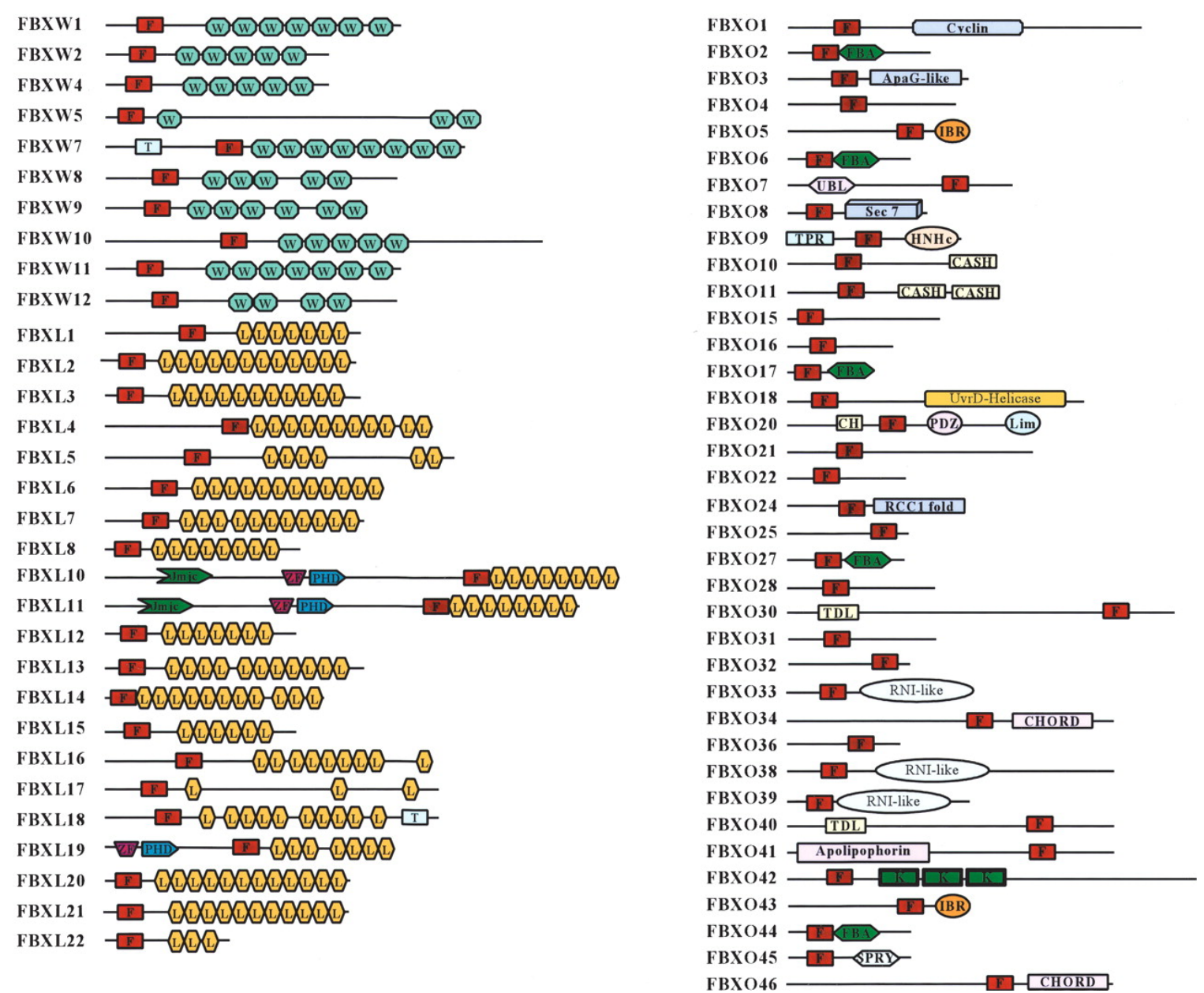

Figure 7: Domain structure of mammalian F-box proteins. Depending on the homology domains in addition to the F-box motif (F), F-box proteins are classified into three different subfamilies. Fbxw proteins contain WD40 repeats (WD), Fbxl proteins contain leucine-rich repeats $(\mathrm{L})$, and Fbxo proteins contain variable other domains. Picture is taken from (Jin et al. 2004). The following abbreviations are used: transmembrane domain (T), F-box-associated domain (FBA), between-ring domain (IBR), domain in carbohydrate binding proteins and sugar hydrolases (CASH), kelch repeat $(\mathrm{K})$, calponin homology domain $(\mathrm{CH})$, domain found in cupin metalloenzyme family (Jmjc), domain present in PSD-95, Dlg, and ZO-1 (PDZ), zinc-binding domain found in Lin11, Isl-1, and Mec-3 (Lim), HNH nuclease family (HNHc), novel eukaryotic zinc-binding domain (CHORD), tetratrico peptide repeat (TPR), structurally similar to bacterial ApaG (ApaG-like), apolipophorin-III-like fold (Apolipophorin), Ubiquitin-like fold (Ubl), Traf-domain like (TDL), structure similar to that of leucine-rich repeats in placental RNase inhibitor (RNI-like), regulator of chromatin condensation-1 fold (RCC1 fold).

\subsection{Physiological roles of F-box proteins}

As described above, regulation of target recognition by F-box proteins frequently involves target phosphorylation. This allows single F-box proteins to recognize 
many different target proteins in a spatially and temporally controlled manner. For example Fbxw1 ( $\beta$-TRCP), Fbxw7, and Fbxl1 (Skp2) have been shown to target tens of distinct substrates (summarized in (Skaar et al. 2009). Typically, a particular SCF E3 ligase complex can be linked to a general physiological function. For instance, $\mathrm{SCF}^{\mathrm{Fbxw1}}$ is considered to be a pro-survival factor, $\mathrm{SCF}^{\mathrm{Fbxw} 7}$ functions as a negative regulator of cell proliferation, and $\mathrm{SCF}^{\mathrm{Fbx} 11}$ is a positive regulator of the cell cycle by contributing to G1-S transition (Frescas and Pagano 2008; Welcker and Clurman 2008).

Although there are established roles for a small number of F-box proteins in many diverse pathways, the majority of the 69 mammalian F-box proteins have not been matched with any substrates. Moreover, some F-box proteins have not even been experimentally shown to be part of SCF complexes. In addition, there are some reports implicating F-box proteins from various species to have biological roles not directly related to SCF complexes (Hermand 2006), adding another layer of complexity to the functional analysis of F-box proteins.

\section{Fbxw5 - an unusual F-box protein}

The F-box and WD40 repeats containing protein 5 (Fbxw5) is a largely uncharacterized F-box protein of the Fbxw subfamily. As indicated by standard BLAST searches, Fbxw5 is conserved amongst vertebrates and, compared to the human protein, putative orthologues with around $40 \%$ identity can be identified in flies (e.g. Drosophila melongaster). According to standard prediction programs such as SMART and PFAM, the sequence of the $64 \mathrm{kDa}$ (566 residues) human Fbxw5 protein contains an N-terminal F-box motif and three recognizable WD40 repeats (Figure 8). While WD40 repeats are generally thought to form a $\beta$ propeller structure, it is currently unclear whether this is true for the ones of Fbxw5, since the formation of a $\beta$-propeller requires at least four to up to eight WD40 repeats (Paoli 2001).

Via its F-box domain, Fbxw5 can associate with Skp1 and Cul1 upon overexpression in HEK 293 cells (Winston et al. 1999; Dorrello et al. 2006). However, there is currently no experimental evidence for functional $\mathrm{SCF}^{\mathrm{Fbxw5}}$ 
complexes in vivo. Interestingly, recent studies have suggested that Fbwx5 can serve as a susbtrate recognition subunit (SRS) in context of a different CRL (Figure 8): upon overexpression of all three components, Fbwx5 co-purifies with DDB1 and Cul4A from HEK 293T cells (He et al. 2006). In this context, the Fbxw5-DDB1 interaction is likely mediated by two C-terminally located DDB1binding and WD40 (DWD) boxes (between residues 436-451 and 484-499) within Fbxw5. Further cell biological work by the same group suggests that Fbxw5 in context of a CRL4 complex (and independently from its F-box domain) mediates the degradation of the tuberous sclerosis complex protein 2 (TSC2) implicating a functional role of Fbxw5 in mTOR signaling (Hu et al. 2008). In another report, overexpressed Fbxw5 has been shown to interact with and negatively regulate the MAP3K TAK1 in an IL-1 $\beta$-dependent manner (Minoda et al. 2009). While the respective molecular mechanism remained elusive, the effect on TAK1 was again independent of the F-box domain of Fbxw5.

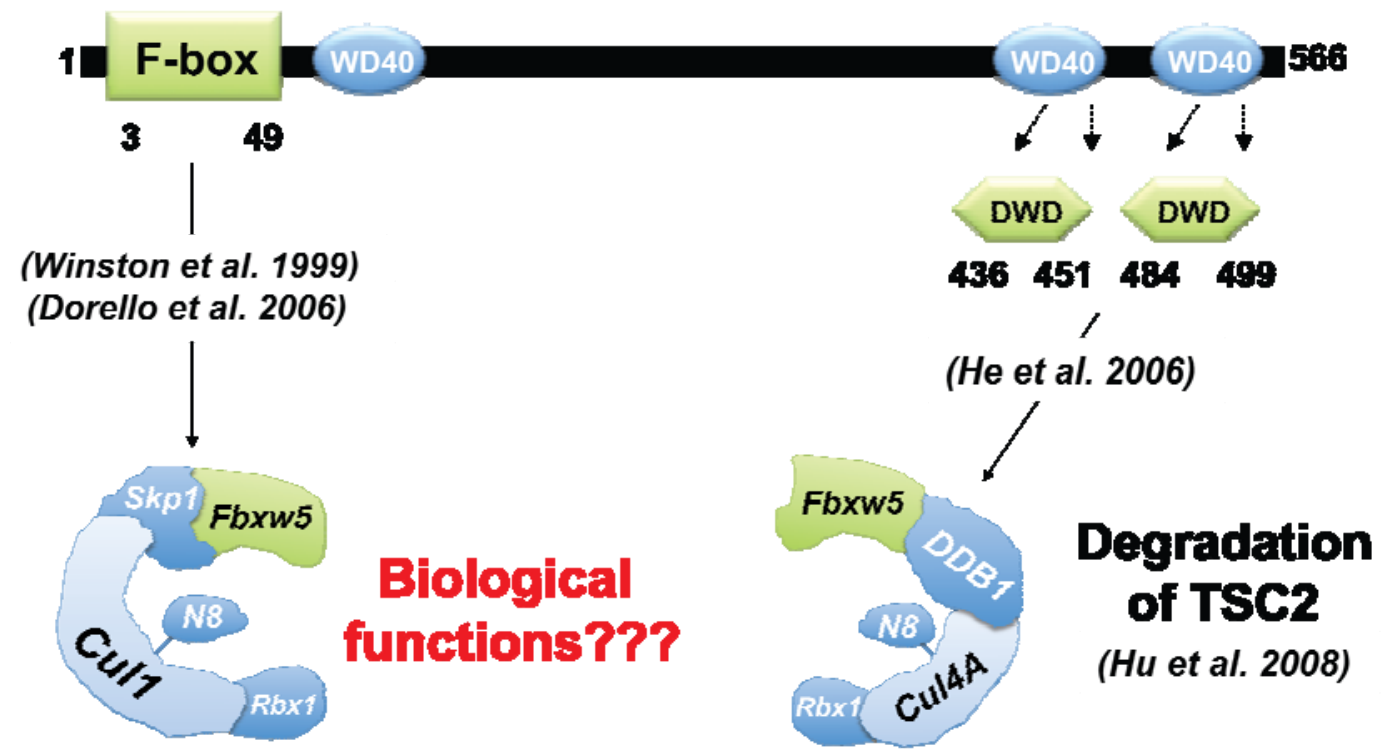

Figure 8: Domain structure of Fbxw5 and putative Fbxw5-containing CRL complexes. Sequence analysis with standard prediction programs such as PFAM and SMART reveal an Nterminal F-box domain and three WD40 repeats within Fbxw5. One of the WD40 repeats is located directly $\mathrm{C}$-terminal to the F-box motif, the other two repeats are located at the very Cterminus of the protein. The rest of the sequence contains no recognizable domains. Via its F-box domain, Fbwx 5 has been shown to interact with Skp1 and Cul1 upon overexpression. In addition, Fbxw5 can also interact with DDB1, most likely through the two WD40 repeats at the C-terminus, which each contain a DWD box. This way, Fbwx5 is thought to assemble into CRL4 complex that has been suggested to mediate the degradation of the tuberous sclerosis complex protein 2 (TSC2) in vivo. 
In conclusion, the biological functions of Fbxw5 have remained largely elusive. TSC2 is the only protein that has been suggested to be degraded by Fbxw5, which seems to mediate this process in a rather unexpected way: instead of utilizing an SCF complex, Fbxw5 degrades TSC2 in an F-box-independent manner by functioning as a SRS in a CRL4 complex. However, Fbxw5 can associate with Skp1 and Cul1 upon overexpression, indicating that the F-box domain is in principle functional and raising the possibility that Fbxw5 also exerts F-box dependent functions in context of SCF complexes within cells.

\section{Aims of this work}

Originally, Fbxw5 was classified into the F-box family of proteins due to the presence of an N-terminal F-box domain and its association with Skp1 in pull down assays (Winston et al. 1999). Since then, the functions and properties of Fbxw5 have remained largely elusive and have become even more enigmatic with the finding that it also is a DDB1 and Cul4-associated factor (DCAF, (He et al. 2006); raising the intriguing possibility that Fbxw5 acts as a substrate recognition subunit in context of two distinct Cullin-RING based E3 ligases (CRLs).

Hence, the aims of this work were to investigate the molecular composition of Fbxw5-containing complexes in vivo and to identify and characterize novel interaction partner and targets of this apparently versatile E3 ligase to obtain a deeper understanding of its biological functions. 


\section{MATERIALS AND METHODS}

\section{Materials}

\subsection{Technical equipment and software}

Technical equipment

Bacterial incubator Kelvitron $\mathrm{t}$

Bacterial incubator ISF-1-W

Cell culture incubator Hera cell

Cell culture incubator Incucell

Cell culture incubator multitron/multitherm

Centrifuge Allegra X-22R

Centrifuge RC3B Plus

Centrifuges 5415C, 5424, 5415, 5430, 5417R

Chromatography system Äkta Purifier

Electrophoresis Power Supply EPS300/301

Elektrophoresis and blotting chambers

EmulsiFlex-C5

Film developing machine Curix 60

HeraFreeze

Leica DM IL LED

NanoDrop ND1000

Rotors TLA-100.3, JS-5.2, Type45Ti,

Type60Ti, JA-20, Type70.1Ti

Rotor S45A

Scanner 4990 Photo

Sterile cell culture hood Herasafe

Thermocycler Primus
Heraeus

Kühner

Heraeus

MMM Medcenter

INFORS HT

Beckman Coulter

Sorvall

Eppendorf

GE Healthcare

Pharmacia Biotech

Workshop MPI, Martinsried and Biochemistry I, Göttingen

Avestin

Agfa

Thermo Scientific

Leica

Thermo Scientific

Beckman Coulter

Sorvall

Epson

Heraeus

MWG Biotech 
Thermocycler T3000 and Tprofessional

Biometra

Thermomixer Compact

Eppendorf

Ultracentrifuge Discovery ${ }^{\mathrm{TM}}$ SE, M120 SE

Sorvall

Ultracentrifuge Optima Max, Optima L-80 XP

Beckman Coulter

Vacuum pump LABOPORT N480.3FTP

KNF Neuberger

Vortex Genie 2

Scientific Industires

Software

Adobe Acrobat 9 pro

Adobe

Microsoft Office

Microsoft

Endnote X2

Thomson Reuters

Vector NTI

Invitrogen

\subsection{Consumables}

Autoradiography films

(Amersham Hyperfilm ${ }^{\mathrm{TM}} \mathrm{ECL}$ )

GE Healthcare

Cell culture consumables

Sarstedt, TPP

Centrifugal filter units

Millipore, Vivaspin

Dialysis tubing Spectra-Por

Roth

Disposable plastic columns Bio-Spin, Poly-Prep, Econo-Pac

Bio-Rad

Filter paper 3MM Whatman

Whatman

Gloves (Rotiprotect-LATEX, -NITRIL)

Roth

PROTRAN nitrocellulose

Schleicher \& Schuell

Reaction tubes

Sarstedt, Eppendorf

Sterile filters and membranes

$(0.22-0.45 \mu \mathrm{M})$

Millipore, Pall, Sartorius

\subsection{Chemicals, reagents and enzymes}

\#

Common chemicals were obtained from AppliChem, CARL ROTH GmbH, Merck, Serva, and Sigma-Aldrich. Some selected chemicals, reagents and enzymes are listed below: 
ANTI-FLAG M2 agarose, mouse

Sigma

Acrylamide solution (30 \%)

AppliChem

Aprotinin

Biomol

ATP

Sigma-Aldrich

BSA, fraction $\mathrm{V}$

AppliChem

Calf Intestine Phosphatase

NEB

CellfectinII® Reagent

Invitrogen

Cyanogen bromide-activated sepharose 4B

Sigma-Aldrich

DMEM (high glucose)

Gibco, PAA

DNA marker (1 kb)

Fermentas

dNTPs

Fermentas

ECL

Ethidium bromide

Express Five SFM

Invitrogen

FCS

Gibco

FuGENE6.0

Roche

G418

Sigma

Glutamine (cell culture grade)

Gibco

Glutathione sepharose FastFlow 4B

GE Healthcare

lodacetamide

Sigma

IPTG

Fermentas

JetPrime $^{\mathrm{TM}}$

Polyplus

Joklik's modified minimal essential medium

Sigma

Leupeptin

Biomol

Lipofectamine $^{2000}$ / Lipofectamine RNAiMAX

Invitrogen

3-Methyladenin (MA)

Sigma

MG132

Biomol

Monoclonal Anti-HA agarose, mouse clone HA-7

Sigma

$\mathrm{N}$-Ethylmaleimide (NEM)

Sigma 
Ni-NTA agarose

Qiagen

Protein A, Protein G agarose

Roche

Newborn calf serum, NCS

Gibco

Oligonucleotides

Sigma

OptiMEM

Invitrogen

Ovalbumin

Sigma

Pefa bloc

Roth, Sigma

Pepstatin

Biomol

Pfu Ultra, Pfu Turbo polymerase

Stratagene

Phosphatase inhibitor cocktail

Roche

Phusion polymerase

Finnzymes, NEB

PMSF

Sigma

Restriction enzymes

Fermentas, NEB

RNase inhibitor

Fermentas

SFM-900 II

Invitrogen

siRNA oligonucleotides

Ambion, Dharmacon

Sodium fluoride (NaF)

Sigma

Sodium orthovanadate

Sigma

T4 DNA ligase

Fermentas

Trypsin/EDTA

Gibco, PAA

1.4. Kits

NucleoBond ${ }^{\circledR}$ PC 100, PC500

Macherey \& Nagel

NucleoSpin ${ }^{\circledR}$ RNAll

Macherey \& Nagel

NucleoSpin $\AA$ Extract II

Macherey \& Nagel

QIAquick ${ }^{\circledR}$ Gel Extraction Kit

Qiagen

QIAquick ${ }^{\circledR}$ PCR Purification Kit

Qiagen

RevertAid $^{\mathrm{TM}}$ First Strand cDNA Synthesis Kit

Fermentas 
660nm Pierce Protein Detection Kit + lonic detergent compatibility reagent (IDCR)

\subsection{Buffers and stock solution}

Buffers and stock solutions were prepared in deioinzed water unless noted otherwise. Buffers were usually titrated with either sodium hydroxide or sodium hydrochloride. Stock solutions were prepared freshly or stored in aliquots at $-20^{\circ} \mathrm{C}$ unless indicated otherwise.

Commonly used buffers

DNA loading dye (6x)

$10 \mathrm{mM}$ Tris/HCl pH8, 50 mM EDTA, $1 \%$ (w/v) SDS, $30 \%$ (v/v) glycerol, $0.1 \%$ $(\mathrm{w} / \mathrm{v})$ bromophenol blue, $0.1 \%(\mathrm{w} / \mathrm{v})$ xylencyanol

Laemmli running buffer

NUPAGE LDS sample buffer

P1

P2

P3

Phosphate buffered saline (PBS)

PBS-Tween

RIPA buffer

TFB-I
$25 \mathrm{mM}$ Tris, $192 \mathrm{mM}$ glycine, 0,01\% (w/v) SDS; final, prepared as $10 \mathrm{x}$ stock solution for mass spectrometry analysis, commercially obtained from Invitrogen $50 \mathrm{mM}$ Tris $/ \mathrm{HCl} \mathrm{pH}$ 8.0, $10 \mathrm{mM}$ EDTA, $100 \mu \mathrm{g} / \mathrm{ml}$ RNase A $200 \mathrm{mM} \mathrm{NaOH}, 1 \%$ (v/v) SDS

$3 \mathrm{M} \mathrm{KOAc} \mathrm{pH} 5.5$ $140 \mathrm{mM} \mathrm{NaCl}, 2.7 \mathrm{mM} \mathrm{KCl}, 10 \mathrm{mM}$ $\mathrm{Na}_{2} \mathrm{HPO}_{4}, 1.5 \mathrm{mM} \mathrm{KH}_{2} \mathrm{PO}_{4}$, pH 7.5; final, prepared as $10 \mathrm{x}$ stock solution PBS supplemented with $0.2 \%(\mathrm{v} / \mathrm{v})$

Tween 20 $150 \mathrm{mM}$ sodium chloride, $1 \%$ (v/v) Triton$\mathrm{X} 100,0.5 \%(\mathrm{w} / \mathrm{v})$ sodium desoxycholate, $0.1 \%(\mathrm{w} / \mathrm{v})$ SDS, $50 \mathrm{mM}$ Tris-HCl, pH 8 $100 \mathrm{mM} \mathrm{RbCl}, 15 \%$ (v/v) glycerol, 0.5 
mM LiCl, pH 5.8

TFB-II

TAE buffer

Transport Buffer (TB)

Sumoylation assay buffer ( $A A B)$

SDS sample buffer

UT (extraction and purification) buffer

Western blot transfer buffer
$10 \mathrm{mM}$ MOPS pH 7, $10 \mathrm{mM} \mathrm{RbCl,} 75 \mathrm{mM}$ $\mathrm{CaCl}_{2}, 15 \%(\mathrm{v} / \mathrm{v})$ glycerol $40 \mathrm{mM}$ Tris acetate $\mathrm{pH}$ 7.7, 1 mM EDTA; final, prepared as $50 \mathrm{x}$ stock solution $110 \mathrm{mM}$ potassium acetate, $2 \mathrm{mM}$ magnesium acetate, $1 \mathrm{mM}$ EGTA, $20 \mathrm{mM}$ HEPES pH 7.3 titrated with potassium hydroxide; final, prepared as $10 x$ stock solution transport buffer supplemented with 0.2 $\mathrm{mg} / \mathrm{ml}$ ovalbumine, $0.05 \%(\mathrm{v} / \mathrm{v})$ Tween 20, 1 mM DTT, aprotinin, leupeptin, pepstatin $50 \mathrm{mM}$ Tris/HCl pH 6.8, $2 \%$ (w/v) SDS, $0.1 \%(\mathrm{w} / \mathrm{v})$ bromophenol blue, $10 \%(\mathrm{v} / \mathrm{v})$ glycerol, 100 mM DTT; final, prepared as $1 \mathrm{x}, 2 \mathrm{x}$ and $4 \mathrm{x}$ stock solutions

$8 \mathrm{M}$ urea, $50 \mathrm{mM}$ sodium phosphate $\mathrm{pH}$ 8 or $\mathrm{pH} 6.3$ $25 \mathrm{mM}$ Tris $/ \mathrm{HCl}, 193 \mathrm{mM}$ glycine, 20\% (v/v) methanol, 0,036\% (v/v) SDS; final, prepared as $10 x$ stock solution

Commonly used stock solutions

Ampicillin $100 \mathrm{mg} / \mathrm{ml}$

Ammonium chloride $1 \mathrm{M}$

Aprotinin (1000x) $1 \mathrm{mg} / \mathrm{mL}$

ATP

Chloramphenicol $100 \mathrm{mM}$ ATP, $100 \mathrm{mM}$ magnesium acetate, 20 mM HEPES $\mathrm{pH} 7.4$

Coomassie destainer $30 \mathrm{mg} / \mathrm{mL}$

Coomassie staining solution

$50 \%(\mathrm{v} / \mathrm{v})$ methanol, $10 \%(\mathrm{v} / \mathrm{v})$ acetic acid $50 \%(\mathrm{v} / \mathrm{v})$ methanol, $10 \%(\mathrm{v} / \mathrm{v})$ acetic acid, 2.5 $\%(w / v)$ Brill. Blue R250 
Dithiothreitol (DTT) $1 \mathrm{M}$

G418

$200 \mathrm{mg} / \mathrm{mL}$

lodoacetamide (100x)

$1 \mathrm{M}$, prepared freshly

Kanamycin

$50 \mathrm{mg} / \mathrm{mL}$

Leupeptin/Pepstatin (1000x)

$1 \mathrm{mg} / \mathrm{mL}$ each, in DMSO

MG132

$52.5 \mathrm{mM}$ in DMSO, stored at $-80^{\circ} \mathrm{C}$

N-ethylmaleimide (100x)

$1 \mathrm{M}$ in DMSO, prepared freshly

Pefa bloc, 100x

$100 \mathrm{mM}$

PMSF

$100 \mathrm{mM}$ in 2-propanol

Ponceau-S

0,5 \% (w/v) Ponceau-S, $1 \%$ (v/v) acetic acid

Puromycin

$1 \mathrm{mg} / \mathrm{mL}$ in PBS

\subsection{Media}

Bacterial media were sterilized by autoclaving; mammalian cell culture media were sterile-filtered.

Bacterial cell culture media

LB medium

$1 \%(\mathrm{w} / \mathrm{v})$ bacto tryptone, $0.5 \%(\mathrm{w} / \mathrm{v})$ yeast extract, $1 \%(\mathrm{w} / \mathrm{v}) \mathrm{NaCl}, \mathrm{pH} 7$, for agar plates LB medium was supplemented with $1.5 \%$ (w/v) bacto agar

SOC medium $2 \%(\mathrm{w} / \mathrm{v})$ tryptone, $5 \%(\mathrm{w} / \mathrm{v})$ yeast extract, $50 \mathrm{mM}$ $\mathrm{NaCl}, 2.5 \mathrm{mM} \mathrm{KCl}, 10 \mathrm{mM} \mathrm{MgCl}_{2}, 10 \mathrm{mM} \mathrm{MgSO}_{4}$

Insect cell culture media

Media (Express Five SFM and SFM-900 II, Invitrogen) and supplements for insect cell culture were commercially purchased.

\section{Mammalian cell culture media}

Jokliks medium for cultivation of suspension cells was prepared by dissolving one aliquot of Jokliks Minimum Essential Medium powder together with $20 \mathrm{~g}$ sodium hydrogen carbonate and $23.8 \mathrm{~g}$ HEPES (cell culture grade) in $10 \mathrm{~L}$ of autoclaved water. After $\mathrm{pH}$ titration to $\mathrm{pH} 7.1$ (sodium hydroxide), the medium was filtersterilized and stored at $4^{\circ} \mathrm{C}$. 
Media and supplements for adherent mammalian cell culture were commercially purchased from Invitrogen or PAA.

\subsection{Cell lines}

Bacterial strains

$\mathrm{DH} 5 \mathrm{a}$

F- $\phi 80$ lacZ $\Delta$ M15 (lacZYA-argF) U169 deoR recA1 endA1 hsdR7 $\left(r_{k^{-}}, m_{k}{ }^{+}\right)$phoA supE44 thi-1 gyrA96 relA1 $\lambda$ -

$\mathrm{BI} 21$ (DE3)

F- ompT hsdS ${ }_{B}\left(r_{B^{-}}-m_{B^{-}}\right)$gal dcm $\lambda(D E 3)$

BI21 (DE3) pLysS

F- ompT hsdS ${ }_{B}\left(r_{B^{-}}-m_{B^{-}}\right)$gal dcm $\lambda(D E 3)$, $\operatorname{pLysS}\left(\mathrm{Cm}_{\mathrm{R}}\right)$

BI21 (DE3) pLysE

F- ompT hsdS $S_{B}\left(r_{B^{-}} m_{B^{-}}\right)$gal dem $\lambda(D E 3)$, pLysE $\left(\mathrm{Cm}_{\mathrm{R}}\right)$

Rosetta (DE3)

F- ompT hsdSB $\left(\mathrm{r}_{\mathrm{B}^{-}} \mathrm{m}_{\mathrm{B}^{-}}\right)$gal dcm (DE3) pRARE $\left(\mathrm{Cm}_{\mathrm{R}}\right)$

Rosetta2 (DE3)

F- ompT hsdSB $\left(r_{B^{-}}-m_{B^{-}}\right)$gal dcm (DE3) pRARE2 $\left(\mathrm{Cm}_{\mathrm{R}}\right)$

Arctic Express $^{T M}$ (DE3) RIL $\quad$ E. coli B F- ompT hsdSB( $\left.r_{B^{-}}-m_{B^{-}}\right)$dcm Tet $^{r}$ gal $\lambda$ (DE3) endA Hte [cpn10 cpn60 Gentr] [argU ileY leuW Str']

DH10Bac

F- mcrA $\Delta$ (mrr-hsdRMS-mcrBC) $\varphi 80$ lacZ $\Delta$ M15 $\Delta$ lacX74 recA1 endA1 araD139 $\Delta$ (ara,leu)7697 galU galK $\lambda^{-}$rpsL nupG / pMON14272 / pMON7124

Insect cell lines

SF-9 Cell line derived from pupal ovarian tissue of Spodoptera frugiperda High Five Cell line derived from Trichoplusia ni egg cell homogenates

Mammalian cell lines

HeLa (obtained from Mary Osborn) Human cervix carcinoma cell line HeLa suspension cells (CSH HeLa strain) Human cervix carcinoma cell line HEK 293T Human embryonic kidney cell line 
HeLa S3 pellets (RELIATech)

Human cervix carcinoma cell line

\subsection{Oligonucleotides}

\begin{tabular}{|c|c|c|}
\hline \multicolumn{3}{|c|}{ DNA oligonucleotides for cloning } \\
\hline \# & name & Sequence $\left(5^{\prime} \rightarrow 3^{\prime}\right)$ \\
\hline 1249 & Fbxw5_BamHI_for & GTCGC GGATCC ATG GACGAGGGCGGCACGCCCCTG \\
\hline 1250 & Fbxw5_Notl_rev & CGAGT GCGGCCGC TCA GCGCCTCTGGCTGGCAAGC \\
\hline 1798 & Cdc34_BamHI_for & CCGG GGATCC ATGGCTCGGCCGCTAGTGCCCAGCTCGC \\
\hline 1799 & Cdc34_Xhol_rev & GCGC CTCGAG TCA GGACTCCTCCGTGCCAGAGTCATCC \\
\hline 1805 & Fbxw5_HindIII_for & CCGG AAGCTT ATG GACGAGGGCGGCACGCCCCTGCTCC \\
\hline 1806 & Fbxw5_Xhol_rev & CGCG CTCGAG TCAGCGCCTCTGGCTGGCAAGCCAGG \\
\hline 1809 & Ubch5c_BamHI_for & CCGGG GATCC ATG GCGCTGAAACGGATTAATAAGGAACTTAG \\
\hline 1810 & Ubch5c_Xhol_rev & GCGC CTCGAG TTA CATGGCATACTTCTGAGTCCATTCCCG \\
\hline 1820 & Fbxw5 N80_BamHI_for & CCGG GGATCC ATG CCCTGCGTGGAGGTGCAGACG \\
\hline 1866 & Eps8_BamHI_for & CCGG GGATCC ATG AATGGTCATATTTCTAATCATCCCAG \\
\hline 1867 & Eps8_Xhol_rev & CCTT CTCGAG TTA GTGACTGCTTCCTTCATCAAAAG \\
\hline 1893 & mFbxw5_BamHI_AN81_for & CCGG GGATCC TGTGTGGAGGTGCAGACAC \\
\hline 1894 & mFbxw5_Notl_stop_rev & GTAA GCGGCCGC TCA GCGCCTATGGCTGGC \\
\hline 1895 & mFbxw5_BamHI_for & CGTT GGATCC GATGGATGAGGGGGG \\
\hline 1896 & mFbxw5_Notl_468_stop_rev & CGTT GCGGCCGC TCAGGCACGGTGCGCTCGAAGAG \\
\hline 1907 & Nek9_STOP_Notl_rev & CCGG GCGGCCGC CTAGAGGCTGGGTCTAC \\
\hline 1919 & Nek9_Bglll_for & CCGG AGATCT ATGTCGGTGCTGGGCGAG \\
\hline 1920 & mFbxw5_for_Notl & CCGG GCGGCCGC TC ATGGATGAGGGGGGCCTG \\
\hline 1921 & mFbxw5_rev_BamHI & CAGC GGATCC TCA GCGCCTATGGCTGGC \\
\hline 1945 & mFbxw5 $\Delta$ N81_for_Notl & CCGG GCGGCCGC TC ATG TGTGTGGAGGTGCAGACAC \\
\hline \multicolumn{3}{|c|}{ DNA oligonucleotides for site-directed mutagenesis } \\
\hline 1814 & Fbxw5_R498A_for & CGGCACGGCTACATCTGGGACGCCCACTACAACATCTGTCTGGC \\
\hline 1815 & Fbxw5_R498A_rev & GCCAGACAGATGTTGTAGTGGGCGTCCCAGATGTAGCCGTGCCG \\
\hline 1882 & Eps8_K554R_for & GTGAGCTCTCGGTTCTAAGGGATGATATTTTAGAGATAC \\
\hline 1883 & Eps8_K554R_rev & GTATCTCTAAAATATCATCCCTTAGAACCGAGAGCTCAC \\
\hline \multicolumn{3}{|c|}{ DNA oligonucleotides for Real Time PCR } \\
\hline 1898 & Fbxw5_RT_for & GCAGTTCTACCGCTACTACCAG \\
\hline 1899 & Fbxw5_RT_rev & AGATGGTCAGGTCGTTGCTC \\
\hline 1900 & 36B4_RT_for & GATTGGCTACCCAACTGTTG \\
\hline 1901 & 36B4_RT_rev & CAGGGGCAGCAGCCACAAA \\
\hline 1902 & GAPDH_RT1_for & GAAGGTCGGAGTCAACGGATTTG \\
\hline 1903 & GAPDH_RT1_rev & CAGAGATGATGACCCTTTTGGCTC \\
\hline 1939 & Eps8_RT_for & CAGAATCCTAGTGCTGCAGA \\
\hline 1940 & Eps8_RT rev & CTGCCGTTCATCACCATTGA \\
\hline
\end{tabular}




\begin{tabular}{|c|c|c|}
\hline 1941 & GAPDH_RT2_for & AAGGTGAAGGTCGGAGT \\
\hline 1942 & GAPDH_RT2_rev & GAAGATGGTGATGGGATTTC \\
\hline \multicolumn{3}{|c|}{ DNA oligonucleotides for sequencing } \\
\hline 1811 & seq_Fbxw5_Cterm & CAGTCCCCAGGAGCAGGAGC \\
\hline 1257 & Fbxw5_seq1 & CCAGCGGCCATGTCCTGGTA \\
\hline 1258 & Fbxw5_seq2 & CCGAGACCAGCCTCATCTCG \\
\hline 1259 & Fbxw5_seq3 & GCAGCCGCTTCGACAGCCCT \\
\hline 1260 & Fbxw5_seq4 & CAGATCGGCATCAAGCAGAT \\
\hline 1261 & Fbxw5_seq5r & ACCGCCACTACAACATCTGT \\
\hline 1862 & M13_for(40) & GTTTTCCCAGTCACGAC \\
\hline 1863 & M13_rev & CAGGAAACAGCTATGAC \\
\hline 1877 & h_Eps8_854r & ССTTATTCCATCATCAACAG \\
\hline 1878 & h_Eps8_1117 & GTTAAGGCAAACCTAATTAG \\
\hline 1879 & h_Eps8_1546 & GGTGTTTTAACGCTGCGG \\
\hline 1880 & h_Eps8_1982 & CTGTGGCAAATGTAGCAG \\
\hline 1881 & h_Eps8_2436 & GATGGAGTATGGCCCAAGAC \\
\hline 1912 & Nek9_771 & CCATGGCTGAGACGCTTGTGG \\
\hline 1913 & Nek9_1541 & GTTGCTGGCCCTGAAGTGCTAG \\
\hline 1914 & Nek9_2201 & GCAATGACCCCCACAGAGAG \\
\hline 1915 & Nek9_2680 & CCTGCCCTATGAAGAGC \\
\hline 1916 & mFbxw5_771 & СTCCTGCTCAGTGCTGTGGCTC \\
\hline 1917 & mFbxw5_1211 & GCCTCACGTACTCACCGCATC \\
\hline 1918 & mFbxw5_1651 & CTAGCCAAGCTGCGGCATGAG \\
\hline \multicolumn{3}{|c|}{ DNA oligonucleotides for ligation into pSUPER vector } \\
\hline 1933 & Fbxw5_sh_1_forward & $\begin{array}{l}\text { gatcccC CCACAGGCGCCAAGAGCAA ttcaagaga } \\
\text { TTGCTCTTGGCGCCTGTGG ttttggaaa }\end{array}$ \\
\hline 1934 & Fbxw5_sh_1_reverse & $\begin{array}{l}\text { agctttccaaaa CCACAGGCGCCAAGAGCAA tctcttgaa } \\
\text { TTGCTCTTGGCGCCTGTGG ggg }\end{array}$ \\
\hline 1935 & Fbxw5_sh_3_forward & $\begin{array}{l}\text { gatcccc CCCTACAACTGGAGCTACA ttcaagaga } \\
\text { TGTAGCTCCAGTTGTAGGG tttttggaaa }\end{array}$ \\
\hline 1936 & Fbxw5_sh_3_reverse & $\begin{array}{l}\text { agctttccaaaa CCCTACAACTGGAGCTACA tctcttgaa } \\
\text { TGTAGCTCCAGTTGTAGGG ggg }\end{array}$ \\
\hline 1937 & Fbxw5_sh_4_forward & $\begin{array}{l}\text { gatcCCC GGACCACGTCATAGACATA ttcaagaga } \\
\text { TATGTCTATGACGTGGTCC tttttggaaa }\end{array}$ \\
\hline 1938 & Fbxw5_sh_4_reverse & $\begin{array}{l}\text { agcttttccaaaaa GGACCACGTCATAGACATA tctcttgaa } \\
\text { TATGTCTATGACGTGGTCC ggg }\end{array}$ \\
\hline 1943 & nt_1_forward & $\begin{array}{l}\text { gatcccC TAGCGACTAAACACATCAA ttcaagaga } \\
\text { TTGATGTGTTTAGTCGCTA ttttggaaa }\end{array}$ \\
\hline 1944 & nt_1_reverse & $\begin{array}{l}\text { agcttttccaaaaa TAGCGACTAAACACATCAA tctcttgaa } \\
\text { TTGATGTGTTTAGTCGCTA ggg }\end{array}$ \\
\hline
\end{tabular}

\subsection{Vectors and plasmids}

\begin{tabular}{|l|l|l|l|}
\hline \multicolumn{4}{|l|}{ Vectors for bacterial expression } \\
\hline name & features & antibiotic resistance & origin \\
\hline pET11a & & Ampicillin & Novagen \\
\hline
\end{tabular}




\begin{tabular}{|c|c|c|c|}
\hline pET28a & N-term. His-tag & Kanamycin & Novagen \\
\hline pGEX-6P3 & $\begin{array}{l}\text { N-term. GST- } \\
\text { tag, Prescission } \\
\text { cleavage site }\end{array}$ & Ampicillin & GE Healthcare \\
\hline \multicolumn{4}{|c|}{ Vectors for insect cell expression } \\
\hline pFASTBac_HT_B & $\begin{array}{l}\text { N-term. His-tag, } \\
\text { TEV cleavage } \\
\text { site }\end{array}$ & Ampicillin & Invitrogen \\
\hline \multicolumn{4}{|c|}{ Vectors for mammalian cell expression } \\
\hline pCDNA3.1(+) & & Ampicillin, G418 & Invitrogen \\
\hline pIRES-hrGFP1a & $\begin{array}{l}\text { C-term. flag-tag, } \\
\text { internal } \\
\text { ribosomal entry } \\
\text { site for gene of } \\
\text { interest }\end{array}$ & Ampicillin & Stratagene \\
\hline pSUPER-puro-epi & $\begin{array}{l}\text { Episomal } \\
\text { replication of } \\
\text { plasmid in } \\
\text { cytoplasm }\end{array}$ & Ampicillin, Puromycin & $\begin{array}{l}\text { (Azzalin and } \\
\text { Lingner 2006) }\end{array}$ \\
\hline
\end{tabular}

\begin{tabular}{|c|c|c|}
\hline \multicolumn{3}{|c|}{ Plasmids for bacterial expression } \\
\hline name & species & source \\
\hline pET28a-His-TEV & tobacco etch virus & Ralph Kehlenbach* \\
\hline pGEX-4T1-GST-Prescission & human rhinovirus & Achim Dickmanns ${ }^{\#}$ \\
\hline pGEX6P3-GST-Fbxw5 & human & This work \\
\hline pET28a-His-Fbxw5 & human & This work \\
\hline pET28a-His-Ubiquitin & human & This work \\
\hline pET28a-His-UbiquitinK48R & human & This work \\
\hline pET28a-His-UbiquitinK29,48,63R & human & This work \\
\hline pGEX4X1-UbcH5B & human & Andrea Pichler ${ }^{\wedge}$ \\
\hline pGEX4X1-Cdc34 & human & Andrea Pichler ${ }^{\wedge}$ \\
\hline pGEX6P3-UbcH5B & human & This work \\
\hline pGEX6P3-Cdc34 & human & This work \\
\hline pET22b-Ube1-His & human & Andrea Pichler ${ }^{\wedge}$ \\
\hline pAL-His-Cul1-NTD & human & (Li et al. 2005) \\
\hline pGEX-4T1-Rbx1-rbs-Cul1-CTD & human & (Li et al. 2005) \\
\hline pGEX-4T1-Skp2 $\Delta N$-rbs-Skp1 $1 \Delta \Delta^{\partial}$ & human & (Li et al. 2005) \\
\hline pGEX-4T1-Fbxw5-rbs-Skp1 $\Delta \Delta$ & human & This work \\
\hline pGEX-4T1-modTEV-Skp1 & human & $\begin{array}{l}\text { Unpublished plasmid from } \\
\text { Matthew Calabrese }\end{array}$ \\
\hline pRSFDuet-HisMBP-m-Fbxw5 & mouse & $\begin{array}{l}\text { Unpublished plasmid from } \\
\text { Matthew Calabrese }\end{array}$ \\
\hline
\end{tabular}




\begin{tabular}{|c|c|c|}
\hline \multicolumn{3}{|l|}{ Plasmids for insect cell expression } \\
\hline pFASTBacHT-B-His-Fbxw5 & human & This work \\
\hline \multicolumn{3}{|l|}{ Plasmids for mammalian cell expression } \\
\hline pCDNA3.1-flag-Usp25 & human & (Meulmeester et al. 2008) \\
\hline pCDNA3.1-HA-Usp25 & human & (Meulmeester et al. 2008) \\
\hline pCDNA3.1-HA-Ubiquitin & human & (Haglund et al. 2003) \\
\hline pCDNA3.1-HA-UbiquitinK48R & human & (Haglund et al. 2003) \\
\hline pCDNA3.1-HA-UbiquitinK29,48,63R & human & (Haglund et al. 2003) \\
\hline pCDNA3.1-flag-Fbxw5 & human & This work \\
\hline pCDNA3.1-flag-Fbxw5R498A & human & This work \\
\hline pCDNA3.1-flag-Fbxw5 $\Delta \mathrm{F}$ box $\quad \Delta \mathrm{N} 80$ ) & human & This work \\
\hline pCDNA3.1-HA-Fbxw5 & human & This work \\
\hline pCDNA3.1-flag-m-Fbxw5 & mouse & This work \\
\hline $\begin{array}{l}\text { pCDNA3.1-flag-m- } \\
\text { Fbxw5 } \mathrm{F} \text { box } \quad \Delta \mathrm{N} 81)\end{array}$ & mouse & This work \\
\hline pCDNA3.1-flag-m-Fbxw5-1-468 & mouse & This work \\
\hline pCDNA3.1-HA-m-Fbxw5 & mouse & This work \\
\hline pIRES-hrGFP1a-m-Fbxw5 & mouse & This work \\
\hline $\begin{array}{l}\text { pIRES-hrGFP1a-m- } \\
\text { Fbxw5 } \Delta \mathrm{F} \text { box } \quad \Delta \mathrm{N} 81)\end{array}$ & mouse & This work \\
\hline pDNR-LIB-h-Nek9 & human & Imagenes \\
\hline pCDNA3.1-flag-Nek9 & human & This work \\
\hline pCDNA3.1-HA-Nek9 & human & This work \\
\hline pCDNA3.1-flag-h-Eps8 & human & This work \\
\hline pCMV5-Nek9-wt & human & (Belham et al. 2003) \\
\hline pCMV5-Nek9-K81M & human & (Belham et al. 2003) \\
\hline pCMV5-Nek9- $\Delta 347-732$ & human & (Belham et al. 2003) \\
\hline pCDNA3.1-HA-h-Eps8 & human & This work \\
\hline pCDNA3.1-flag-h-Eps8K554R & human & This work \\
\hline pCDNAHisMax4-m-Eps8 & mouse & (Disanza et al. 2006) \\
\hline pSUPER-puro-epi-shFbxw5-1 & targets human & This work \\
\hline pSUPER-puro-epi-shFbxw5-3 & targets human & This work \\
\hline pSUPER-puro-epi-shFbxw5-4 & targets human & This work \\
\hline pSUPER-puro-epi-shnt-1 & non-targeting & This work \\
\hline
\end{tabular}

${ }^{\partial} \operatorname{Skp} 1 \Delta \Delta=$ human Skp1 version with two internal deletions (residues 38 to 43 and 71 to 82 )

${ }^{*}$ Dr. habil. Ralph Kehlenbach, University of Göttingen

\# Dr. Achim Dickmanns, University of Göttingen

AAndrea Pichler, Max Planck Institute for Immunology, Freiburg

${ }^{`}$ Matthew Calabrese, St. Judes Children's Research Hospital, Memphis,TN, USA 


\subsection{Peptides}

\begin{tabular}{|l|l|l|}
\hline Peptide & Sequence & Source \\
\hline Flag-peptide & DYKDDDDK & Pepscan \\
\hline HA-peptide & YPYDVPDYA & Pepscan \\
\hline
\end{tabular}

\subsection{Recombinant proteins}

\begin{tabular}{|l|l|}
\hline Protein & Source \\
\hline His-TEV & Purified within this work \\
\hline GST-Prescission & Purified within this work \\
\hline GST-/His-Fbxw5 (denatured) & Purified within this work \\
\hline His-Ubiquitin & Purified within this work \\
\hline His-UbiquitinK29,48,63R & Purified within this work \\
\hline Cdc34 & Purified within this work \\
\hline UbcH5B & Purified within this work \\
\hline hFbxw5/Skp1 $\Delta \Delta$ & Purified within this work \\
\hline mFbxw5/Skp1 $\Delta \Delta$ & Purified within this work \\
\hline Nedd8 Cul1/Rbx1 & Brenda Schulman* (Duda et al. 2008) \\
\hline His-Eps8 & Giorgio Scita* (Disanza et al. 2006) \\
\hline HA-Nek9 & Purified within this work $^{*}$ \\
\hline Flag-Fbxw5 & Purified within this work \\
\hline SUMO1 $\Delta$ C4 & General stock \\
\hline SUMO2 $\Delta C 11$ & General stock \\
\hline RanGAP1 & General stock \\
\hline Ovalbumin & Sigma \\
\hline
\end{tabular}

* Collaborators, who provided recombinant protein

\# These proteins were purified by members of the Melchior lab and are available as common protein stocks in the lab

${ }^{\diamond}$ Former member of the Melchior lab

\subsection{Antibodies}

Primary antibodies

\begin{tabular}{|l|l|l|l|l|}
\hline Antibody & Immunogen & $\begin{array}{l}\text { Origin / } \\
\text { reference }\end{array}$ & Concentration & $\begin{array}{l}\text { Dilution } \\
\text { for WB }\end{array}$ \\
\hline rabbit $\alpha$-Fbxw5 & $\begin{array}{l}\text { denatured GST-/ His- } \\
\text { Fbxw5 }\end{array}$ & $\begin{array}{l}\text { Produced in } \\
\text { this work }\end{array}$ & $0.6 \mathrm{mg} / \mathrm{mL}$ & $1: 5000$ \\
\hline mouse $\alpha$-Eps8 & $\begin{array}{l}\text { C-terminus of mEps8 } \\
\text { (aa 628-821) }\end{array}$ & BD Bioscience & $1.0 \mathrm{mg} / \mathrm{mL}$ & $\begin{array}{l}1: 1000- \\
1: 5000\end{array}$ \\
\hline
\end{tabular}




\begin{tabular}{|c|c|c|c|c|}
\hline goat $\alpha$-Nek9 & $\begin{array}{l}\text { N-terminal peptide of } \\
\text { hNek9 }\end{array}$ & Santa Cruz & $0.2 \mathrm{mg} / \mathrm{mL}$ & $1: 500$ \\
\hline $\begin{array}{l}\text { mouse } \alpha \text {-Cul1 } \\
\text { clone } 2 \mathrm{H} 4 \mathrm{C} 9\end{array}$ & $\begin{array}{l}\text { C-terminal peptide of } \\
\text { hCul1 }\end{array}$ & Zymed & $0.5 \mathrm{mg} / \mathrm{mL}$ & $1: 2000$ \\
\hline rabbit $\alpha-C u l 4 A$ & $\begin{array}{l}\text { N-terminal peptide of } \\
\text { hCul } 4 \mathrm{~A}\end{array}$ & Cell Signaling & unknown & $1: 2000$ \\
\hline goat $\alpha$-Skp1 & $\begin{array}{l}\text { C-terminal peptide of } \\
\text { hSkp1 }\end{array}$ & Santa Cruz & $0.2 \mathrm{mg} / \mathrm{mL}$ & $\begin{array}{l}1: 250- \\
1: 500\end{array}$ \\
\hline rabbit $\alpha-R b x 1$ & PLDNREWEFQKYGH & $\begin{array}{l}\text { Invitrogen / } \\
\text { Biosource }\end{array}$ & $1.0 \mathrm{mg} / \mathrm{mL}$ & $1: 1000$ \\
\hline rabbit $\alpha-D D B 1$ & $\begin{array}{l}\text { C-terminus of hDDB1 } \\
\text { (aa 1100-1140) }\end{array}$ & $\begin{array}{l}\text { Bethyl } \\
\text { Laboratories }\end{array}$ & $0.2 \mathrm{mg} / \mathrm{mL}$ & $\begin{array}{l}1: 1000- \\
1: 10,000\end{array}$ \\
\hline $\begin{array}{l}\text { rabbit } \alpha-\text { TSC2 } \\
(\mathrm{C} 20)\end{array}$ & C-terminus of hTSC2 & Santa Cruz & $0.2 \mathrm{mg} / \mathrm{mL}$ & $\begin{array}{l}1: 1000- \\
1: 5000\end{array}$ \\
\hline rabbit $\alpha$-TRIM28 & $\begin{array}{l}\text { C-terminal peptide of } \\
\text { hTRIM28 }\end{array}$ & Cell Signaling & unknown & $1: 1000$ \\
\hline $\begin{array}{l}\alpha-A C A P 2 \\
(\mathrm{C} 20)\end{array}$ & C-terminus of hACAP2 & Santa Cruz & $0.2 \mathrm{mg} / \mathrm{mL}$ & $1: 500$ \\
\hline $\begin{array}{l}\text { mouse } \alpha-\mathrm{HA} \\
\text { clone HA. } 11\end{array}$ & CYPYDVPDYASL & Covance & $1.0 \mathrm{mg} / \mathrm{mL}$ & $1: 1000$ \\
\hline $\begin{array}{l}\text { mouse } \alpha-\text { FLAG }^{\circledR} \\
\text { clone M2 }\end{array}$ & $\begin{array}{l}\text { Peptide containing } \\
\text { DYKDDDK sequence }\end{array}$ & Sigma & $1.0 \mathrm{mg} / \mathrm{mL}$ & $\begin{array}{l}1: 500- \\
1: 1000\end{array}$ \\
\hline $\begin{array}{l}\text { mouse } \alpha \text { - } \alpha \text { tubulin } \\
\text { clone DM1A }\end{array}$ & $\begin{array}{l}\text { Chicken brain } \\
\text { microtubules, specific } \\
\text { for } \alpha \text { tubulin }\end{array}$ & Sigma & ascites & $\begin{array}{l}1: 10,000- \\
1: 100,000\end{array}$ \\
\hline goat $\alpha$-RanGAP1 & mRanGAP1 & $\begin{array}{l}\text { Melchior lab } \\
\text { (Pichler et al. } \\
\text { 2002) }\end{array}$ & $0.8 \mathrm{mg} / \mathrm{mL}$ & $1: 3000$ \\
\hline $\begin{array}{l}\text { mouse } \alpha \text {-Ubiquitin } \\
\text { clone 4PD1 }\end{array}$ & Ubiquitin from cow & Santa Cruz & $0.2 \mathrm{mg} / \mathrm{mL}$ & $1: 500$ \\
\hline
\end{tabular}

\section{Secondary antibodies}

Horseradish peroxidase-conjugated antibodies raised in donkey against IgGs from goat, mouse, or rabbit were obtained from Dianova. They were used for western blot analysis in dilutions ranging from 1:5,000 to 1:10,000.

\subsection{SiRNAs}

Small interfering RNAs were ordered as 2'-deprotected and pre-annealed duplexes in lyophilized form. They were resuspended in RNAse-free water according to the manufacturer's guidelines and stored in small aliquots at concentrations ranging from $20-100 \mu \mathrm{M}$ at $-20^{\circ} \mathrm{C}$.

siFbxw5 \#1-3 were designed within this work by using the online tool siRNA target finder from Ambion (http://www.ambion.com/techlib/misc/siRNA finder.html). 


\begin{tabular}{|c|c|c|c|c|}
\hline name & Target gene & Sense $\left(5^{\prime} \rightarrow 3^{\prime}\right)$ & Antisense $\left(5^{\prime} \rightarrow 3^{\prime}\right)$ & $\begin{array}{l}\text { Sequence } \\
\text { Origin }\end{array}$ \\
\hline Fbxw5 1 & human Fbxw5 & $\begin{array}{l}\text { CCACAGGCGCCAA } \\
\text { GAGCAAdTdT }\end{array}$ & $\begin{array}{l}\text { UUGCUCUUGGCG } \\
\text { CCUGUGGdTdT }\end{array}$ & $\begin{array}{l}\text { Designed } \\
\text { within this } \\
\text { work }\end{array}$ \\
\hline Fbxw5 2 & human Fbxw5 & $\begin{array}{l}\text { CGGGAGAGGUGGA } \\
\text { GAUGCUdTdT }\end{array}$ & $\begin{array}{l}\text { AGCAUCUCCACCU } \\
\text { CUCCCGdTdT }\end{array}$ & $\begin{array}{l}\text { Designed } \\
\text { within this } \\
\text { work }\end{array}$ \\
\hline Fbxw5 3 & human Fbxw5 & $\begin{array}{l}\text { CCCUACAACUGGA } \\
\text { GCUACAdTdT }\end{array}$ & $\begin{array}{l}\text { UGUAGCL } \\
\text { UGUAGGC }\end{array}$ & $\begin{array}{l}\text { Designed } \\
\text { within this } \\
\text { work }\end{array}$ \\
\hline Fbxw5 4 & Fbxw5 & $\begin{array}{l}\text { GGACCACGUCAUA } \\
\text { GACAUAtt }\end{array}$ & $\begin{array}{l}\text { UAUGUCUAUCAG } \\
\text { UGGUCCag }\end{array}$ & $\begin{array}{l}\text { Pre- } \\
\text { designed } \\
\text { (Applied } \\
\text { Biosystmes) }\end{array}$ \\
\hline Cul4a 1 & human Cul4A & $\begin{array}{l}\text { UCCGAGAC } \\
\text { UdTdT }\end{array}$ & $\begin{array}{l}\text { UCG } \\
d T\end{array}$ & $\begin{array}{l}\text { (Hu et al. } \\
2008)\end{array}$ \\
\hline Cul4b 1 & human Cul4B & $\begin{array}{l}\text { AAGCCUAAAUUACC } \\
\text { AGAAAdTdT }\end{array}$ & $\begin{array}{l}\text { UUUCUGGUAAUU } \\
\text { UAGGCUUdTdT }\end{array}$ & $\begin{array}{l}\text { (Hu et al. } \\
2008)\end{array}$ \\
\hline
\end{tabular}

\section{Methods}

Standard procedures in molecular biology, biochemistry, and cell biology were performed according to basic methods described in Molecular Cloning: $A$ Laboratory Manual, Maniatis, T., Fritsch, E.F. \& Sambrook, J. (Cold Spring Harbor Laboratory, New York, 1982), Current Protocols in Protein Science, Coligan, J.E., Dunn B.M., Speicher, D.W., Wingfield, P.T. (John Whiley \& Sons, 2003), and Current Protocols in Cell Biology, Bonifacino, J.S., Dasso, M., Harford, J.B., Lippincott-Schwartz, J., Yamada, K.M. (John Whiley \& Sons, 2000).

\subsection{Molecular biology techniques}

\subsubsection{Preparation of chemically competent bacteria}

Chemically competent E.coli were prepared using a $\mathrm{RbCl}$-based protocol from a $200 \mathrm{ml}$ growing culture with an optical density $\mathrm{OD}_{600}$ of 0.5 . Bacteria were incubated on ice for $10 \mathrm{~min}$ and harvested by centrifugation with $5,000 \mathrm{xg}$ at $4^{\circ} \mathrm{C}$. The cell pellet was resuspended in $200 \mathrm{ml}$ ice-cold TFB-I buffer followed by incubation on ice for $2 \mathrm{~h}$. Cells were again collected with $5,000 \mathrm{~g}$ at $4^{\circ} \mathrm{C}$, resuspended in $8 \mathrm{ml}$ of sterile cold TFB-II buffer, flash frozen in $100 \mu \mathrm{L}$ aliquots, and stored at $-80^{\circ} \mathrm{C}$. 


\subsubsection{Transformation of competent bacteria}

Chemically competent E.coli were thawed on ice and incubated with Plasmid DNA for 20 min on ice. Incorporation of the DNA was achieved by heat-shock at $42^{\circ} \mathrm{C}$ for 60 seconds, followed by incubation on ice for $5 \mathrm{~min}$. For regeneration, bacteria were supplemented with 700 /L LB medium and incubated in a shaker at $37^{\circ} \mathrm{C}$ for $1 \mathrm{~h}$. Finally, transformed cells were plated on LB agar plates supplemented with the respective antibiotic for the transformed plasmid.

\subsubsection{Plasmid DNA preparation}

Typically, small amounts of plasmid DNA (e.g. for clone screening, mutagenesis, and DNA sequencing) were prepared from DH5 $\alpha$ by alkaline lysis (Birnboim and Doly 1979) and subsequent precipitation. For this, $2-4 \mathrm{ml}$ overnight cultured DH5 $\alpha$ were harvested by centrifugation with $3,000 \times g$ at room temperature for 5 min and resuspended in 300 /L buffer P1. Cells were lyzed by SDS under alkaline conditions by the addition of 300 /L P2 and incubated for 5 minutes at room temperature before $\mathrm{pH}$ neutralization and precipitation of proteins and chromosomal DNA by the addition of 300 (L buffer P3. The lysate was cleared from cell debris by centrifugation with $13,000 \times \mathrm{g}$ at $4^{\circ} \mathrm{C}$ for $30 \mathrm{~min}$ followed by plasmid DNA precipitation by addition of 0.8 volumes of 2-propanol. Plasmid DNA was collected by centrifugation, washed with $70 \%(\mathrm{v} / \mathrm{v})$ ethanol, dried, and reconstituted in 30-50 ( $\mathrm{L}$ sterile water. In cases in which highly pure plasmid DNA was required (e.g. for transfection of cultured mammalian cells), small, medium or large quantities of DNA were purified using the NucleoBond ${ }^{\circledR}$ Plasmid, PC 100, or PC 500 Kit from Macherey \& Nagel according to the manufacturer's instructions.

The concentration and purity of the DNA was assessed by measuring the OD at 260 and $280 \mathrm{~nm}$ using a Nanodrop spectrophotometer (Thermo Fischer Scientific). Only DNA with an $\mathrm{OD}_{260 / 280}$ ranging from 1.8 to 2.0 was used for mammalian cell transfection. 


\subsubsection{Cloning}

Restriction digestion of DNA by endonucleases

The enzymes and buffer system of Fermentas or New England Biolabs were used for DNA restrictions. Reaction conditions were chosen according to the manufacturer's instructions. For preparative restrictions, 1 /g DNA was digested in a volume of $50 \mathrm{C} \mathrm{L}$ in the presence of approximately 2-10 units of enzyme at $37^{\circ} \mathrm{C}$ for 2-4 h; analytical restrictions were carried out with 200-500 ng DNA in a volume of 20 / $\mathrm{L}$ using 3-5 units of enzyme for 1-2 h. To avoid star activity, the volume of the enzyme never exceeded $1 / 10$ of the reaction volume.

Agarose gel electrophoresis and isolation of DNA fragments

DNA fragments of different sizes were separated by agarose gel electrophoresis. Depending on the fragment size, $0.5-2 \%(w / v)$ agarose gels were used. DNA samples were supplemented with an appropriate amount of 6x DNA loading dye, were loaded on the gel, and were separated in TAE buffer at 80 Volt. For visualization, DNA was stained in a TAE bath containing $1 \mathrm{rg} / \mathrm{mL}$ ethidiumbromide and was detected with UV light of $365 \mathrm{~nm}$. Gel slices containing desired DNA fragments were excised and DNA was extracted using the NucleoSpin $^{\circledR}$ Extract II Kit from Macherey \& Nagel according to the manufacturers' instructions. DNA was typically eluted in $20-40 / \mathrm{L}$ of elution buffer.

\section{Ligation of DNA fragments}

Ligations were performed using 50-100 ng of vector in a molar vector to insert ratio ranging from $1: 3$ to $1: 9$. Reactions were performed in a total volume of 10 ( $L$ containing 1 Weiss unit T4 DNA ligase (Fermentas or NEB), ligation buffer and $1 \mathrm{mM}$ additional ATP. The ligation reaction was typically performed at $16^{\circ} \mathrm{C}$ over night and the entire mix was transformed into $\mathrm{DH} 5 \alpha$.

mRNA and cDNA preparation

mRNA was prepared from HeLa cells using the NucleoSpin ${ }^{\circledR}$ RNAll kit from Macherey \& Nagel according to the manufacturer's instructions. Isolated mRNA 
was then transcribed into cDNA using the First-strand cDNA Synthesis with RevertAid $^{\mathrm{TM}}$ kit from Fermentas.

Polymerase chain reaction (PCR)

The amplification of specific DNA fragments by employing 5'- and 3'-flanking primers was performed by PCR, which was originally established by (Mullis et al. 1986). Reactions were set up in a final volume of $50 \mu \mathrm{L}$ with $50-100 \mathrm{ng}$ template DNA or 1-2 $\mu \mathrm{L}$ cDNA, $20 \mathrm{pmol}$ of each forward and reverse primer, $200 \mu \mathrm{M}$ of each dNTP and 1 unit Phusion polymerase (Finnzymes). In case of GC-rich DNA, 3-5 \% (v/v) DMSO were included in the reaction. The annealing temperature for the appropriate oligonucleotide was calculated according to a web-based calculator (www.finnzymes.fi/tm determination.htm). Elongation times ranged from $15-30 \mathrm{sec} / \mathrm{kb}$. In general, amplification was performed with 35 cycles according the following program:

Initial denaturation

Denaturation during cycling

Annealing during cycling

Elongation during cycling

Final elongation $95^{\circ} \mathrm{C} \quad 1 \mathrm{~min}$

$\begin{array}{ll}95^{\circ} \mathrm{C} & 30 \mathrm{sec} \\ 45-72^{\circ} \mathrm{C} & 30 \mathrm{sec} \\ 72^{\circ} \mathrm{C} & 15-30 \mathrm{sec} / \mathrm{kb}\end{array}$

$72^{\circ} \mathrm{C}$

$5 \min$

$4^{\circ} \mathrm{C}$

Hold

\section{Site-directed mutagenesis}

Site-directed mutagenesis of plasmids was performed according to the QuickChange ${ }^{\circledR}$ Site-Directed Mutagenesis Protocol from Stratagene. Mutagenic primers were designed reverse-complementary to each other using the online tool PrimerX. (www.bioinformatics.org/primerx/). Typically, PCR reactions were performed in $50 \mu \mathrm{L}$ final volume containing 5-100 ng plasmid DNA, 5 pmol of forward and reverse primer, $5 \mathrm{nmol}$ of each dNTP, $2.5 \mu \mathrm{L}$ 10x Pfu buffer, and 1.5 units Pfu DNA polymerase (Promega). Amplification was performed in 28 cycles according to the following program: 
Initial denaturation

Denaturation during cycling

Annealing during cycling

Elongation during cycling

Final elongation $95^{\circ} \mathrm{C}$

$2 \min$

$95^{\circ} \mathrm{C} \quad 30 \mathrm{sec}$

$45-72^{\circ} \mathrm{C} \quad 1 \mathrm{~min}$

$72^{\circ} \mathrm{C}$

1.5 - $2 \mathrm{~min} / \mathrm{kb}$

$72^{\circ} \mathrm{C}$

$10 \min$

Subsequently the reaction was cooled to $4{ }^{\circ} \mathrm{C}$, incubated with 10 units Dpnl for 1 $\mathrm{h}$ at $37{ }^{\circ} \mathrm{C}$ for selective degradation of the methylated template DNA and transformed into competent bacteria.

\subsubsection{Sequencing of DNA}

All plasmids constructed via PCR amplification were verified by DNA sequencing carried out (on the basis of the chain termination method originally developed by Coulson and colleagues (Sanger, Nicklen, and Coulson 1977)) by the sequencing facility in the Department of Developmental Biochemistry, University of Göttingen or by GATC Biotech. For DNA plasmids sequenced by the facility in Göttingen, reactions were based on the BigDye Terminator v1.1 cycle sequencing kit (Applied Biosystems) and were setup according to the protocol given by the sequencing facility. Alternatively, 30 / $\mathrm{L}$ of $30-100 \mathrm{ng} / \mathrm{L}$ plasmid DNA and 10 $\mathrm{pmol} /$ / $\mathrm{L}$ primer were sent to GATC Biotech for sequencing. Sequences were analyzed using the software tools Vector NTI (Invitrogen) or SeqMan/SeqBuilder of the Lasergene Suite (DNAStar).

\subsubsection{Plasmids constructed in this work}

Human Fbxw5 plasmids

pCDNA3.1-flag-Fbxw5 was constructed by PCR amplification of Fbxw5 from HeLa cDNA (primers \#1249/1250) and subsequent cloning of the PCR product into pCDNA3.1-flag-USP25 (Meulmeester et al. 2008) using BamHI and Notl sites (thereby replacing the USP25). After verification of the sequence (primers \#1811/1257-61) Fbxw5 was subcloned into several vectors, again using BamHI 
and Notl sites. This way pCDNA3.1-HA-Fbxw5, pFASTBacHT-B-His-Fbxw5 pGEX-6P3-GST-Fbxw5, and pET28a-His-Fbxw5 were created. pGEX-4T1-Fbxw5-rbs-Skp1 $\Delta \Delta$ was generated by PCR amplification of Fbxw5 from pCDNA3.1-flag-Fbxw5 (primers \#1805/1806) followed by cloning of the PCR fragment into HindIII and Xhol sites of pGEX-4T1-Skp2 $\Delta \mathrm{N}$-rbs-Skp1 $\Delta \Delta$ (Li et al. 2005), thereby replacing Skp2 by Fbxw5.

To obtain pCDNA3.1-flag-Fbxw5 $\Delta \mathrm{F}-$ box $(\Delta \mathrm{N} 80)$, an Fbxw5 construct with an $\mathrm{N}$ terminal deletion lacking the F-box domain, nucleotides corresponding to aa 81566 were PCR amplified (primers \#1806/1820) from pCDNA3.1-flag-Fbxw5 and were cloned into BamHI and Xhol sites of pCDNA3.1-flag. For generation of pCDNA3.1-flag-Fbxw5R498A, site directed mutagenesis was performed on pCDNA3.1-flag-Fbxw5 (primers \#1814/1815).

\section{Mouse Fbxw5 plasmids}

pCDNA3.1-flag-m-Fbxw5 and pCDNA3.1-HA-m-Fbxw5 were constructed by swapping BamHI-Notl fragments encoding for $\mathrm{mFbxw} 5$ from pRSFDuet-His-MBPmFbxw5 to pCDNA3.1-flag and pCDNA3.1-HA, respectively.

To obtain pCDNA3.1-flag-m-Fbxw5 $\Delta \mathrm{F}-$ box $(\Delta \mathrm{N} 81)$ and pCDNA3.1-flag-mFbxw5-1-468, nucleotides corresponding to aa 82-573 (primers \#1893/1894) and aa 1-468 (primers \#1895/1896) were PCR amplified from pRSFDuet-HisMBP-mFbxw5 and cloned into BamHI and Notl sites of pCDNA3.1-flag.

pIRES-hrGFP1a-m-Fbxw5 and pIRES-hrGFP1a-m-Fbxw5 $\Delta \mathrm{F}-$ box( $\Delta$ N81) were cloned by PCR amplification of mFbxw5 from pCDNA3.1-flag-mFbxw5 (primers \#1920/1921) and mFbxw5 $\Delta$ N81 from pCDNA3.1-flag-mFbxw5 $\Delta$ N81 (primers \#1921/1945), respectively; and were subsequently cloned into Notl and BamHI sites of pIRES-hrGFP1a.

For mFbxw5 plasmids generated in PCR-based approaches, sequencing (primers \#1916-1918) was performed to assure the integrity of the insert.

Human Eps8 plasmids

For construction of pCDNA3.1-flag-h-Eps8, a DNA fragment encoding Eps8 was first amplified by PCR from HeLa cDNA (primers \#1866/1867) and was subsequently cloned into pCDNA3.1-flag using BamHI and Xhol sites. After 
verification of the sequence (primers \#1877-81), Eps8 was subcloned into pCDNA3.1-HA via BamHI and Xhol sites, creating pCDNA3.1-HA-h-Eps8. To generate pCDNA3.1-flag-h-Eps8K544R, site-directed mutagenesis was performed using pCDNA3.1-flag-hu-Eps8 as template (primers \#1882/1883).

Human Nek9 plasmids

To obtain pCDNA3.1-flag-Nek9 and pCDNA3.1-HA-Nek9, a nucleotide fragment encoding human Nek9 was PCR amplified from pDNR-LIB-hNek9 (\#1907/1919) followed by ligation of the Bglll-Notl fragment into BamHI and Notl sites of pCDNA3.1-flag and pCDNA3.1-HA, respectively. The identity of Nek9 within the generated plasmids was verified by sequencing (primers \#1912-1915).

\section{Ubiquitin plasmids}

To generate Ubiquitin plasmids for bacterial expression, Ubiquitin, UbiquitinK48R, and UbiquitinK29,48,63R were swapped from pCDNA3.1-HA to pET28a via EcoRI and Xhol sites. This way pET28a-His-Ubiquitin, pET28a-HisUbiquitinK48R, pET28a-His-UbiquitinK29,48,63R were created.

Ubiquitin E2 plasmids

pGEX-6P3-GST-UbcH5B and pGEX-6P3-GST-Cdc34 were generated by PCR of the respective sequence from pGEX-4X1-GST-UbcH5B (\#1809/1810) and pGEX4X1-GST-Cdc34 (primers \#1798/1799) followed by cloning of the inserts into pGEX-6P3 using BamHI and Xhol sites.

Plasmids for shRNA expression

pSUPER-puro-epi-nt and pSUPER-puro-epi-shFbxw5-1-4 were constructed by annealing cognate forward and reverse oligonucleotides (primers \#1933-1944) followed by cloning of the double stranded DNA (containing terminal BamHI and Hindlll sites) into Bglll and Hindlll sites of pSUPER-puro-epi. To minimize false positive clones, ligation reactions were digested with $\mathrm{BamHI}$ prior to transformation. 


\subsection{Biochemical methods}

\subsubsection{Measurement of protein concentration}

Protein concentrations of solutions and mammalian cell lysates were determined via the Pierce 660nm Assay (Pierce), according to the manufacturer's instructions. While the detailed assay chemistry is proprietary, the underlying mechanism is based on a proprietary dye-metal complex that binds to proteins in acidic conditions, causing a shift in the dye's absorption maximum, which is measured at $660 \mathrm{~nm}$. The dye-metal complex binds to proteins in a manner similar to Coomassie dye; however, unlike Coomassie-based assays (e.g. Bradford assay (Bradford 1976)), the Pierce $660 \mathrm{~nm}$ Protein Assay is fully compatible with a broad range of detergents.

Quantification of recombinant proteins was performed by comparison of defined amounts of BSA (Pierce) and increasing amounts of protein of interest on the same Coomassie -stained polyacrylamide gel.

\subsubsection{SDS PAGE and detection of proteins}

SDS polyacrylamide gel electrophoresis (SDS PAGE)

Separation of proteins was performed by SDS polyacrylamide gel electrophoresis according to the system described by Laemmli (Laemmli 1970). In most cases continuous $5-20 \%$ gradient gels or $8 \%$ gels were used. Gels were prepared in blocks of eight gels in casting blocks equipped with an inlet at the bottom and a rim at the central bottom of the casting block allowing to fill a batch of gels simultaneously. Equal volumes of $5 \%$ and $20 \%$ (w/v) polyacrylamide solutions in $0.4 \mathrm{M}$ Tris $-\mathrm{HCl} \mathrm{pH} 8.8,0.1 \%$ (w.v) SDS were prepared together fitting the casting block. Polymerization was started by adding APS and TEMED (each 0.06 $\%(\mathrm{w} / \mathrm{v}) /(\mathrm{v} / \mathrm{v})$ for the $5 \%$ solution and $0.05 \%(\mathrm{w} / \mathrm{v}) /(\mathrm{v} / \mathrm{v})$ for the $20 \%$ solution). The solutions were filled into the casting block using a double-cylindrical gradient mixer. This way, a continuous gradient of $5 \%$ at the top and $20 \%$ towards the bottom of the gels was generated. An overlay of 2-propanol assured an even surface. After polymerization of the separation gel, 2-propanol was removed and the stacking gel consisting of $4 \%(\mathrm{w} / \mathrm{v})$ polyacrylamide, $50 \mathrm{mM}$ Tris- $\mathrm{HCl} \mathrm{pH} 6.8$, 
$0.1 \%(\mathrm{w} / \mathrm{v})$ SDS, $0.001 \%(\mathrm{w} / \mathrm{v})$ APS and $0.001 \%(\mathrm{v} / \mathrm{v})$ TEMED was poured on top of the separation gel. After insertion of combs gels were allowed to polymerize for at least 2 hours. The gels were run with Laemmli buffer at $20 \mathrm{~mA} /$ $300 \mathrm{~V}$ per gel at room temperature. Before loading, samples were adjusted to approximately $50 \mathrm{mM}$ Tris- $\mathrm{HCl} \mathrm{pH} \mathrm{6.8,2 \% (w/v)} \mathrm{SDS,} 0.1 \%(\mathrm{w} / \mathrm{v})$ bromophenol blue, $10 \%$ glycerol, $100 \mathrm{mM}$ DTT with one-, two-, or four-fold concentrated SDS sample buffer and were boiled at $95^{\circ} \mathrm{C}$ for a few minutes.

\section{Coomassie staining}

For most applications, gels were stained for at least 1 hour in Coomassie-staining solution and then incubated in Coomassie-destaining solution until all excess dye was removed from the gel.

Gels for mass spectrometry analysis were stained with a different method, as described previously by (Neuhoff et al., 1985). In short, gels were fixed in a solution of $40 \%$ ethanol and $10 \%$ acetic acid for 20-30 minutes, the gels were rehydrated for some minutes in water and were stained in a solution of $1.6 \%$ ortho-phosphoric acid, $8 \%$ ammonium sulfate, $4 \%$ methanol and $0.08 \%$ Coomassie G-250. In this case, destaining of the gel was not necessary. For documentation gels were scanned via a 4990 Photo Scanner (Epson).

\section{Immunoblotting}

Prior to immunological detection, proteins from polyacrylamide gels were transferred onto nitrocellulose membranes after SDS PAGE using a semi-dry western blot apparatus (Kyhse-Andersen 1984). The gel was mounted between Whatman paper stacks soaked in western blot buffer (25 mM Tris, $193 \mathrm{mM}$ glycine, $20 \%(\mathrm{v} / \mathrm{v})$ methanol, $0.036 \%(\mathrm{w} / \mathrm{v}) \mathrm{SDS})$ and proteins were transferred onto the membrane at $200 \mathrm{~mA} / 300 \mathrm{~V}$ for $2 \mathrm{~h}$. The transfer was controlled by staining protein with $0.5 \%(\mathrm{w} / \mathrm{v})$ Ponceau $S$ in $1 \%(\mathrm{v} / \mathrm{v})$ acetic acid and excess dye was removed by washing with $1 \%$ acetic acid. Unspecific binding sites on the membrane were blocked by incubation with blocking buffer (5\% (w/v) skim milk (AppliChem) in PBST) for at least 60 minutes. For immunological detection of proteins, primary antibodies were applied diluted in blocking buffer for at least $2 \mathrm{~h}$ at room temperature or overnight at $4{ }^{\circ} \mathrm{C}$ followed by extensive washing steps 
with at least three changes of PBST. Secondary horseradish peroxidase coupled antibodies were then applied for $1.5 \mathrm{~h}$ at room temperature. After removal of unbound antibodies by extensive washing with PBST, bound antibodies were detected by chemiluminescence using ECL kits from Pierce (Pierce ECL Western Blotting Substrate) or Millipore (Immobilon Western Chemiluminescent HRP Substrate); exposed films were developed using an automatic developing machine. In case a single membrane was used to detect several proteins, the membrane was either cut in stripes to separate specific molecular weight ranges for individual immunostaining or proteins were consecutively detected. For the latter approach, old signals were quenched by irreversibly inhibiting the horseradish peroxidase already present on the membrane by addition of $5 \mathrm{mM}$ sodium azide to the following primary antibody.

\subsubsection{Production of recombinant proteins in bacteria}

\section{Protein expression}

For protein expression in bacteria, suitable strains were transformed with the plasmid encoding for the gene of interest followed by direct inoculation of LB with pre-cultures. Supplementation of LB with appropriate antibiotics ensured the selection of positively transformed cells. Pre-cultures were grown over night at $37^{\circ} \mathrm{C}$, cells were harvested by centrifugation and subsequently diluted $1: 100$ into fresh LB medium containing antibiotics to maintain the selection pressure. Recombinant protein production was induced by IPTG.

\section{Expression and purification of GST-Prescission}

GST-PreScission was purified following standard procedures. The production of GST-tagged Prescission protease in BL21(DE3) was induced by $1 \mathrm{mM} \mathrm{IPTG}$ at an $\mathrm{OD}_{600}$ of 0.8 . Cells were then inbcubated at $37^{\circ} \mathrm{C}$ for $5 \mathrm{~h}$ and harvested by centrifugation. Cell pellets were resuspended in $35 \mathrm{~mL}$ Prescission buffer A (PBS $\mathrm{pH}$ 7.4, $2.5 \mathrm{mM} \mathrm{MgCl}$, 2 mM DTT, 2 mM EDTA, aprotinin, leupeptin, pepstatin) per liter culture and subjected to lysis by EmulsionFlex. After clearing of the lysate by centrifugation with $100,000 \times \mathrm{xg}$ at $4^{\circ} \mathrm{C}$ for $1 \mathrm{~h}$, the supernatant fraction was incubated with $5 \mathrm{~mL}$ glutathione sepharose (Fast Flow 4B, GE Healthcare) per 
liter culture under constant rotation at $4^{\circ} \mathrm{C}$ for $2 \mathrm{~h}$. The beads were washed with Prescission buffer A followed by several washes with Prescission buffer B (50 $\mathrm{mM}$ Tris $\mathrm{pH}$ 7.9, $100 \mathrm{mM} \mathrm{NaCl}, 2.5 \mathrm{mM} \mathrm{MgCl}$, $2 \mathrm{mM}$ DTT, $2 \mathrm{mM}$ EDTA, aprotinin, leupeptin, pepstatin). GST-Prescission was eluted from the beads using Prescission buffer B supplemented with $20 \mathrm{mM}$ reduced glutathione and was further purified by molecular sieving over a preparative Superdex 75 in $20 \mathrm{mM}$ Tris pH 7.5, $100 \mathrm{mM} \mathrm{NaCl}, 2 \mathrm{mM}$ DTT. Pure GST-Prescission was concentrated by centrifuge concentrators and supplemented with $20 \%$ glycerol and $2 \mathrm{mM} \beta$ mercaptoethanol before aliquoting and storing at $-20^{\circ} \mathrm{C}$.

Average yield: $\quad 8 \mathrm{mg} / 1 \mathrm{~L}$ culture

Activity:

Active in cleaving various GST-PreS-tagged proteins (see below)

\section{Expression and purification of His-TEV}

His-TEV was purified following standard procedures. The production of Histagged TEV protease in BL21(DE3) was induced by $0.5 \mathrm{mM} \mathrm{IPTG}$ at an $\mathrm{OD}_{600}$ of 0.8 . After incubation at $30^{\circ} \mathrm{C}$ for $2 \mathrm{~h}$, cells were harvested by centrifugation and resuspended in $25 \mathrm{~mL}$ TEV buffer ( $20 \mathrm{mM}$ Tris $\mathrm{pH}$ 7.5, $100 \mathrm{mM} \mathrm{NaCl}, 2 \mathrm{mM} \beta$ mercaptoethanol, aprotinin, leupeptin, pepstatin) per liter culture. After clearing of the lysate by centrifugation with $100,000 x g$ at $4^{\circ} \mathrm{C}$ for $1 \mathrm{~h}$, the supernatant fraction was passed over $5 \mathrm{~mL}$ Ni-NTA agarose (Qiagen) per liter culture. The $\mathrm{Ni}^{2+}$ beads were washed with TEV buffer supplemented with increasing amounts of imidazole $(10,25$ and $50 \mathrm{mM})$ and His-TEV was eluted using TEV buffer containing $250 \mathrm{mM}$ imidazole. Elution fractions were analyzed by SDS PAGE and Coomassie staining and purest fractions were pooled and were $3 x$ dialyzed against TEV buffer at $4^{\circ} \mathrm{C}$. Finally, pure His-TEV was concentrated by centrifuge concentrators and supplemented with $50 \%$ glycerol before aliquoting, flash-freezing in liquid nitrogen, and storing at $-80^{\circ} \mathrm{C}$.

Average yield: $\quad 3 \mathrm{mg} / 1 \mathrm{~L}$ culture

Activity:

Active in cleaving His-TEV-tagged and GST-TEV-tagged proteins (see below) 
Expression and purification of His-Ubiquitin and His-Ubiquitin mutants

His-Ubiquitin and His-Ubiquitin mutant production in BL21(DE3) pLysS was induced by $0.4 \mathrm{mM} \mathrm{IPTG}$ at an $\mathrm{OD}_{600}$ of 0.6 . Cells were then grown at $37^{\circ} \mathrm{C}$ for $4 \mathrm{~h}$ and harvested by centrifugation. Cell pellets were resuspended in $25 \mathrm{~mL}$ Ubiquitin buffer A (50 mM Tris pH 7.6, $10 \mathrm{mM} \mathrm{MgCl}, 2 \mathrm{mM} \beta$-mercaptoethanol, aprotinin, leupeptin, pepstatin, PMSF) per liter culture and subjected to lysis by EmulsionFlex. After clearing of the lysate by centrifugation with $100,000 \mathrm{xg}$ at $4^{\circ} \mathrm{C}$ for $1 \mathrm{~h}$, the supernatant fraction was incubated together with $5 \mathrm{~mL} \mathrm{Ni-NTA}$ agarose (Qiagen) per liter culture under constant rotation at $4{ }^{\circ} \mathrm{C}$ for $2 \mathrm{~h}$. The $\mathrm{Ni}^{2+}$ beads were washed extensively with Ubiquitin buffer $B(50 \mathrm{mM}$ sodium phosphate buffer $\mathrm{pH} 6.3,100 \mathrm{mM} \mathrm{NaCl}, 10 \mathrm{mM}$ imidazole $2 \mathrm{mM} \beta$ mercaptoethanol, aprotinin, leupeptin, pepstatin, PMSF). His-Ubiquitin was eluted from the beads using Ubiquitin buffer $\mathrm{B}$ with a $\mathrm{pH}$ of 8.0 and $250 \mathrm{mM}$ imidazole, was $3 x$ dialyzed against Ubiquitin buffer $\mathrm{C}(50 \mathrm{mM}$ ammonium acetate $\mathrm{pH} 4.5$, $1 \mathrm{mM}$ DTT), and was bound to a SP-sepharose (Sigma) column (10mL per liter of culture). After washing of the beads with Ubiquitin buffer $\mathrm{C}$, His-Ubiquitin was eluted from the beads using Ubiquitin buffer $C$ supplemented with $1 \mathrm{M} \mathrm{NaCl}$ followed by buffer exchange to TB by repeated concentration and dilution using a centrifuge concentrator. Purified Ubiquitin fractions were aliquoted, flash-frozen, and stored at $-80^{\circ} \mathrm{C}$.

Average yield: $\quad 25-30 \mathrm{mg} / 1 \mathrm{~L}$ culture

\section{Expression and purification of Ube1-His}

Purification of C-terminally His-tagged human Ube1 was preformed as previously described (Pickart and Raasi 2005). In short, Ube1-His production in BL21(DE3) pLysS was induced by $0.4 \mathrm{mM} \mathrm{IPTG}$ at an $\mathrm{OD}_{600}$ of 0.8 . Cells were then grown at $16^{\circ} \mathrm{C}$ for $4 \mathrm{~h}$ and harvested by centrifugation. Ube1-His was then purified in a three-step protocol including $\mathrm{Ni}^{2+}$ affinity, anion exchange (MonoQ), and gel filtration (S200) chromatography and was stored in $50 \mathrm{mM}$ Tris $\mathrm{pH} 8.0,100 \mathrm{mM}$ $\mathrm{NaCl}, 5 \mathrm{mM}$ DTT in small aliquots at $-80^{\circ} \mathrm{C}$. 
Average yield: $\quad 0.5-1 \mathrm{mg} / 1 \mathrm{~L}$ culture

\section{Expression and purification of $\mathrm{UbcH} 5 \mathrm{~B}$ and $\mathrm{Cdc} 34$}

The production of GST-tagged UbcH5B and Cdc34 in BL21(DE3) pLysS was induced by $0.5 \mathrm{mM} \mathrm{IPTG}$ at an $\mathrm{OD}_{600}$ of 0.6 . After incubation at $30^{\circ} \mathrm{C}$ for $4 \mathrm{~h}$, cells were harvested by centrifugation and resuspended in $25 \mathrm{~mL}$ E2 buffer A $(50 \mathrm{mM}$ Tris $\mathrm{pH} 8.0,300 \mathrm{mM} \mathrm{NaCl}, 1 \mathrm{mM} \beta$-mercaptoethanol, aprotinin, leupeptin, pepstatin, PMSF) per $1 \mathrm{~L}$ culture. Cells were lyzed with the EmulsionFlex and lysates cleared from debris by centrifugation with $100,000 \mathrm{xg}$ at $4^{\circ} \mathrm{C}$ for $1 \mathrm{~h}$. Lysates were then incubated with $2 \mathrm{~mL}$ glutathione sepharose (Fast Flow 4B, GE Healthcare) per liter culture under constant rotation at $4^{\circ} \mathrm{C}$ for $2 \mathrm{~h}$. The beads were then washed with E2 buffer A, followed by elution of GST-UbcH5B/Cdc34 using $50 \mathrm{mM}$ Tris $\mathrm{pH}$ 8.0, $150 \mathrm{mM} \mathrm{NaCl}, 1 \mathrm{mM}$ EDTA, $1 \mathrm{mM} \beta$-mercaptoethanol, $25 \mathrm{mM}$ glutathione. For GST-tag cleavage, the concentration of GST-UbcH5B/Cdc34 was roughly estimated via SDS PAGE and Coomassie staining and for each 100 $\mu \mathrm{g}$ of fusion protein, $0.5-1 \mu \mathrm{g}$ of Precission protease was added. The reaction mixture was then incubated at $4^{\circ} \mathrm{C}$ for $12-16 \mathrm{~h}$, followed by separation of untagged $\mathrm{UbcH} 5 \mathrm{c} / \mathrm{Cdc} 34$ from free GST and GST-Prescission via gel filtration chromatography over a preparative Superdex 75 column in $50 \mathrm{mM}$ Tris $\mathrm{pH} 8.0$, $100 \mathrm{mM} \mathrm{NaCl}, 5 \mathrm{mM}$ DTT. Purified UbcH5B/Cdc34 fractions were aliquoted, flash-frozen, and stored at $-80^{\circ} \mathrm{C}$.

Average yield: $\quad 1-2 \mathrm{mg} / 1 \mathrm{~L}$ culture

\section{Expression of human Fbxw5 in bacteria}

In the course of this thesis, it was not possible to purify soluble human Fbxw5 protein from bacteria in significant amounts, although several parameters for expression and purification were tested:

Bacterial strains: $\quad$ BL21(DE3), BL21(DE3) pLysS or pLysE, Rosetta, Rosetta2, Arctic Express ${ }^{\mathrm{TM}}$ (DE3) RIL

Expression temperatures: $12^{\circ} \mathrm{C}, 16^{\circ} \mathrm{C}$, or $37^{\circ} \mathrm{C}$

IPTG concentrations: $\quad 0.1,0.5$, or $1 \mathrm{mM}$ 
Expression tags:

Co-expression:

Lysis buffer:
GST, His, untagged

GST-Skp1 $1 \Delta \Delta$ (via bicistronic expression vector)

High and low salt concentrations, presence and absence of detergent

In addition, purification of GST- and His-Fbxw5 under denaturing conditions from inclusion bodies with subsequent re-folding (as previously described for RanGAP1 (Mahajan et al. 1997)) did also not result in soluble protein; however, this approach yielded large amounts of highly pure, unfolded GST- and HisFbxw5 that could be used for antibody production.

\section{Purification of denatured His- and GST-tagged Fbxw5 from inclusion bodies}

His-/GST-tagged Fbxw5 was purified according to a modified version of the previously described protocol for RanGAP1 purification (Mahajan et al. 1997). In short, His- and GST-Fbxw5 production in BL21(DE3) pLysS was induced by 1 $\mathrm{mM}$ IPTG at an $\mathrm{OD}_{600}$ of 1.0 . Cells were then grown at $30^{\circ} \mathrm{C}$ for $4 \mathrm{~h}$ and harvested by centrifugation. After resuspension of cells in $25 \mathrm{~mL}$ buffer A $(50 \mathrm{mM}$ Tris $\mathrm{pH}$ 8.0, $100 \mathrm{mM} \mathrm{NaCl}, 2 \mathrm{mM}$ DTT, $2 \mathrm{mM}$ EDTA, aprotinin, leupeptin, pepstatin, PMSF) per liter culture, cells were lyzed via the Emulsionflex. The bacterial pellet was then washed by repeated centrifugation and careful resuspension steps using buffer $A$ containing $2 \%$ Trition, buffer $A$ containing $2 \mathrm{M}$ Urea, buffer $\mathrm{A}$ containing $4 \mathrm{M}$ Urea, and buffer $A$ containing $6 \mathrm{M}$ Urea. For these resuspension steps, a tight fitting glass douncer was employed. His-/GST-Fbxw5 was subsequently extracted from the bacterial pellet using UT buffer $\mathrm{pH} 8.0$ and was bound to $3 \mathrm{~mL}$ SP Sepharose (Sigma) per liter culture. Beads were extensively washed with UT buffer pH 8.0 before elution of His-/GST-Fbxw5 using UT buffer $\mathrm{pH} 7.4$ containing $1 \mathrm{M} \mathrm{NaCl}$ and three times dialysis of the eluate against PBS. His-/GST-Fbxw5 (which precipitates from solution during dialysis) was collected by centrifugation and precipitates were air-dried. Finally, the proteins were either solubilized in UT buffer or resuspended in PBS, flash-frozen, and stored at $-80^{\circ} \mathrm{C}$.

Average yield: $\quad 6-8 \mathrm{mg}$ precipitated protein / $1 \mathrm{~L}$ culture 


\section{Expression and purification of soluble mFbxw5/Skp1 complex}

To obtain significant amounts of soluble mouse protein in bacteria, mFbxw5 had to be co-expressed with its interaction partner Skp1. Production of His-MBPmFbxw5 and GST-Skp1 in BL21(DE3) was induced by 0.5 mM IPTG at an OD 600 of 0.8 . Cells were then grown at $16^{\circ} \mathrm{C}$ over night, harvested by centrifugation and resuspended in $20 \mathrm{~mL}$ mFbxw5 Buffer $\mathrm{A}(50 \mathrm{mM}$ Tris $\mathrm{pH} 8.0,300 \mathrm{mM} \mathrm{NaCl}, 5$ mM $\beta$-mercaptoethanol, $10 \mathrm{mM}$ imidazole, aprotinin, leupeptin, pepstatin, PMSF) per $1 \mathrm{~L}$ culture. Cells were lyzed with the EmulsionFlex and the lysate was cleared from debris by centrifugation with $100,000 x g$ at $4^{\circ} \mathrm{C}$ for $1 \mathrm{~h}$. After passage of the lysate over $1 \mathrm{~mL}$ Ni-NTA agarose (Qiagen) per liter culture and washing of the $\mathrm{Ni}^{2+}$ beads with mFbxw5 Buffer A containing $20 \mathrm{mM}$ imidazole, the His-MPBmFbxw5/GST-Skp1 complex was eluted with mFbxw5 buffer A containing 250 $\mathrm{mM}$ imidazole. The complex was further purified by molecular sieving over a preparative Superdex 200 column in mFbxw5 Buffer B (50 mM Tris pH 8.0, 100 $\mathrm{mM} \mathrm{NaCl}, 5 \mathrm{mM} \beta$-mercaptoethanol) and its concentration was subsequently estimated via SDS PAGE and Coomassie staining. For each $100 \mu \mathrm{g}$ of fusion protein, $0.5-1 \mu \mathrm{g}$ of TEV protease was added, followed by incubation at $4^{\circ} \mathrm{C}$ for 12-16h to allow for efficient His-MBP and GST-tag cleavage. Separation of untagged mFbxw5/Skp1 complex from tags and TEV protease was achieved by a second run over a preparative Superdex 200 column in mFbxw5 Buffer B. mFbxw5/Skp1 fractions were concentrated using a centrifuge concentrator, aliquoted, flash-frozen in liquid nitrogen, and stored at $-80^{\circ} \mathrm{C}$.

Average yield: $\quad 0.2-0.4 \mathrm{mg} / 12 \mathrm{~L}$ culture

\subsubsection{Production of recombinant proteins in insect cells}

Human Fbxw5 from bacteria seemed to be heavily insoluble under various tested conditions. Therefore, baculovirus-assisted insect cell expression was employed to provide the eukaryotic folding machinery for Fbxw5 production. 
Protein expression in insect cells via the baculovirus system requires a recombinant virus. Therefore, human Fbxw5 baculovirus was generated following the instructions of the Bac-to-Bac ${ }^{\circledR}$ Baculovirus Expression system (Invitrogen, (Luckow et al. 1993)). In short, the pFASTBacHT-His-Fbxw5 donor plasmid was transformed into DH10Bac competent cells, which contain the bacmid with a miniattTn7 target site and the helper plasmid. The mini Tn7 element (containing the sequence of human Fbxw5) on the pFASTBacHT donor plasmid could transpose to the mini-attTn7 site on the bacmid in the presence of transposition proteins provided by the helper plasmid. Colonies containing recombinant human Fbxw5 bacmids were distinguished from those containing unaltered bacmids by bluewhite screening (insertion of the mini $\operatorname{Tn} 7$ element into the bacmid disrupts the lacZ $\alpha$ gene). High molecular weight DNA was then isolated from positive clones via mini prep and the insertion of the Fbxw5 sequence verified by sequencing (primers \#1258/1863). To obtain recombinant human Fbxw5 baculovirus, Fbxw5 bacmids were transfected into SF9 cells with Cellfectinll ${ }^{\circledR}$ Reagent according to the manufacturer's guidelines. $72 \mathrm{~h}$ post transfection cells showed signs of late viral infection, indicating virus budding and release into the media. The medium of the infected cells (P1 viral stock) was harvested and cleared from cell debris via centrifugation. For virus titer amplification, P2 and P3 viral stocks were generated by using the P1 (or P2) viral stock in a 1:10 dilution for infection of SF9 cells for $72 \mathrm{~h}$ followed by collection of the medium as described above. Viral stocks were stored protected from light at $4^{\circ} \mathrm{C}$ (for short term storage) or flash frozen in liquid nitrogen and stored at $-80^{\circ} \mathrm{C}$ (for long term storage).

\section{Expression and purification of His- $h F b x w 5 / S k p 1 \Delta \Delta$ complex}

The purification procedure of His-hFbxw5/Skp1 $\Delta \Delta$ complex was based on a protocol previously described for the Skp2/Skp1 complex (Li et al. 2005). Skp1 $\Delta \Delta$ refers to a human Skp1 version with two internal deletions, which are in the loops spanning residues 38 to 43 and 71 to 82 . These two internal deletions have no influence on its functional properties.

In short, SF9 cells were co-infected with high titer (P4) human Fbxw5 and Skp1 $\Delta \Delta$ viral stocks (the latter obtained from Brenda Schulman) for $48 \mathrm{~h}$ and harvested by centrifugation, The cell pellet was re-suspended in $50 \mathrm{~mL}$ hFbxw5 
buffer A (20 mM HEPES pH 7.8, $200 \mathrm{mM} \mathrm{NaCl}, 5 \mathrm{mM} \beta$-mercaptoethanol, 0.1\% NP-40, 5mM imidazole, aprotinin, leupeptin, pepstatin, PMSF) per liter of culture and cells were lysed by freezing in liquid nitrogen and thawing. The lysate was cleared from cell debris via centrifugation with $100,000 \times \mathrm{xg}$ at $4^{\circ} \mathrm{C}$ for $1 \mathrm{~h}$ and incubated with $4 \mathrm{~mL}$ Ni-NTA agarose (Qiagen) per liter culture under constant rotation at $4^{\circ} \mathrm{C}$ for $2 \mathrm{~h}$. The $\mathrm{Ni}^{2+}$ beads were washed with hFbxw5 Buffer containing $20 \mathrm{mM}$ imidazole followed by elution of the His-hFbxw5/Skp1 $\Delta \Delta$ complex with hFbxw5 buffer containing $200 \mathrm{mM}$ imidazole. The complex was further purified by molecular sieving over a preparative Superdex 200 column in mFbxw5 buffer A devoid of imidazole, and protein containing fractions aliquoted, flash-frozen in liquid nitrogen, and stored at $-80^{\circ} \mathrm{C}$.

Average yield: $\quad 0.5 \mathrm{mg} / 1 \mathrm{~L}$ culture

\subsubsection{Production of recombinant proteins in mammalian cells}

\section{Expression and purification of flag-Fbxw5}

To obtain Fbxw5-containing E3 ligase complexes, flag-Fbxw5 was purified from HEK 293T cells via anti-flag immmunoprecipitation. Routinely, five $15 \mathrm{~cm}$ dishes of HEK 293T cells stably or transiently expressing flag-Fbxw5 were trypsinized, were washed twice with PBS containing $10 \mathrm{mM}$ NEM, and were resuspendend in two pellet volumes of flag-Fbxw5 buffer (TB buffer supplemented with $0.2 \%$ Tween20, 10 mM NEM, 5 mM EDTA, 5 mM EGTA, aprotinin, leupeptin, pepstatin, PMSF). Cells were allowed to lyse on ice for $20 \mathrm{~min}$ and lysates were then cleared from debris by centrifugation with $100,000 \times \mathrm{xg}$ at $4^{\circ} \mathrm{C}$ for $1 \mathrm{~h}$. The supernatant fraction was further pre-cleared by incubation with Protein $G$ beads under constant rotation for $30 \mathrm{~min}$ at $4^{\circ} \mathrm{C}$. After removal of Protein $\mathrm{G}$ beads by centrifugation, the lysate was incubated with $20 \mu \mathrm{L}$ ANTI-FLAG-M2 agarose (Sigma) under constant rotation for $2 \mathrm{~h}$ at $4^{\circ} \mathrm{C}$. The beads were quantitatively transferred into a new tube and washed with $3 \times 1 \mathrm{~mL}$ flag-Fbxw5 buffer. Depending on the application flag-Fbxw5 was intended for, different peptide elution protocols were employed. For mass spectrometry and immunoblotting analysis, flag-Fbxw5 was eluted from the antibodies with $3 \times 50 \mu \mathrm{L}$ flag-Fbxw5 
buffer containing $0.5 \mathrm{mg} / \mathrm{mL}$ flag peptide. Each elution step was carried out under vigorous shaking at $28^{\circ} \mathrm{C}$ for $15 \mathrm{~min}$ in a thermomixer (Eppendorf). Elution fractions were pooled, centrifuged, and transferred to a new tube to assure the absence of any beads in the eluate. In case flag-Fbxw5 immunoprecipitates were to be used in in vitro ubiquitylation assays, the elution procedure was altered in the following way: beads were washed $2 x$ with $S A B$ buffer prior to elution, which was carried out in SAB buffer supplemented with $0.5 \mathrm{mg} / \mathrm{mL}$ flag peptide.

Flag-Fbxw5 $\Delta$ N80 (lacking the N-terminal F-box domain) was purified following the same procedure as here described for the full-length version.

\section{Expression and purification of HA-Nek9}

Nek9 activity was reported to be detergent-sensitive (Holland et al. 2002). Taking this observation into account, a purification protocol similar to that described for flag-Fbxw5 was established. HA-Nek9 was purified from 293T cells via anti-HA immmunoprecipitation. Routinely, three $15 \mathrm{~cm}$ dishes of 293T cells transiently expressing HA-Nek9 were trypsinized, were washed twice with PBS, and were resuspendend in two pellet volumes of $50 \mathrm{mM}$ HEPES pH 7.5, $100 \mathrm{mM} \mathrm{NaCl}, 10$ $\mathrm{mM} \mathrm{MnCl}_{2}, 5 \mathrm{mM} \mathrm{MgCl}$, 2 mM EDTA, $3 \mathrm{mM}$ EGTA, $1 \mathrm{mM}$ DTT, 0.1\% NP-40 (a mild non-ionic detergent), aprotinin, leupeptin, pepstatin, PMSF, PhosSTOP (Roche). Cell lysis and pre-clearing were conducted as described for the flagFbxw5 purification. $20 \mu \mathrm{L} \alpha-\mathrm{HA}$ agarose (Sigma) were added to the pre-cleared lysate followed by incubation under constant rotation for $2 \mathrm{~h}$ at $4^{\circ} \mathrm{C}$. The beads were quantitatively transferred into a new tube and washed with $3 \times 1 \mathrm{~mL}$ Nek9 buffer (50 mM Tris pH 7.4, $150 \mathrm{mM} \mathrm{NaCl}, 10 \mathrm{mM} \mathrm{MnCl}$, $5 \mathrm{mM} \mathrm{MgCl}, 1 \mathrm{mM}$ DTT, aprotinin, leupeptin, pepstatin, PMSF, PhosSTOP (Roche)). HA-Nek9 was eluted from the antibodies with $3 \times 50 \mu \mathrm{L}$ Nek9 buffer containing $0.5 \mathrm{mg} / \mathrm{mL} \mathrm{HA}$ peptide. Each elution step was carried out under vigorous shaking at $28^{\circ} \mathrm{C}$ for 15 min in a thermomixer (Eppendorf). Elution fractions were pooled, centrifuged, and transferred to a new tube to assure the absence of any beads in the eluates. HANek9 immunoprecipitates were then aliquoted, flash frozen in liquid nitrogen, and stored at $-80^{\circ} \mathrm{C}$. 


\subsubsection{In vitro reconstitution of $\mathrm{SCF}^{\mathrm{Fbxw5}}$}

For in vitro reconstitution of $\mathrm{SCF}^{\mathrm{Fbxw5}}$, either $\mathrm{mFbxw5/Skp1}$ or HishFbxw5/Skp1 $\Delta \Delta$ complex and Nedd8 Cul1/Rbx1 complex (purified by Brenda Schulman) were carefully mixed in equimolar amounts and incubated on ice for $20 \mathrm{~min}$.

\subsubsection{In vitro phosphorylation and dephosphorylation reactions}

In vitro dephosphorylation of Eps8 with Calf Intestine Phosphatase (CIP)

For in vitro dephosphorylation, $5 \mu \mathrm{M}$ mouse full length His-Eps8 (obtained from Giorgio Scita and purified from 293T cells (Disanza et al. 2006) or SF9 cells (Disanza et al. 2004)) were incubated with $10 \mathrm{U}$ CIP (NEB) in buffer 3 (NEB) at $37^{\circ} \mathrm{C}$ for $1 \mathrm{~h}$. A reaction mix devoid of CIP was used as a negative control. To inactivate phoshphatase activity, $0.2 \mathrm{M}$ sodium orthovanadate were added. This protocol allows for quantitative dephosphorylation of Eps8 as judged by the faster migration behavior of CIP-treated Eps8 compared to untreated Eps8 in SDS PAGE.

In vitro phosphorylation reactions with HA-Nek9

In vitro kinase assays with immunopurified HA-Nek9 were performed in SAB buffer. $2.5 \mu \mathrm{M}$ mouse full length His-Eps8 (+/- prior CIP-treatment) or $2.5 \mu \mathrm{M}$ mFbxw5/Skp1 complex were incubated with different amounts of HA-Nek9 in the absence and presence of $5 \mathrm{mM} \mathrm{ATP}$ at $30^{\circ} \mathrm{C}$ for $60-90 \mathrm{~min}$.

\subsubsection{In vitro ubiquitylation reactions with recombinant proteins}

In vitro ubiquitylation were performed along general lines as described in (Deshaies 2005). Typically, reactions were performed in SAB buffer in 10-20 $\mu \mathrm{L}$ scale at $30^{\circ} \mathrm{C}$ for $90 \mathrm{~min}$ and were stopped by addition of 1 reaction volume of $2 \mathrm{x}$ SDS sample buffer, unless indicated otherwise. If possible, reactions were set up using stock mixes. Samples were analyzed by SDS PAGE followed by immunoblotting against the protein of interest. 
In vitro ubiquitylation with flag-Fbxw5 and flag-Fbxw5 4 N80

To test for Fbxw5-dependent ubiquitylation, $75 \mu \mathrm{M}$ His-Ubiquitin, $170 \mathrm{nM}$ Ube1His, $1 \mu \mathrm{M} \mathrm{UbcH5c}$ or $\mathrm{Cdc} 34$, and $5 \mathrm{mM}$ ATP were incubated together with different candidate and control proteins (e.g. $0.1 \mu \mathrm{M}$ His-Eps8, $1.25 \mu \mathrm{M}$ SUMO1 $\triangle \mathrm{C} 4$, or $0.1 \mu \mathrm{M}$ RanGAP1) in the absence and presence of increasing amounts of flag-Fbxw5 and flagFbxw5 $5 \mathrm{~N} 80$ immunoprecipitates.

In vitro ubiquitylation with $\mathrm{SCF}^{\mathrm{Fbxw5}}$

To test for $\mathrm{SCF}^{\mathrm{Fbxw5}}$-dependent ubiquitylation, $0.1 \mu \mathrm{M}$ His-Eps8 were incubated with $75 \mu \mathrm{M}$ His-Ubiquitin, $170 \mathrm{nM}$ Ube1-His, $1 \mu \mathrm{M} \mathrm{UbcH5c}$ or Cdc34, and $5 \mathrm{mM}$ ATP in the absence or presence of $150 \mathrm{nM}$ reconstituted $\mathrm{SCF}^{\text {hFbxw5 }}$ or SCF ${ }^{\mathrm{mFbxw}}$.

In vitro ubiqutiylation of CIP- and Nek9-treated Eps8

To analyze ubiquitylation properties of Eps8 pools with different phosphorylation states, $0.1 \mu \mathrm{M}$ CIP- and/or Nek9-treated His-Eps8 were incubated together with $75 \mu \mathrm{M}$ His-Ubiquitin, $170 \mathrm{nM}$ Ube1-His, $1 \mu \mathrm{M}$ UbcH5c, $150 \mathrm{nM} \mathrm{SCF}{ }^{\mathrm{mFbxw} 5}$, and 5 mM ATP for 30 and $60 \mathrm{~min}$.

2.2.9. Production and affinity purification of rabbit polyclonal Fbxw5 antibodies

Production of rabbit polyclonal Fbxw5 antibodies

Rabbit sera containing polyclonal Fbxw5 antibodies were produced commercially by Charles River Laboratories. Briefly, specific pathogen free (SFP) rabbits (strain NZW) were immunized according to a standard protocol (4 injections on days 0 , 28, 42 and 56). Per injection, $100 \mu \mathrm{g}$ denatured GST-Fbxw5 in 8M urea pH 8.0 and $100 \mu \mathrm{g}$ denatured GST-Fbxw5 in PBS both emulsified in Complete Freund's Adjuvant $\left(1^{\text {st }}\right.$ injection $)$ or emulsified in Incomplete Freund's Adjuvant $\left(2^{\text {nd }}, 3^{\text {rd }}\right.$, and $4^{\text {th }}$ injection) were used. Rabbits were sacrificed on day 70. Fbxw5 antibodies were purified from the serum of the final bleed. 
Affinity purification of Fbxw5 antibodies

Rabbit polyclonal Fbxw5 antibodies were affinity-purified from serum by adsorption to recombinant His-Fbxw5 immobilized on stripes followed by acid elution. Typically, $600 \mu \mathrm{g}$ His-Fbxw5 were separated via SDS PAGE, blotted on nitrocellulose, and stripes containing His-Fbxw5 were cut out. Stripes were incubated in blocking buffer for at least $30 \mathrm{~min}$ followed by washes with $0.2 \mathrm{M}$ acetic acid pH 2.7, $500 \mathrm{mM} \mathrm{NaCl}$ and PBS. For binding of Fbxw5 antibodies, HisFbxw5 stripes were incubated with a mixture of $10 \mathrm{~mL}$ rabbit serum and $10 \mathrm{~mL}$ PBS at $4^{\circ} \mathrm{C}$ over night. After extensive washing of the stripes with PBS containing $500 \mathrm{mM} \mathrm{NaCl}$, Fbxw5 antibodies were eluted with $0.2 \mathrm{M}$ acetic acid pH 2.7, 500 $\mathrm{mM} \mathrm{NaCl}$. Each $1 \mathrm{~mL}$ elution fraction was repeatedly pipetted over the stripes and immediately neutralized by addition of $200 \mu \mathrm{L} \quad$ M Tris Base. Antibodycontaining fractions were combined and concentrated to approximately 1-2 $\mathrm{mg} / \mathrm{ml}$. The buffer was changed to PBS, antibodies were mixed with 1 volume of $87 \%(\mathrm{v} / \mathrm{v})$ glycerol and stored in aliquots at $-20^{\circ} \mathrm{C}$.

Average yield: $\quad 0.8 \mathrm{mg} / 10 \mathrm{~mL}$ serum

Western Blot:

recognize up to $0.1 \mathrm{ng}$ of recombinant Fbxw5; recognize endogenous Fbxw5 in $4 \mu \mathrm{g} \mathrm{HeLa}$ or 293T lysates (of not: unspecific band at $60 \mathrm{kDa}$, just below Fbxw5 signal; good seperation on $8 \%$ gels)

Immunoprecipitation: $\quad 5-10 \mu \mathrm{g} / \mathrm{mL}$ HeLa extract from $10^{8}$ cells

Immunofluorescence: no specific signal with formaldehyde fixation

\subsubsection{Mammalian cell lysate and extract preparation}

Whole cell lysates were prepared by lyzing cells in $1 \mathrm{x}$ or $2 \mathrm{x}$ SDS sample buffer after several washes with PBS at room temperature. Lysates were sonicated and boiled prior to SDS PAGE analysis. To load equal amounts of lysates, protein concentrations were determined using the Pierce $660 \mathrm{~nm}$ assay in combination 
with the ionic detergent compatibility reagent according to the manufacturer's instructions.

For extract preparation from commercially purchased HeLa S3 cell pellets (RELIATech), the pellet (approx. $15 \mathrm{~mL}$ wet volume) was thawed in two volumes of TB supplemented with $1 \mathrm{mM}$ DTT, aprotinin, leupeptin, pepstatin, PMSF. Cells were homogenized in a glass douncer using the $S$ pestle and the lysate was cleared by successive centrifugation steps at $4^{\circ} \mathrm{C}$ : $1500 \mathrm{xg}$ for $10 \mathrm{~min}, 25,000 \mathrm{x}$ $\mathrm{g}$ for $25 \mathrm{~min}$, and $100,000 \times \mathrm{g}$ for $60 \mathrm{~min}$. The supernatant from the last step (= HeLa cytosol) was aliquoted, flash frozen in liquid nitrogen, and stored at $-80^{\circ} \mathrm{C}$.

\subsubsection{Immunoprecipitation of endogenous proteins}

For immunoprecipitation (IP) of endogenous proteins (e.g. Fbxw5, Eps8, and Nek9), cell extracts were freshly prepared from HeLa suspension cells. For a typical IP, $10^{8}$ cells were collected by gentle centrifugation with $100 \mathrm{xg}$ and were washed two times with PBS containing $10 \mathrm{mM}$ NEM. The cell pellet was resuspended in $1 \mathrm{~mL}$ of cold TB buffer supplemented with $0.2 \%$ Tween20, 10 $\mathrm{mM}$ NEM, aprotinin, leupeptin, pepstatin, PMSF and was incubated on ice for 20 min (to allow for lysis of cells). The lysate was then cleared from cell debris by centrifugation with $100,000 \times \mathrm{g}$ at $4^{\circ} \mathrm{C}$ for $1 \mathrm{~h}$ and 5-10 $\mu \mathrm{g}$ antibody or control IgGs (from mouse, goat or rabbit) were added to the extract. After incubation under constant rotation at $4^{\circ} \mathrm{C}$ for $1 \mathrm{~h}, 7.5 \mu \mathrm{L}$ Protein $\mathrm{G}$ beads (Roche) for goat and mouse antibodies or $7.5 \mu \mathrm{L}$ Protein A beads (Roche) for rabbit antibodies were added. Samples were rotated at $4^{\circ} \mathrm{C}$ for another $2 \mathrm{~h}$ and beads collected by centrifugation in a swing-out rotor with $2000 \mathrm{xg}$ at $4^{\circ} \mathrm{C}$. Beads were washed three times with TB buffer supplemented with $0.2 \%$ Tween20, $10 \mathrm{mM} \mathrm{NEM}$, aprotinin, leupeptin, pepstatin, PMSF and were transferred to new tubes. Bound proteins were eluted with $2 x$ SDS sample buffer and were analyzed by SDS PAGE followed by immunoblotting.

For mass spectrometry analysis of endogenous Fbxw5 complexes, Fbxw5 was immunoprecipitated as described above; however, the IP was performed in the absence of NEM from HeLa cytosol prepared from commercially purchased HeLa cell pellets (RELIATech). 


\subsubsection{Mass spectrometry analysis}

Mass spectrometry analysis of flag-Fbxw5 and Fbxw5 immunoprecipitates

Mass spectrometry analysis of flag-Fbxw5 immunoprecipitates (obtained via transient transfection of five $15 \mathrm{~cm}$ dishes $293 \mathrm{~T}$ cells) was carried out in collaboration with Dr. Henning Urlaub (Max Planck Institute for Biophysical Chemistry, Göttingen). The gel lanes of both, the flag-Fbxw5 and control flag IP were cut into slices and each gel slice was subjected to in-gel digestion with trypsin (Roche). Peptides were extracted as described previously (Shevchenko et al. 1996) and were analyzed in a LTQ-Orbitrap mass spectrometer (Thermo Fisher Scientific) equipped with a nanoelectrospray ion source (ESI-LTQ Orbitrap MS). For analysis, obtained peptide sequences from each lane were merged and searched against the mammalian NCBInr protein database using the MASCOT search engine (www.matrixscience.com). Search parameters were: digestion with trypsin allowing for maximally 2 missed cleavages and carbamylation of cysteine residues and oxidation of methionine residues as variable modifications. Only protein hits with a score higher than 20 and absent in the control lane were considered as putative Fbxw5 interactors.

Mass spectrometry analysis of flag-Fbxw5 immunoprecipitates (obtained via $\alpha$ flag IP from five confluent 15c $\mathrm{m}$ dishes HEK 293T cells stably expressing flagFbxw5) and of Fbxw5 immunoprecipitates (from $2 \mathrm{~mL}$ of HeLa cytosol) was carried out by the core facility of mass spectrometry and proteomics of the $\mathrm{ZMBH}$, Heidelberg. Fbxw5 and control IP samples were separated by SDS PAGE (gradient 5-20\%) and stained with colloidal Commassie. A selection of band pairs of (flag-)Fbxw5 and control IPs were cut out and in-gel digested with trypsin. Peptides extracted from gel slices were analyzed in an ESI-LTQ Orbitrap mass spectrometer (Thermo Fischer Scientific) using standard conditions.

\section{Mapping of Nek9-dependent phosphorylation sites within Eps8}

To identify residues within Eps8 that are phosphorylated by Nek9 in vitro, CIPtreated mouse Eps8 and CIP- and Nek9-treated mouse Eps8 were analyzed viamass spectrometry by the lab of Henning Urlaub at the MPI-BPC in Götitngen. For this, protein samples were in-gel digested with chymotrypsin and peptides were extracted. Obtained peptides were analyzed in an ESI-LTQ Orbitrap mass 
spectrometer (Thermo Fischer Scientific) using standard conditions and identified peptide sequences were searched against the mammalian NCBInr protein database using the MASCOT search engine (www.matrixscience.com). Search parameters were: digestion with chymotrypsin allowing for maximally 2 missed cleavages and carbamylation of cysteine residues, oxidation of methionine residues, and phosphorlyation of serine, threonine, and tyrosine residues as variable modifications. Phosphopeptides were only found in the CIP- and Nek9treated Eps8 sample and therefore considered as Nek9-dependent.

\subsection{Cell biological techniques}

\subsubsection{Cultivation of adherent and suspension cells}

Adherent HeLa and HEK 293T cells were propagated in DMEM supplemented with $10 \%(\mathrm{v} / \mathrm{v}) \mathrm{FBS}$ at $37{ }^{\circ} \mathrm{C}$ and $5 \% \mathrm{CO}_{2}$. Usually, cells were split at a $1 / 10$ ratio just before reaching confluency. For this purpose, cells were washed with sterile PBS, detached from the culture dishes with trypsin/EDTA, and diluted with fresh medium.

HeLa suspension cells were propagated in Jokliks medium supplemented with 5 $\%(\mathrm{v} / \mathrm{v}) \mathrm{NCS}, 5 \%(\mathrm{v} / \mathrm{v})$ FBS. $2 \mathrm{mM}$ glutamine was added if the medium was older than two weeks. Cells were cultured in spinner flasks at $100 \mathrm{rpm}$ in a $37{ }^{\circ} \mathrm{C}$ incubator at $3-10 \times 10^{5} \mathrm{cells} / \mathrm{ml}$. The typical doubling time ranged between 16-24 h. The cell density was determined using a Neubauer counting chamber and cell density was adjusted daily.

To freeze cells, exponentially growing cells were trypsinized, diluted in serumcontaining medium, and collected by centrifugation with $100 \times \mathrm{g}$ for $5 \mathrm{~min}$. Cells were then resuspended in FBS supplemented with $10 \%$ DMSO $(\mathrm{v} / \mathrm{v})$, were aliquoted, and were slowly frozen enclosed in a 2-propanol insulation at $-80^{\circ} \mathrm{C}$. For long-term storage, cells were transferred to liquid nitrogen tanks. 


\subsubsection{Transient transfection of mammalian cells}

Transfection of mammalian cells with plasmids

HEK 293T cells were transfected with plasmids using the calcium phosphate precipitation method. Per $10 \mathrm{~cm}$ dish of cells, 20-28 /g DNA were supplemented with 86.8 / $\mathrm{L} 2.5 \mathrm{M}$ calcium chloride and sterile water to a final volume of 700 ( $\mathrm{L}$. This mixture was subsequently mixed with equal parts of 2-fold concentrated HBS buffer pH 7.05 (50 mM HEPES, $270 \mathrm{mM}$ sodium chloride, $1 \mathrm{mM} \mathrm{KCl}, 1.5 \mathrm{mM}$ disodium hydrogen phosphate, $0.2 \%$ glucose, $\mathrm{pH}$ titrated with $0.5 \mathrm{M}$ sodium hydroxide, sterile-filtered) by adding the HBS drop-wise onto the DNA/calcium chloride solution under constant vortexing (in order to generate small calcium phosphate/DNA precipitates). The mixture was incubated for 10-20 minutes at room temperature and was then added to the cells. Generally the medium was changed $6-12 \mathrm{~h}$ after transfection. Cells were typically harvested $24-48 \mathrm{~h}$ post transfection. For $15 \mathrm{~cm}$ dishes, the amount of transfection mix was doubled.

HeLa cells were transfected by FUGENE6.0, Lipofectamine ${ }^{2000}$ (both cationic lipidbased transfection reagents), or JetPRIME ${ }^{\mathrm{TM}}$ (a non-liposomal transfection reagent) according to the manufacturer's instructions. Typical DNA / transfection reagent ratios per well of a 6 well plate were: 2 /g DNA / 6 /L FuGENE6.0; 2 /g DNA / 2-3 /L Lipofectamine ${ }^{2000}, 2$ /g DNA / 4 /L JetPRIME ${ }^{\text {TM }}$.

\section{Transfection of mammalian cells with siRNAs}

To downregulate different genes (e.g. Fbxw5, Cul1, or Cul4A/B) in mammalian cells, siRNA-mediated gene silencing was employed (Elbashir et al. 2001). For this, HeLa cells were transfected using Lipofectamine RNAiMax, according to the manufacturer's instructions. In short, HeLa cells in 6-well plates (20-30\% confluent) were transfected with 10-20 nM siRNA and 6 (L Lipofectamine RNAiMAX reagent per well. $4 \mathrm{~h}$ post transfection, the medium was changed. $48 \mathrm{~h}$ post transfection, cells were typically split 1:1, 1:2, and 1:4 and analyzed by immunoblotting $72 \mathrm{~h}, 96 \mathrm{~h}$, and, $120 \mathrm{~h}$ post transfection, respectively. 


\subsubsection{Generation and selection of stable flag-Fbxw5 HEK 293T cells}

HEK 293T cells were transfected with pCDNA3.1-flag-Fbxw5 by the calcium phosphate precipitation method as first described by (Graham and van der Eb 1973). 20 (g DNA per $10 \mathrm{~cm}$ dish were used. Cells were split $24 \mathrm{~h}$ after transfection, selection with $1 \mathrm{fg} / \mathrm{ml} \mathrm{G} 418$ was started 2 days after transfection. The efficiency of selection was controlled on untransfected HEK 293T cells. Transfected cells were cultured under selection by renewing the selection medium every 3-4 days. After approximately 3 weeks, single cells expanded to visible cell colonies. Single colonies were scraped off the dish with a sterile pipet tip, transferred into a drop of trypsin to separate the cells, and single clones were expanded to a 10 or $15 \mathrm{~cm}$ format. Different colonies were tested for flag-Fbxw5 expression by western blot analysis of SDS cell lysates. Selection with G418 was maintained until the clones were frozen for long-term storage. This way, three stable cell lines were created: one with low (clone \# 2), one with medium (clone \# 4) and one with high (clone \#5) flag-Fbxw5 expression levels. For all immunoprecipitations presented in this work, clone \# 5 was used.

\subsubsection{Manipulation of degradation pathways in mammalian cells}

\section{Proteasome inhibitor treatment of HeLa cells}

To inhibit proteasome activity, HeLa cells (60-80 \% confluent) were incubated with 20 (M MG132 for 2-6 h. In control conditions, HeLa cells were treated with appropriate concentrations of DMSO (since MG132 was dissolved in DMSO).

\section{Induction and blockage of autophagy}

To induce autophagy, HeLa cells $(60-80 \%$ confluent) were washed two times with PBS and were then incubated with DMEM without FBS for 2-14 $\mathrm{h}$ (serum starvation). To block lysosome-dependent degradation via repression of endosomal acidification (Mizushima et al. 2008), untreated HeLa cells or Hela cells serum starved for $12 \mathrm{~h}-14 \mathrm{~h}$ were incubated with $20 \mathrm{mM}$ ammonium chloride for $2 \mathrm{~h}-6 \mathrm{~h}$. 


\section{RESULTS}

\section{Fbxw5 assembles into SCF and CRL4 complexes}

Originally, Fbxw5 was classified into the F-box family of proteins due to the presence of an N-terminal F-box domain and its association with Skp1 in pull down assays (Winston et al. 1999). Since then, the functions and properties of Fbxw5 have remained largely elusive and have become even more enigmatic with the finding that it also is a DDB1 and Cul4-associated factor (DCAF, (He et al. 2006); raising the intriguing possibility that Fbxw5 acts as a substrate recognition subunit in context of two distinct Cullin-RING based E3 ligases (CRLs). To investigate this hypothesis and to clarify, in which complexes Fbxw5 assembles in cells, I started my PhD work with the immunoblot analysis of Fbxw5 immunoprecipitates.

\subsection{Fbxw5 predominantly assembles into SCF complexes}

To obtain large and pure amounts of Fbxw5-containing E3 ligase complexes from cells via immunoprecipitation (IP) in a reproducible manner, I decided to generate HEK 293T cell lines stably expressing flag-Fbxw5. For this, HEK 293T cells were transfected with pCDNA3.1-flag-Fbxw5 and after selection with the antibiotic G418, single cell clones were selected and tested for flag-Fbxw5 expression. In this approach, I was able to establish three different stable HEK 293T cell lines (data not shown). One of these cell lines (clone \# 5) was then used for purification of flag-Fbxw5 by $\alpha$-flag immunoprecipitation followed by peptide elution. For a single experiment, five $15 \mathrm{~cm}$ plates of nearly confluent cells were used. Western blot analysis of elution fractions revealed that Fbxw5 interacts with both, components of the SCF complex (Skp1, Cul1, and Rbx1) and components of the Cul4A-DDB1 complex (Figure 9A). Furthermore, comparing input with immunoprecipitated fractions, we observed that Fbxw5 interacts with a larger cellular fraction of Cul1 than Cul4A. Taking into account that both cullin homologues are expressed abundantly in HEK 293T cells with only a slight excess of Cul4A over Cul1 (unpublished observation of the Harper lab), we 
concluded that Fbxw5 is predominantly assembled into SCF complexes under normal cellular growth conditions.

A)

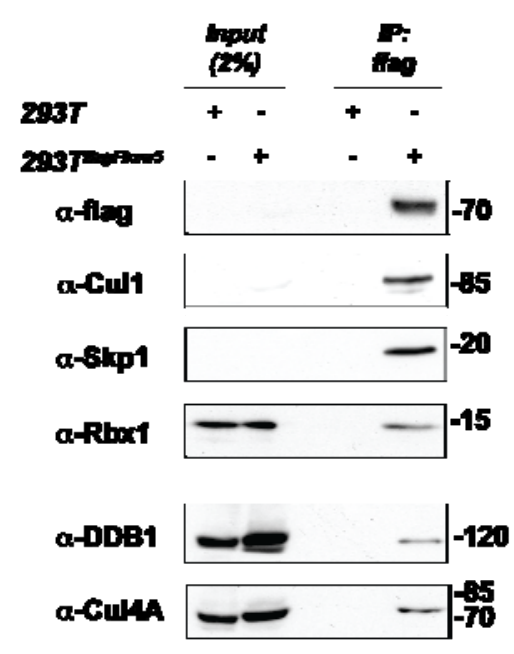

c)

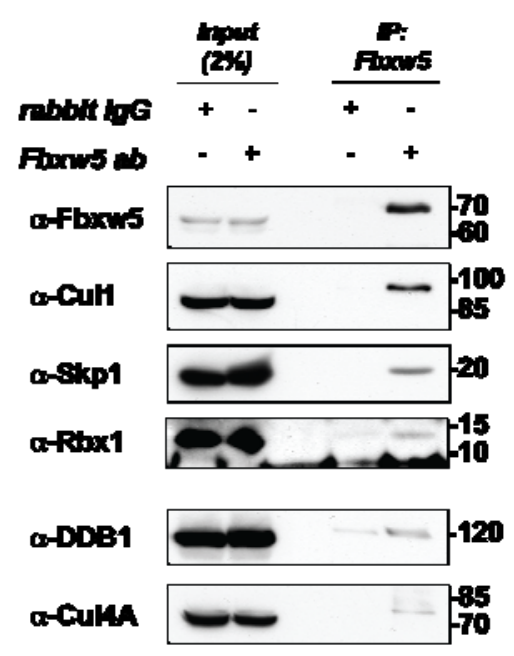

B)

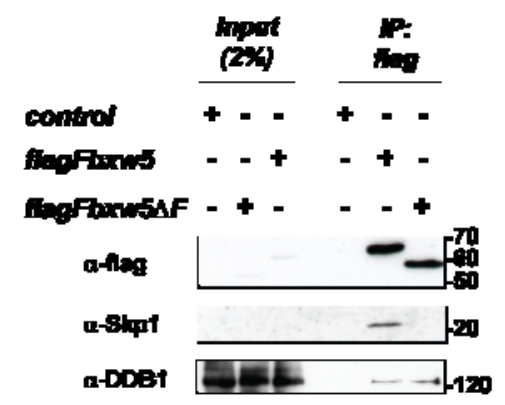

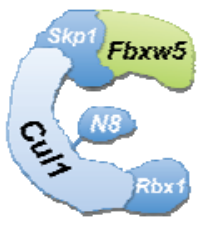

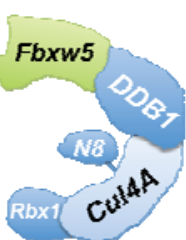

Faxw5 5AF-box

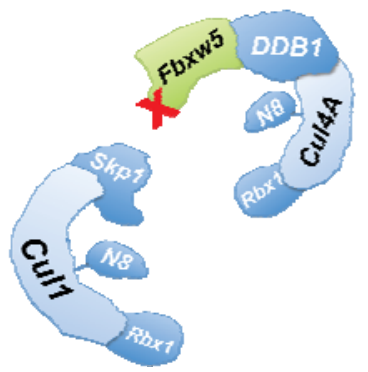

Figure 9: Fbxw5 interacts with components of both Cul1- and Cul4-based E3 ligases. A) Five $15 \mathrm{~cm}$ dishes of confluent HEK 293T cells and HEK 293T cells stably expressing flag-Fbxw5 were lyzed under native conditions in two pellet volumes of flag-Fbxw5 buffer (see Materials and Methods 2.2.5, page 63). Lysates were subjected to flag-IP using 20 $\mu$ L ANTI-FLAG-M2 agarose followed by flag peptide elution. B) $10^{8} \mathrm{HeLa}$ CSH suspension cells were lyzed under native conditions in two pellet volumes of lysis buffer (see Material and Methods 2.2.11, page 68). Lysates were subjected to Fbxw5-IP using $5 \mu \mathrm{g}$ of affinity-purified Fbxw5 antibodies (see Material and Methods 2.2.9, page 66) followed by elution in SDS sample buffer. C) Experiment was performed as described in A) but using five $15 \mathrm{~cm}$ dishes of HEK 293T cells transiently transfected with pCDNA3.1-Flag (control), pCDNA3.1-Flag-Fbxw5, or pCDNA3.1-Flag-Fbxw5 $\Delta \mathrm{F}-$ box.

To verify the specificity of these immunoprecipitation experiments, we next compared co-purifying proteins of flag-Fbxw5 from HEK 293T cells to those of a flag-Fbxw5 version lacking the $\mathrm{N}$-terminal F-box domain (flag-Fbxw5 $\Delta \mathrm{F}$-box). As 
shown in Figure 9B, roughly equal amounts of flag-Fbxw5 and flag-Fbxw $5 \Delta \mathrm{F}-\mathrm{box}$ were obtained via flag-IP. While full-length Fbxw5 interacted with both, Skp1 and DDB1, the F-box deletion mutant only interacted with DDB1. These findings demonstrated the specificity of the interactions and confirmed previous findings that Fbxw5 interacted with the linker protein of SCF ligases, Skp1, via its F-box domain (Hu et al. 2008). For interaction with the linker of CRL4 ligases, DDB1, a more C-terminal region of the protein (most likely two DWD boxes) is required (He et al., 2006).

To investigate whether Fbxw5 would also interact with components of these two types of $C R L$ complexes in an endogenous scenario and to exclude overexpression artifacts, polyclonal antibodies against Fbxw5 were raised in rabbits (for characterization see Material and Methods 2.2.9, page 66) and employed to immunoprecipitate Fbxw5 and interacting proteins from HeLa suspension cells. As depicted in Figure 9C, the results of the flag-Fbxw5 IP could be confirmed: endogenous Fbxw5 preferentially assembled into SCF complexes.

A)

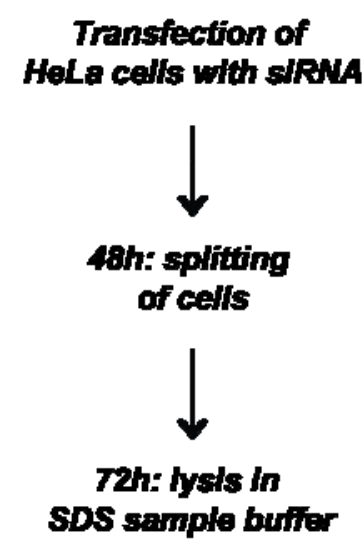

B)

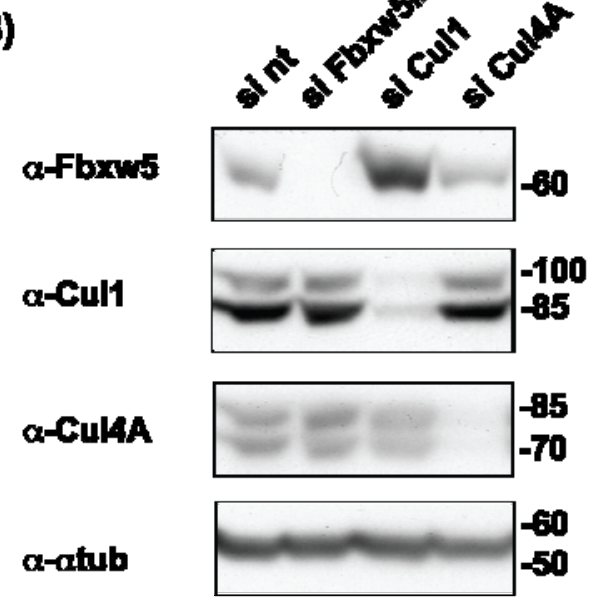

Figure 10: Downregulation of Cul1 stabilizes Fbxw5 levels in HeLa cells. A) Experimental flow chart of the siRNA experiment depicted in B B) Immunoblot analysis of HeLa lysates from cells treated with $10 \mathrm{nM}$ non-targeting (nt) siRNA or siRNAs against Fbxw5, Cul1, Cul4A, or Cul4B for $72 \mathrm{~h}$.

One mechanism how F-box proteins are believed to be degraded in cells is Ubiquitin-dependent degradation in an autocatalytic mechanism within the SCF complex (Galan and Peter 1999). Therefore, we reasoned that if Fbxw5 is indeed 
preferentially contained within SCF complexes, alterations of Cul1 levels in cells should also influence Fbxw5 stability. Downregulation of Cul1 in HeLa cells by siRNA treatment confirmed our hypothesis (Figure 10): in Cul1-silenced cells, Fbxw5 levels were significantly stabilized, while they remained unchanged upon downregulation of Cul4A.

Taken together, these results indicate that Fbxw5 is principally capable of forming two distinct $\mathrm{CRL}$ complexes. However, the predominant complex under normal cellular growth conditions seems to be the SCF complex. 


\section{Fbxw5 interacts with Eps8 and Nek9}

Up to date, only one target for Fbxw5 has been described. Hu and coworkers provided biochemical and biological evidence that Fbxw5 - in context of a Cul4ADDB1 complex - is involved in the degradation of the tuberous sclerosis complex protein 2 (TSC2) (Hu et al. 2008). Since our own data suggested that Fbxw5 predominantly assembles into SCF complexes, we were next interested in identifying novel interaction partners and targets for Fbxw5. For this, we performed mass spectrometry analysis of Fbxw5 immunoprecipitates from different sources.

2.1. Mass spectrometry and western blot analysis of Fbxw5 immunoprecipitates reveal novel Fbxw5 interactors

In a first approach to identify novel Fbxw5-interacting proteins, flag-Fbxw5 was transiently expressed in HEK 293T cells and purified via $\alpha$-flag immunoprecipitation (Figure 11A). As control, an $\alpha$-flag IP from the same amount of mock-transfected cells was performed in parallel. Flag-Fbxw5 and control peptide eluates were separated via SDS Page, were Coomassie-stained (Figure 11B), and were sent for mass spectrometry analysis to the lab of Henning Urlaub at the MPI-BPC, Göttingen. Entire gel lanes were cut into several sections; single gel slices were digested with trypsin and analyzed by ESI-MS. MS data originating from slices of the same lane were merged and searched against the mammalian database using MASCOT. Comparing the two data sets, I could identify 22 proteins that were only present in the flag-Fbxw5 and not in the control IP (Table 1). Amongst them were Fbxw5 itself and already known Fbxw5 interaction partners such as Cul1, DDB1, and two Cul1-interacting proteins (subunit 1 and 4 of the COP9 signalosome), clearly demonstrating that the approach itself was suitable for finding direct or indirect Fbxw5 interaction partners and emphasizing that all other proteins within the list were putative novel Fbxw5 interactors. 
A)

\section{translont transfaction: flag-Fbxw5 or empty vector}

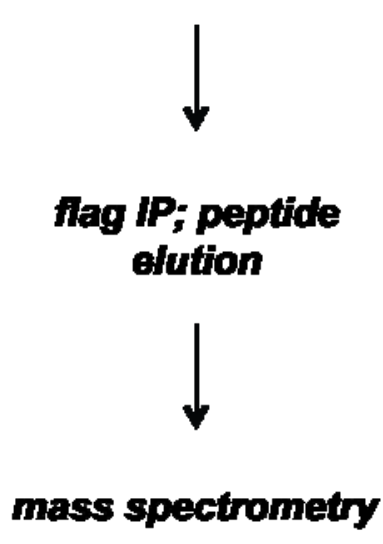

B)

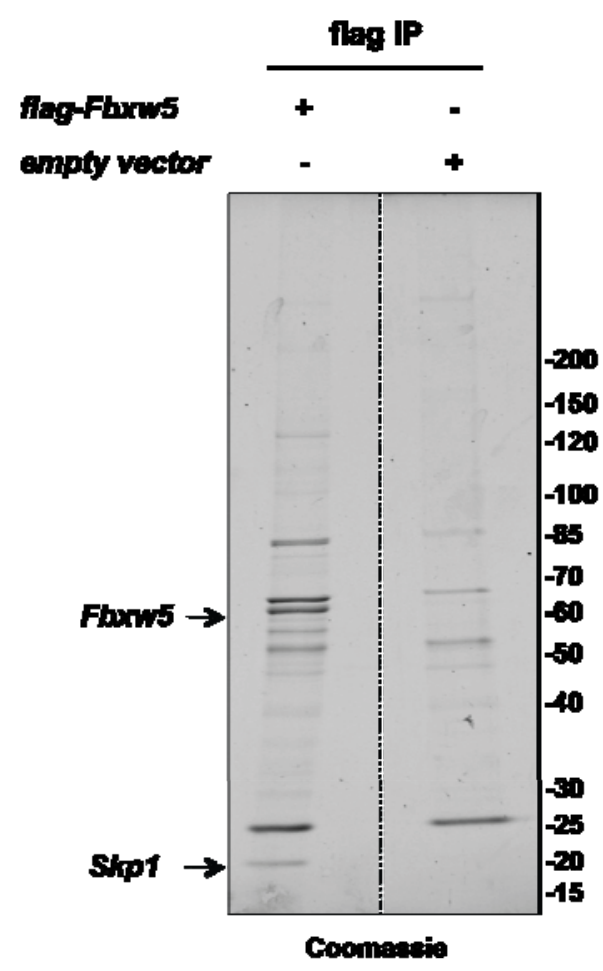

Figure 11: Mass Spectrometry analysis of flag-Fbxw5 immunoprecipitates. A) Experimental flow chart: flag-Fbxw5 was purified via $\alpha$-flag immunoprecipitation from five $15 \mathrm{~cm}$ dishes of transiently transfected HEK 293T cells followed by flag peptide elution. As control, the IP was also performed from HEK 293T cells transfected with empty pCDNA3.1 vector. Flag-Fbxw5 and control immunoprecipitates were separated via SDS Page and Coomassie stained (see B). Entire gel lanes were cut into several sections; single gel slices were digested with trypsin and analyzed by ESI-MS by the lab of Henning Urlaub at the MPI-BPC in Göttingen. B) Coomassie gel of the mass spectrometry experiment.

Table 1: Proteins specifically identified upon transient transfection and IP of flag-Fbxw5. Listed are proteins that were only present in the flag-Fbxw5 and not in the control IP with a protein score higher than 20. In addition, the accession number, molecular weight (in Da), and the number of peptides identified for each protein are shown. Already known interaction partners of Fbxw5 are highlighted in blue; novel interaction partners that could be validated by Western blotting are highlighted in red.

\begin{tabular}{|c|c|c|c|c|}
\hline Protein & Accession & MW & Score & $\begin{array}{c}\text { peptide } \\
\text { matches }\end{array}$ \\
\hline $\begin{array}{c}\text { F-box and WD repeat domain } \\
\text { containing 5 (Fbxw5) [Homo } \\
\text { sapiens] }\end{array}$ & gi|24308129 & 63882 & 760 & 70 \\
\hline \begin{tabular}{c} 
Cullin1 (CUL1) [Homo sapiens] \\
\hline $\begin{array}{c}\text { pyrroline 5-carboxylate synthetase } \\
\text { [Homo sapiens] }\end{array}$
\end{tabular} & gi|1381142 & 87333 & 510 & 16 \\
\hline
\end{tabular}




\begin{tabular}{|c|c|c|c|c|}
\hline $\begin{array}{c}\text { centaurin beta2 / ACAP2 [Homo } \\
\text { sapiens] }\end{array}$ & gi|4688902 & 87848 & 237 & 8 \\
\hline elF-4 gamma [Homo sapiens] & gi|219613 & 153342 & 138 & 5 \\
\hline $\begin{array}{l}\text { valosin-containing protein [Homo } \\
\text { sapiens] }\end{array}$ & gi|6005942 & 89266 & 117 & 4 \\
\hline $\begin{array}{l}\text { NIMA-related kinase Nek9 [Homo } \\
\text { sapiens] }\end{array}$ & gi|18997185 & 107081 & 96 & 3 \\
\hline $\begin{array}{c}\text { Epidermal growth factor receptor } \\
\text { pathway substrate } 8 \text { (Eps8) [Homo } \\
\text { sapiens] }\end{array}$ & gi|20988309 & 91852 & 90 & 3 \\
\hline $\begin{array}{l}\text { COP9 complex subunit } 1 \text { (SGN1) } \\
\text { [Homo sapiens] }\end{array}$ & gi|2494624 & 53394 & 78 & 4 \\
\hline $\begin{array}{c}\text { Probable ATP-dependent RNA } \\
\text { helicase DDX17 (DEAD box protein } \\
\text { 17) [Homo sapiens] }\end{array}$ & gi|3122595 & 72326 & 74 & 1 \\
\hline $\begin{array}{l}\text { COP9 complex subunit } 4 \text { (SGN2) } \\
\text { [Homo sapiens] }\end{array}$ & gi|5410300 & 46169 & 72 & 3 \\
\hline $\begin{array}{c}\text { hypothetical protein LOC144097 } \\
\text { [Homo sapiens] }\end{array}$ & gi|39930523 & 41011 & 70 & 2 \\
\hline $\begin{array}{c}\text { fatty acid binding protein } 5 \text { (psoriasis- } \\
\text { associated) [Homo sapiens] }\end{array}$ & gi|4557581 & 15155 & 53 & 2 \\
\hline $\begin{array}{l}\text { ZincFinger Type } \mathrm{CCHH} \text { antiviral } \\
\text { protein [Homo sapiens] }\end{array}$ & gi|16550682 & 67520 & 52 & 1 \\
\hline $\begin{array}{l}\text { myristoylated alanine-rich C-kinase } \\
\text { substrate (MARCKS) [Homo sapiens] }\end{array}$ & gi|187385 & 32750 & 38 & 1 \\
\hline $\begin{array}{c}\text { phosphoribosyl pyrophosphate } \\
\text { synthetase-associated protein } 2 \\
\text { [Homo sapiens] }\end{array}$ & gi|4506133 & 40899 & 30 & 1 \\
\hline $\begin{array}{l}\text { small nuclear ribonucleoprotein } \\
\text { 70kDa polypeptide[Homo sapiens] }\end{array}$ & gi|36100 & 70040 & 30 & 1 \\
\hline hCG18527 [Homo sapiens] & gi|119581610 & 13756 & 26 & 2 \\
\hline $\begin{array}{c}\text { FAST kinase domains } 5 \text { [Homo } \\
\text { sapiens] }\end{array}$ & gi|11141903 & 86519 & 23 & 1 \\
\hline $\begin{array}{l}\text { DNA damage-binding protein } 1 \\
\text { (DDB1) [Homo sapiens] }\end{array}$ & gi|418316 & 126901 & 22 & 1 \\
\hline $\begin{array}{c}\text { zinc finger/RING finger } 2 \text { [Homo } \\
\text { sapiens] }\end{array}$ & gi|23821044 & 24100 & 22 & 1 \\
\hline Ubiquitin [Homo sapiens] & gi|229532 & 8446 & 22 & 1 \\
\hline
\end{tabular}


For verification of candidates, antibodies against a selection of the identified proteins were commercially purchased and used for western blot analysis of flagFbxw5 immunoprecipitates. As depicted in Figure 12, flag-Fbxw5 immunoprecipitates specifically contained the epidermal growth factor receptor pathway substrate 8 (Eps8) and the NIMA-related kinase 9 (Nek9), demonstrating that Eps8 and Nek9 indeed co-purified with overexpressed Fbxw5. In contrast, the Arf-GAP with coiled-coil, ANK repeat, and PH domain-containing protein 2 (ACAP2) was detected in the flag-Fbxw5 bound as well as in the control fraction, suggesting unspecific binding of ACAP2 to antibodies or beads during the IP and indicating the presence of false positive hits within the list of putative Fbxw5 interactors.

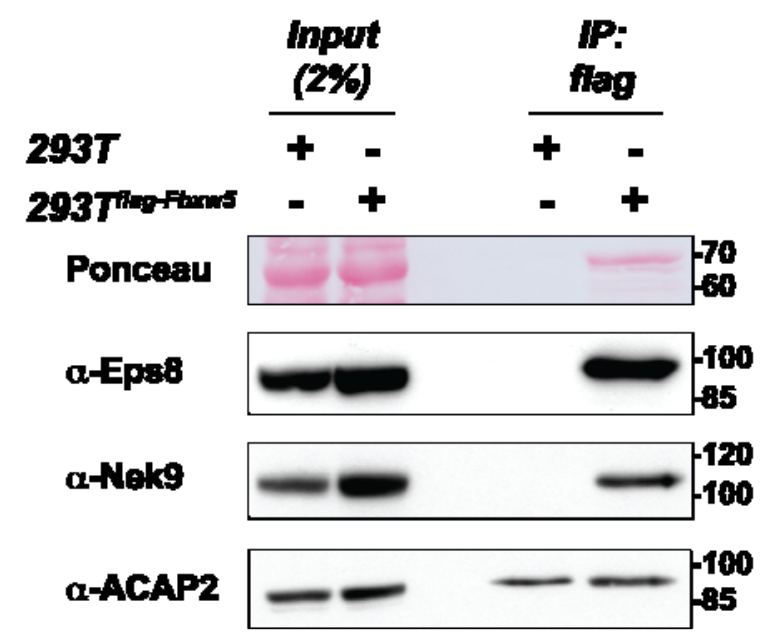

Figure 12: Eps8 and Nek9 but not ACAP2 specifically interact with flag-Fbxw5. flagFbxw5 was immunoprecipitated from five $15 \mathrm{~cm}$ dishes of stable HEK 293T cells followed by peptide elution and immunoblot analysis against target candidates from the first mass spectrometry screen. $\alpha$-flag IP from the same amount of HEK 293 T cells served as control. For western blot analysis, $50 \%$ of the total peptide eluates were loaded.

To minimize these false positives and to further verify and expand the list of putative Fbxw5-interacting proteins in a reliable manner, we decided to include two additional mass spectrometry screens in our search for Fbxw5 interactors. Therefore, flag-Fbxw5 IP fractions from stable HEK 293T cell lines and endogenous Fbxw5 IP fractions from HeLa S3 cells were analyzed by mass spectrometry; this time together with the core facility for proteomics of the ZMBH in Heidelberg. Both mass spectrometry experiments were performed in a similar manner as the first one with the following modifications: not entire lanes were analyzed, but single bands only visible in the Fbxw5 IP fraction were cut out in both, control and Fbxw5 IP lanes and subjected to ESI-MS analysis. Data analysis for each experiment was performed separately by only selecting for 
proteins that could be detected specifically in the Fbxw5-bound fraction. Then, lists of identified proteins of these two screens were manually compared to the list shown in Table 1. Only proteins that had been identified by two out of the three mass spectrometry screens were considered to be interaction partners of Fbxw5 with high confidence. Applying these criteria, I obtained - next to CRL components and the already verified interactors Eps8 and Nek9 - four more Fbxw5 interaction candidates: the probable ATP-dependent RNA helicase DEAD box protein 17 (DDX17), the actin cytoskeleton remodeler myristoylated alaninerich C-kinase substrate (MARCKS), the transcription intermediary factor 1-beta Tif1 $\beta /$ TRIM28, and the nucleolar protein nucleolin (Table 2). Out of these, TRIM28 could be verified as interaction partner of flag-Fbxw5 by western blot: in immunoprecipitation experiments (in which Eps8, Nek9, and the already published Fbxw5 target TSC2 were present in the flag-Fbxw5 bound fraction) also TRIM28 co-purified with Fbxw5 in a specific manner (Figure 13). DDX17, MARCKS, and nucleolin have not been tested so far.

In summary, mass spectrometry analysis of Fbxw5 immunoprecipitates from different sources allowed the identification of six novel interaction partners of Fbxw5 (Table 2): three of these (Eps8, Nek9, and TRIM28) co-purified with flagFbxw5 by western blot; the remaining three (MARCKS, Ddx17, and Nucleolin) need to be tested. Furthermore, the published Fbxw5 target TSC2 could be verified as an interactor by immunoblotting.

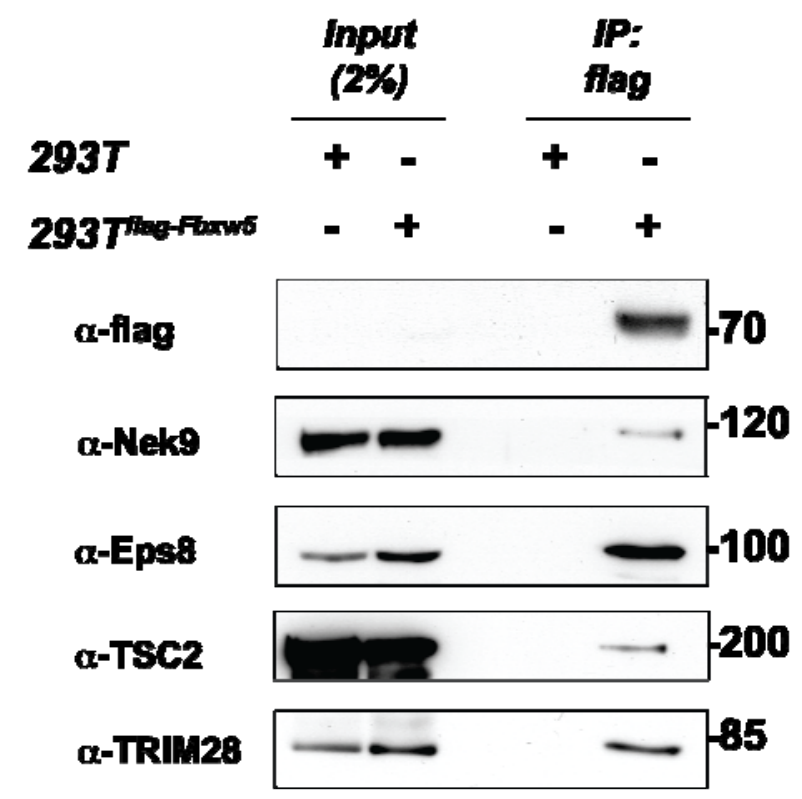

Figure 13: flag-Fbxw5 interacts with Nek9, Eps8, TRIM28 and TSC2. flagFbxw5 was immunoprecipitated from five $15 \mathrm{~cm}$ dishes of stable HEK 293T cells followed by peptide elution and immunoblot analysis against different target candidates. $\alpha$-flag immunoprecipitations from the same amount of HEK 293T cells served as control. For western blot analysis, $50 \%$ of the total peptide eluates were loaded. 
Table 2: Summary of novel Fbxw5 interaction partners identified by mass spectrometry or western blot analysis. Listed proteins were either identified in two different mass spectrometry screens as specific interaction partners or could be verified as Fbxw5 interactors by western blot analysis of flag-Fbxw5 immunoprecipitates. (+) Detected, $(-)$ not detected, $\left({ }^{*}\right)$ not detected, but analyzed gel slices might have been unsuitable for detection of the indicated protein since only single gel slices were analyzed. Mass spectrometry analysis of flag-Fbxw5 immunoprecipitates from transiently transfected cells was carried out by the lab of Henning Urlaub, MPI-BPC in Göttingen and mass spectrometry analysis of the other two screens was performed by the core facility of proteomics at the ZMBH in Heidelberg.

\begin{tabular}{|c|c|c|c|c|}
\hline & \multicolumn{3}{|c|}{ MASS SPECTROMETRY } & WESTERN \\
\hline $\begin{array}{c}\text { Protein } \\
\text { detected } \\
\text { in ... }\end{array}$ & $\begin{array}{c}\text {... flag-Fbxw5 } \\
\text { IP upon } \\
\text { transient } \\
\text { transfection }\end{array}$ & $\begin{array}{c}\text {... flag-Fbxw5 } \\
\text { IP upon stable } \\
\text { transfection }\end{array}$ & $\begin{array}{c}\text {... Fbxw5 IP } \\
\text { (endogenous } \\
\text { from HeLa) }\end{array}$ & $\begin{array}{l}\text {... flag- } \\
\text { Fbxw5 IP }\end{array}$ \\
\hline \multicolumn{5}{|c|}{ Published interaction partners } \\
\hline TSC2 & - & * & * & + \\
\hline \multicolumn{5}{|c|}{ Novel interaction partners } \\
\hline Eps8 & + & * & * & + \\
\hline Nek9 & + & + & * & + \\
\hline$D d \times 17$ & + & + & + & to be tested \\
\hline MARCKS & + & + & * & to be tested \\
\hline TRIM28 & - & + & + & + \\
\hline Nucleolin & - & + & + & to be tested \\
\hline
\end{tabular}

\subsection{Fbxw5 endogenously interacts with Eps8 and Nek9}

Up to this point, the Fbxw5 interaction partner validation had been carried out by immunoblot analysis of flag-Fbxw5 immunoprecipitates. To rule out that the observed interactions had been caused by an overexpression artifact, I decided 
to immunoprecipitate endogenous Fbxw5 from HeLa suspension cells and test for co-purification of Eps8 and Nek9. For this, $10 \mu \mathrm{g}$ of antibodies were employed to IP Fbxw5 from $1 \mathrm{~mL}$ HeLa cytosol and $50 \%$ of the Fbxw5-bound fraction was analyzed by SDS PAGE followed by western blotting (Figure 14). Compared to the total cellular protein pool, a small percentage of both, Eps8 and Nek9 copurified with Fbxw5; neither protein was detectable in the IP with rabbit IgGs. These observations demonstrated that Fbxw5 directly or indirectly binds to Eps8 and Nek9 also in an endogenous scenario.

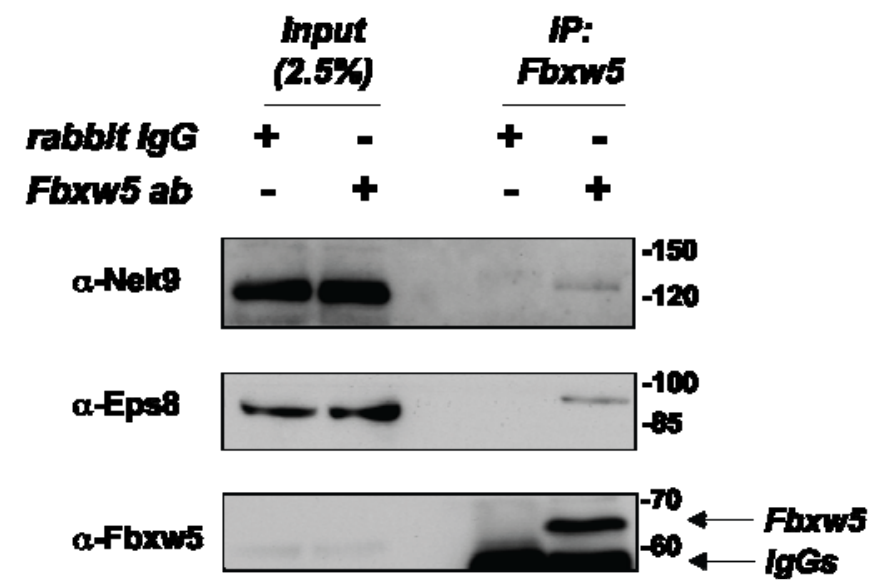

Figure 14: Fbxw5 endogenously interacts with Eps8 and Nek9. $10 \mu \mathrm{g}$ Fbxw5 antibodies or rabbit IgGs were each incubated with $1 \mathrm{~mL}$ HeLa lysate and subsequently precipitated using Protein $A$ beads. Half of the antibodybound fractions were loaded on a gel and analyzed by $\alpha$-Fbxw5, $\alpha$-Eps8, and $\alpha$-Nek9 immunoblotting.

\subsection{Fbxw5 interacts with Nek9 and Eps8 independently of its F-box domain}

To further investigate the interaction of Fbxw5 with Nek9 and Eps8, I re-probed samples of the flag-Fbxw5 and flag-Fbxw5 $\Delta \mathrm{F}-$ box immunoprecipitation experiments depicted in Figure $9 \mathrm{C}$ by $\alpha$-Eps 8 and $\alpha$-Nek9 immunoblotting (Figure 15). Compared to the IP from mock-transfected cells, Eps8 and Nek9 specifically co-purified with both, flag-Fbxw 5 and flag-Fbxw $5 \Delta \mathrm{F}$-box. These findings indicated that the interaction between Fbxw5, Nek9 and Eps8 does not require the F-box domain and hence ruled out the possibility that Eps8 and Nek9 binding to Fbxw5 is bridged by Skp1 or Cul1. 


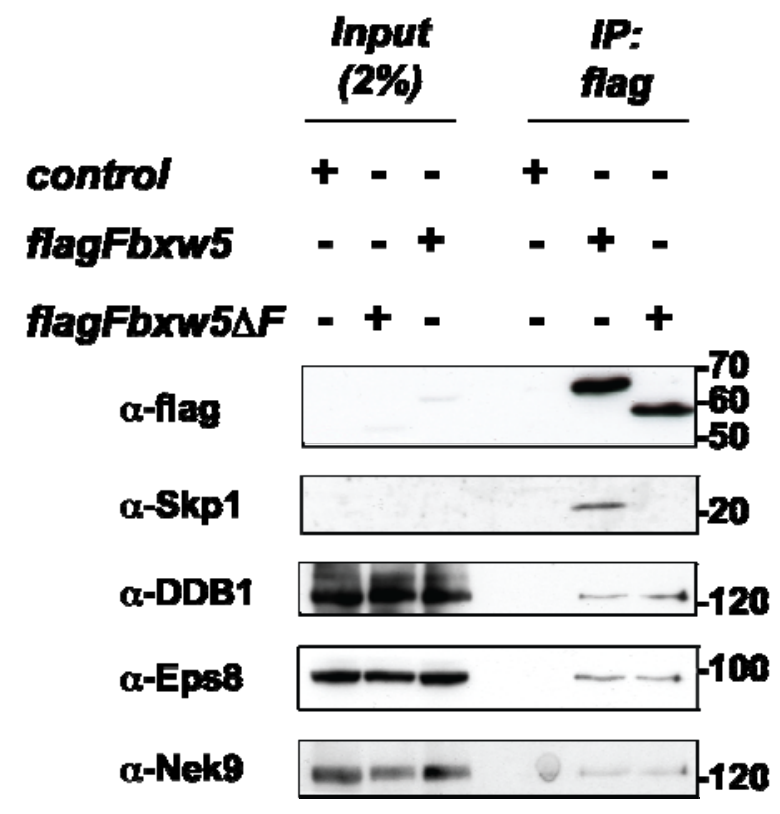

Figure 15: The interaction between Fbxw5, Nek9 and Eps8 does not require Fbxw5's F-box domain. Samples of the flag-Fbxw5 and flag-Fbxw5 $\Delta$ Fbox immuno-precipitation experiment depicted in Figure 9C were reprobed with anti-Eps8 and anti-Nek9 antibodies. $30 \%$ of the antibody-bound fractions were loaded on the gel.

Considering that Fbxw5 endogenously recognized Eps8 and Nek9 and that these interactions were not mediated by other SCF complex components, we decided to study the biological meaning of these interactions. 


\section{Eps8 is a substrate of $\mathrm{SCF}^{\mathrm{Fbxw} 5}$}

The epidermal growth factor pathway substrate 8 (Eps8) is a bifunctional actin remodeler (Hertzog et al. 2010), which has been shown to participate in the formation of several functionally distinct macromolecular complexes. This way, Eps8 can act as a key player in integrating signals from receptor tyrosine kinase (RTK)-activated pathways leading to actin cytoskeleton remodeling via Rac and to receptor endocytosis via Rab5 (reviewed in (Di Fiore and Scita 2002)). Furthermore, Eps8 has been shown to promote cell proliferation (Fazioli et al. 1993), constitutive phosphorylation of Eps8 in tumor cell lines contributes to malignant transformation (Matoskova et al. 1995), and increasing levels of Eps8 in oral squamous carcinoma cells correlate with enhanced cell migration and invasion (Yap et al. 2009). These studies indicate that tight and proper regulation of Eps8 levels and activity are crucial for normal cellular growth and motility. Nevertheless, astonishingly little is known about how the protein levels of Eps8 are controlled and how its recruitment into functionally distinct complexes is regulated within cells.

Having demonstrated that Fbxw5 endogenously recognizes Eps8 (Figure 14), we found it intriguing to speculate that Eps8 could be a substrate for Fbxw5 and that Fbxw5-mediated ubiquitylation of Eps8 could influence its levels or functions. To test this hypothesis, cell biological approaches in combination with in vitro ubiquitylation assays were employed.

\subsection{Fbxw5 regulates Eps8 levels in vivo}

The most common way of how SCF and other cullin-RING based Ubiquitin E3 ligase (CRL) complexes regulate their substrates is by promoting Ubiquitindependent degradation (Petroski and Deshaies 2005). Hence, to obtain some first evidence that Eps8 is indeed a target of Fbxw5, we first tested whether alterations of Fbxw5 levels within cells would influence Eps8 stability. 


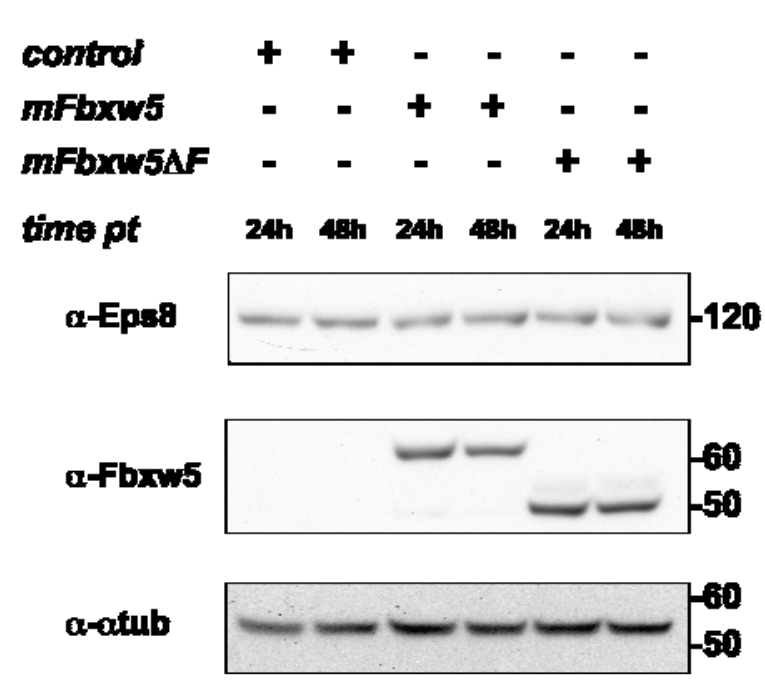

Figure 16: Overexpression of Fbxw5 or Fbxw5 $\Delta$ F-box does not influence steady state levels of Eps8 in HeLa cells. HeLa cells were transfected with pIRES-hrGFP1a (control), pIRES-hrGFP1a-mFbxw5, or pIRES hrGFP1a-mFbxw5 $\Delta$ F-box using JetPRIME $^{\text {TM }}$ reagent. 24 and $48 \mathrm{~h}$ post transfection, cells were harvested by direct lysis in 2xSDS sample buffer and analyzed by SDS PAGE followed by immunoblotting. An $\alpha$ - $\alpha$-tubulin immunoblot ( $\alpha$-tub) served as loading control.

Overexpression of Fbxw5 in HeLa cells has no influence on Eps8 levels

To test, whether overexpression of Fbxw5 would have an influence on Eps8 levels, I transiently transfected HeLa cells with untagged mFbxw5 or mFbxw $5 \Delta \mathrm{F}$ box in context of a pIRES-hrGFP vector. From $16 \mathrm{~h}$ post transfection on, approximately $70-80 \%$ of the cells were transfected as judged by GFP expression. Cells were lyzed directly in 2x SDS sample buffer 24 and $48 \mathrm{~h}$ post transfection and the Eps8 and Fbxw5 levels were investigated by western blotting (Figure 16). Endogenous Fbxw5 could not be seen in western blot exposures that allowed detection of $\mathrm{mFbxw} 5$ and $\mathrm{mFbxw} 5 \Delta \mathrm{F}$-box in the linear range, clearly indicating that these proteins were significantly overexpressed (lanes 3-6, Figure 16). Despite this fact, Eps8 levels were not changed in these conditions 24 or 48 $\mathrm{h}$ post transfection when compared to the cells transfected with empty control vector. Consistent with this, further experiments to detect cellular ubiquitylated Eps8 species with or without Fbxw5 overexpression were also unsuccessful and proteasome inhibitor treatment of HeLa cells suggested that Eps8 is a stable protein under normal growth conditions (data not shown). One explanation for these observations could be a tight regulation of the ubiquitylation and degradation of Eps8 cells. While overexpressed Fbxw5 is integrated into functional E3 ligase complexes (as demonstrated by ubiquitylation assays, see below Figure 18), Eps8 in cells might be sequestered in complexes and thus not recognizable by Fbxw5. Only upon a certain stimulus it might be released and 
ubiquitylated by Fbxw5. To further complicate matters, Eps8 ubiquitylation might be readily reversed by specific isopeptidases, rendering its detection difficult.

To circumvent at least some of these putative problems, we next took the reverse approach: downregulation of Fbxw5 in cells.

\section{Downregulation of Fbxw5 results in Eps8 accumulation in HeLa cells}

To silence Fbxw5 in cells, siRNA-mediated knock down was employed. For this, I designed three different siRNAs targeting Fbxw5 (siFbxw5 \#1-3) using an online tool from Ambion (siRNA target finder) and also ordered one pre-designed siRNA (siFbxw5 \#4). 20-30\% confluent HeLa cells were transfected with $10 \mathrm{nM}$ of each siRNA for $48 \mathrm{~h}$, split, and incubated for another $48 \mathrm{~h}$. After direct lysis in 2x SDS sample buffer, levels of Fbxw5 and Eps8 were analyzed by immunoblotting (Figure 17A and B). All four employed siRNAs mediated efficient knock down of Fbxw5, and resulted in a medium (siFbxw5 \#1, \#2, and \#4) or strong (siFbxw5 \#3) increase of Eps8 levels, indicating that Fbxw5 indeed promotes degradation of Eps8 in vivo. Unfortunately, it was impossible to convincingly correlate the extent of Eps8 increase with residual Fbxw5 levels, since remaining Fbxw5 levels upon siRNA knock down were too little for quantative analysis. In addition, the effect of elevated Eps8 levels upon Fbxw5 knock down could only be observed when precisely following the schedule of the siRNA experiment (Figure 17A). A likely reason for this observation is that the culturing conditions within these experimental settings induced signals required for Fbxw5-mediated Eps8 degradation, while this was not the case for other approaches. Experiments dedicated to identify these signals (e.g. investigation of an involvement of EGF receptor or integrin signaling) did not give any conclusive results (data not shown).

To collect further evidence that the observed effect was specific for Fbxw5, I performed rescue experiments. HeLa cells were transfected with $10 \mathrm{nM}$ nontargeting siRNA or siFbxw5 $\# 3$ for $48 \mathrm{~h}$, split, and $24 \mathrm{~h}$ later transfected with flagmFbxw5 that is resistant against the siRNA or a control plasmid. After another 24 $\mathrm{h}$ of incubation, cell lysates were analyzed by western blot (Figure 17C). As expected, Fbxw5-silenced cells showed elevated Eps8 levels when compared to cells treated with non-targeting siRNA (lanes 1 - 2, Figure 17C), also after 
transfection with a control plasmid. However, in the presence of exogenous flagmFbxw5, this Eps8 elevation was reduced (lane 2 versus lane 4, Figure 17C). In light of the fact that only $40-50 \%$ of the cells were transfected with the rescue plasmid (as judged by GFP control transfections that were performed in parallel), this is a significant effect.

A)

Transfection of Hela cells with siRNAs

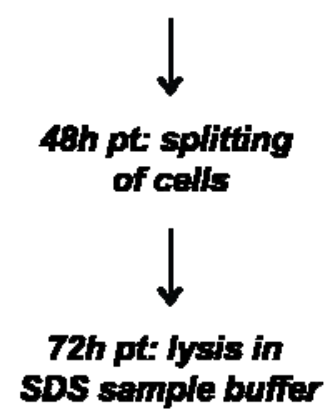

B)

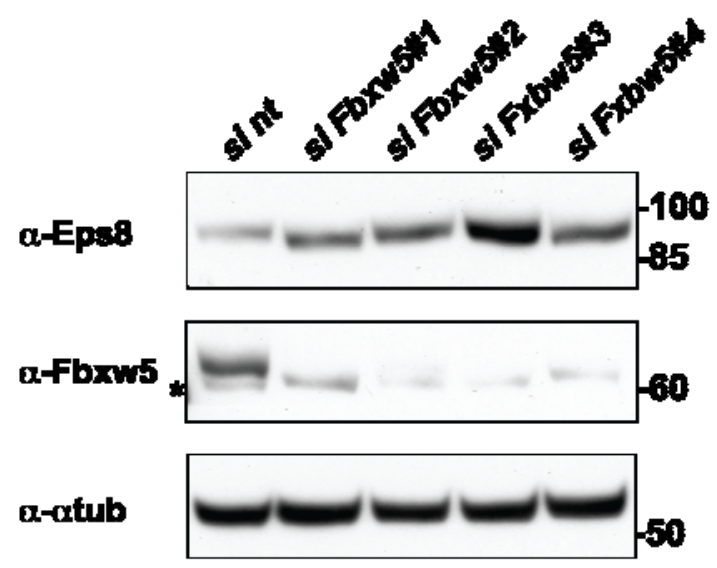

c) control flag-mFbxw5

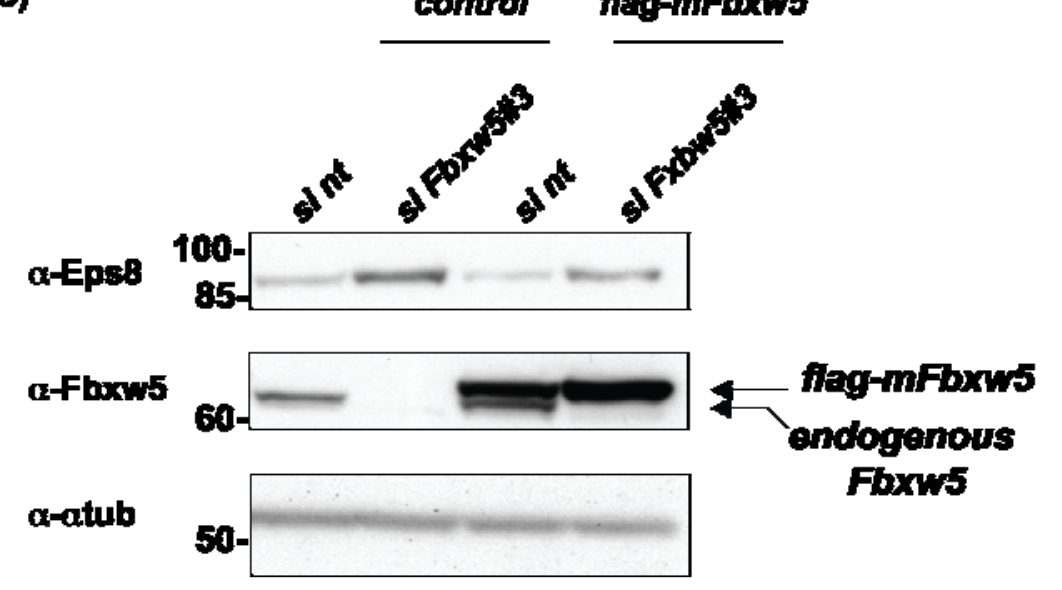

Figure 17: Fbxw5 knock down leads to an increase in Eps8 levels. A) Experimental flow chart. HeLa cells were transfected with $10 \mathrm{nM}$ of non-targeting siRNA (si nt) or four different siRNAs targeting Fbxw5 (siFbxw5 \#1-4) using Lipofectamine RNAiMAX. $48 \mathrm{~h}$ post transfection, cells were split once and harvested by direct lysis in $2 x$ SDS sample buffer $72 \mathrm{~h}$ post siRNA transfection. B) $\alpha$-Eps8, $\alpha$-Fbxw5 and $\alpha-\alpha$-tubullin immunoblot analysis of the experiment described in A). C) Fbxw5 rescue experiment. HeLa cells were transfected with $10 \mathrm{nM}$ of nontargeting siRNA (si nt) or siFbxw5 \#3. $48 \mathrm{~h}$ post transfection cells were split and were transfected with pCDNA3.1-flag-mFbxw5 or pCDNA3.1 as control $24 \mathrm{~h}$ later. $96 \mathrm{~h}$ post siRNA transfection cells were harvested by direct lysis in $2 x$ SDS sample buffer followed by immunoblot analysis using the indicated antibodies. $\alpha-\alpha$-tubulin immunoblots ( $\alpha$-tub) served as loading control. ${ }^{*}=$ unspecific band 
In summary, downregulation of Fbxw5 with four different siRNAs resulted in an increase of Eps8 levels that could be rescued by overexpression of exogenous murine Fbxw5, indicating that Fbxw5 promotes Eps8 degradation in vivo. While the signaling events leading to the Eps8 elevation upon Fbxw5 downregulation could not be completely elucidated and need to be further investigated in the future, these findings strongly suggested that Eps8 is a substrate for Fbxw5dependent ubiquitylation.

\section{2. $\mathrm{SCF}^{\mathrm{Fbxw5}}$ ubiquitylates Eps8 in vitro}

Flag-Fbxw5 but not flag-Fbxw5 4 Fbox immunoprecipitates stimulate in vitro ubiquitylation of Eps8

For testing whether Fbxw5 indeed exhibits Ubiquitin E3 ligase activity towards Eps8, I decided to set up an in vitro ubiquitylation assay. For this, the basic ubiquitylation machinery including Ubiquitin, the Ubiquitin E1 enzyme Ube1, and the Ubiquitin E2 enzyme UbcH5b were recombinantly purified according to standard protocols (Material and Methods, section 2.2.3, page 56) and incubated together with His-Eps8 (from SF9 cells, provided by Giorgio Scita) and ATP. As source of E3 ligase activity, flag-IP fractions from HEK 293T cells or HEK 293T cells stably expressing flag-Fbxw5 were titrated into the reactions (Figure 18A). $\alpha$ Eps8 immunoblotting indicated that in the absence of ATP (lanes 1-3, Figure 18A) and in absence of any IP fraction (lane 4, Figure 18A) Eps8 was not modified with Ubiquitin. However, if increasing amounts of flag-Fbxw5 were added into the reaction (lanes 5-6, Figure 18A), higher migrating bands of Eps8 up to the top of the gel appeared. These ubiquitylated species of Eps8 were generated in dependence of Fbxw5, since such a dramatic increase in Eps8 ubiquitylation could not be observed in presence of control IP fractions (lanes 7-8, Figure 18A). Therefore, we concluded that flag-Fbxw5 immunoprecipitates indeed contain Ubiquitin E3 ligase activity towards Eps8. 


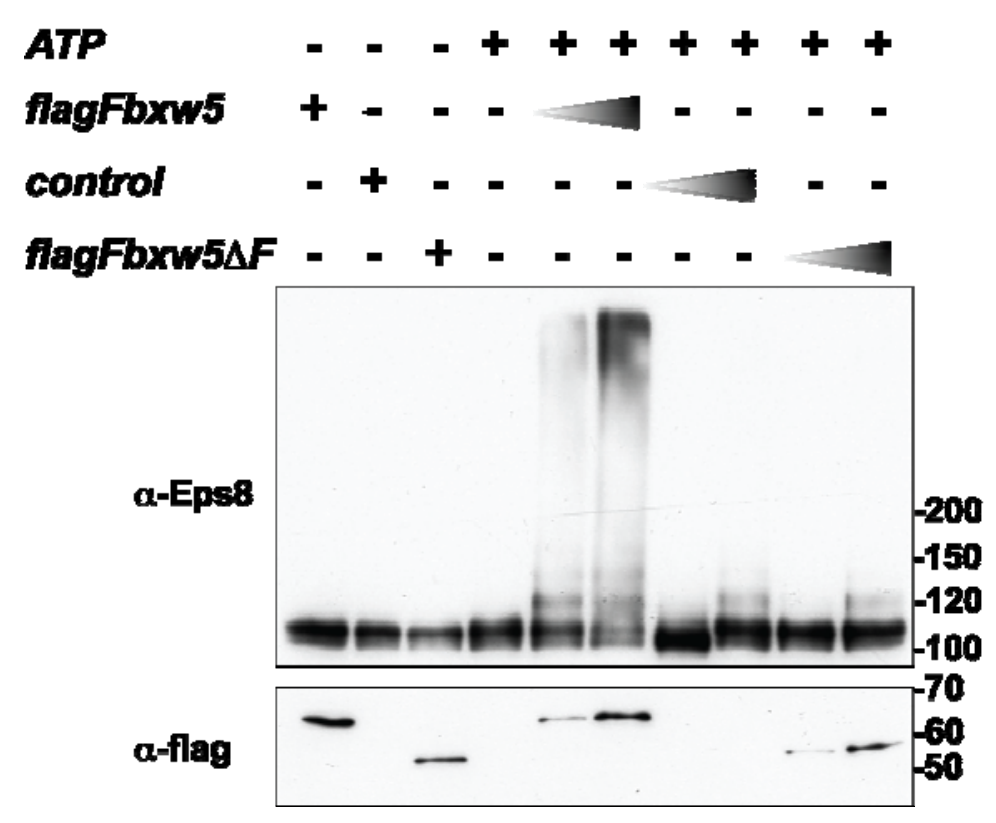

Figure 18: Eps8 is ubiquitylated by flag-Fbxw5 but not by flag-Fbxw5 $\Delta \mathrm{F}-$ box immunoprecipitates in vitro. A) $0.1 \mu \mathrm{M}$ His-Eps8 (from SF9 cells) were incubated together with $75 \mu \mathrm{M}$ His-Ubiquitin, $170 \mathrm{nM}$ Ube1, $1 \mu \mathrm{M}$ UbcH5b, $5 \mathrm{mM}$ ATP in the absence or presence of different amounts of flag-Fbxw5 and flag-Fbxw5 4 Fbox immunoprecipitates (corresponding to approximately 3 and $6 \%$ of an IP fraction from five $15 \mathrm{~cm}$ dishes transiently transfected HEK $293 \mathrm{~T}$ cells) at $30^{\circ} \mathrm{C}$ for $120 \mathrm{~min}$.

Since flag-Fbxw5 immunoprecipitates contained components of SCF and CRL4 ligases (Figure 9A), we next addressed the question, which Fbxw5-containing complex exhibited the ubiquitylation activity towards Eps8 in the in vitro assay. For this, flag-Fbxw5 $\Delta \mathrm{F}$-box immunoprecipitates, which only contained components of CRL4 complexes (Figure 9C), were used in ubiquitylation assay of Eps8. With amounts of flag-Fbxw5 $\Delta \mathrm{F}$-box that were comparable to those of the full-length protein ( $\alpha$-flag immunoblot Figure $18 \mathrm{~A}$, lanes $5+6$ versus $9+10$ ), no significant Eps8 ubiquitylation was observed. Importantly, this loss of ubiquitylation activity was not caused by simple reduction of target binding due to the deletion in Fbxw5, since Eps8 co-purified with flag-Fbxw5 and flag-Fbxw5 $\Delta \mathrm{F}-$ box in equal amounts (Figure 15). Hence, we could conclude, that the Fbxw5dependent Ubiquitin E3 ligase activity towards Eps8 within the in vitro assays was strictly dependent on the presence of SCF complexes. To exclude that DDB1 and Cul4A or other unknown factors, which might co-purify with Fbxw5 during flag-IP, contributed to Eps8 ubiquitylation, we next decided to in vitro reconstitute $\mathrm{SCF}^{\mathrm{Fbxw5}}$ complexes. 
In vitro reconstituted $\mathrm{SCF}^{\mathrm{Fbxw5}}$ ubiquitylates Eps8

To directly test whether $\mathrm{SCF}^{\mathrm{Fbxw5}}$ is an $\mathrm{E} 3$ ligase for Eps8 in ubiquitylation assays, we decided to in vitro reconstitute $\mathrm{SCF}^{\mathrm{Fbxw5}}$ in a similar manner as previously described for $\mathrm{SCF}^{\mathrm{Skp} 2}$ (Li et al. 2005). In this approach, the entire SCF complex is assembled from individually purified Nedd8 Cul1-Rbx1 and Skp2-Skp1 subcomplexes. Therefore, in order to be able to apply this protocol to $\mathrm{SCF}^{\mathrm{Fbxw} 5}$ reconstitution (Figure 19), I had to establish a protocol for Fbxw5-Skp1 purification. After several futile attempts to obtain soluble human Fbxw5 or Fbxw5-Skp1 complex from bacteria (for details see Material and Methods section 2.2.3, page 56), human Fbxw5-Skp1 complex was successfully purified from SF9 cells (Material and Methods section 2.2.4, page 61). In a collaborative effort with Matthew Calabrese from Brenda Schulman's lab, I was also able to produce soluble mouse Fbxw5-Skp1 complex in bacteria (Material and Methods, section 2.2.3, page 56). These Fbxw5-Skp1 complexes were then used for $\mathrm{SCF}^{\mathrm{Fbxw} 5}$ reconstitution by mixing them with equimolar amounts of Nedd8 Cul1-Rbx1 complex (provided by Brenda Schulman) followed by incubation on ice for $20 \mathrm{~min}$.

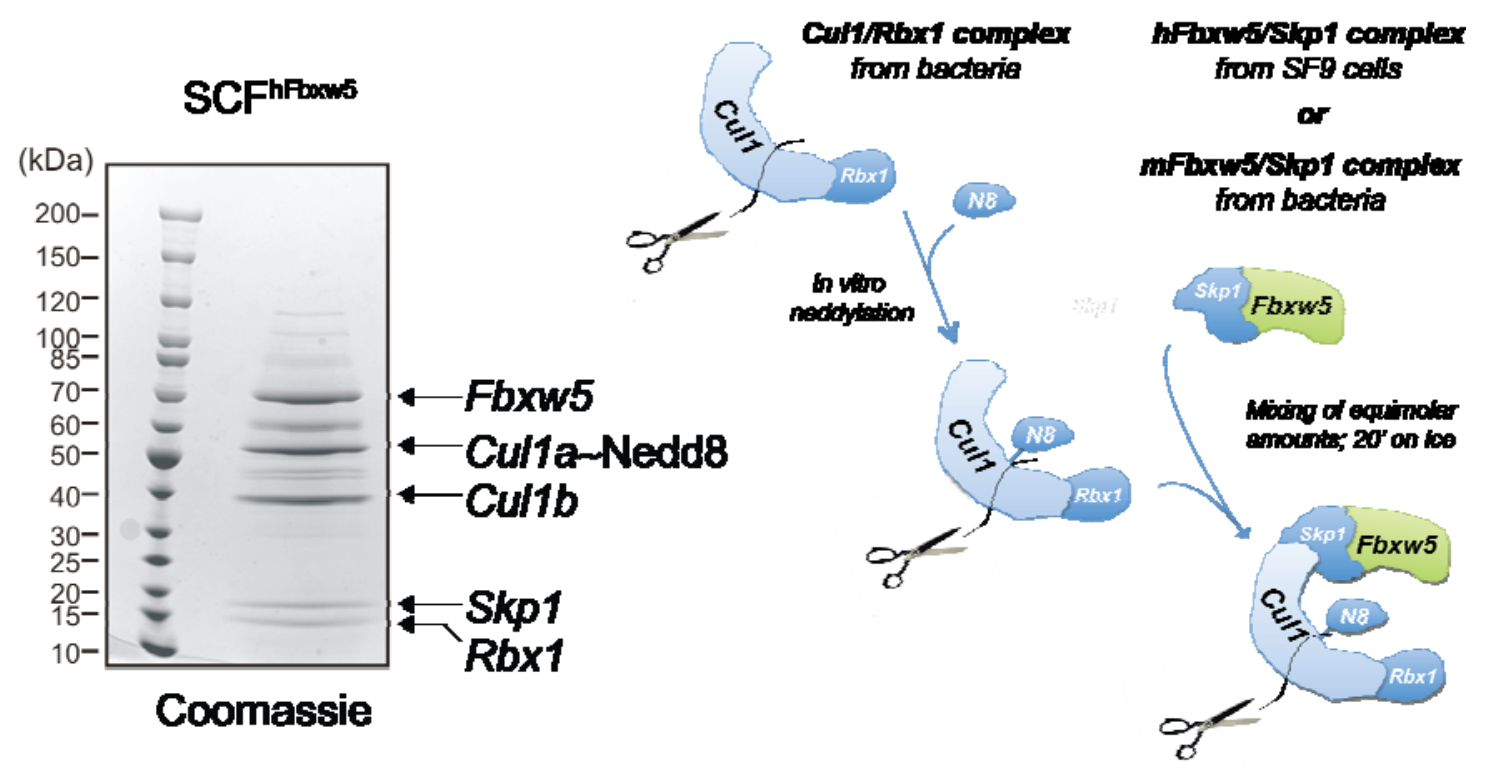

Figure 19: $\mathrm{SCF}^{\mathrm{Fbxw5}}$ can be reconstituted in vitro. For $\mathrm{SCF}^{\mathrm{Fbxw5}}$ reconstitution, human Fbxw5/Skp1 complex was purified from SF9 cells via $\mathrm{Ni}^{2+}$ pull down followed by gel filtration or, alternatively, mouse Fbxw5/Skp1 complex was purified from E.coli. The Cul1-Rbx1 complex was purfied from E.coli using a "split and co-express" approach and in vitro neddylated as previously described (Li et al. 2005) (Duda et al. 2008). The recombinant Nedd8 Cul1-Rbx1 complex used throughout this study was provided by Brenda Schulman. For complex formation, both subcomplexes were mixed in equimolar amounts and incubated on ice for $20 \mathrm{~min}$. Applying this procedure, $\mathrm{SCF}^{\mathrm{Fbxw5}}$ complexes of reasonable purity can be obtained as shown by the Coomassie-stained gel on the right. 
Recombinant $\mathrm{SCF}^{\mathrm{Fbxw5}}$ complexes generated this way were next used in ubiquitylation assays of Eps8 (Figure 20). Recombinant His-Eps8 (provided by Giorgio Scita) was incubated together with His-Ubiquitin, Ube1, Cdc34 or UbcH5b, and ATP in the presence or absence of in vitro reconstituted $\mathrm{SCF}^{\mathrm{Fbxw} 5}$. As depicted in Figure 20A, Eps8 is only efficiently modified if the whole ubiquitylation machinery including $\mathrm{SCF}^{\mathrm{Fbxw} 5}$ is present in the reaction (lanes 3 and 5 , Figure 20A), clearly indicating that the reaction is $\mathrm{SCF}^{\mathrm{Fbxw}}$-dependent. This is true for either $\mathrm{UbcH} 5 \mathrm{c}$ or $\mathrm{Cdc} 34$, the E2 enzymes known to work together with SCF complexes (Saha and Deshaies 2008). Importantly, under the same experimental conditions, a control protein, RanGAP1, was not significantly modified (lanes 7 and 9, Figure 20A).

A)

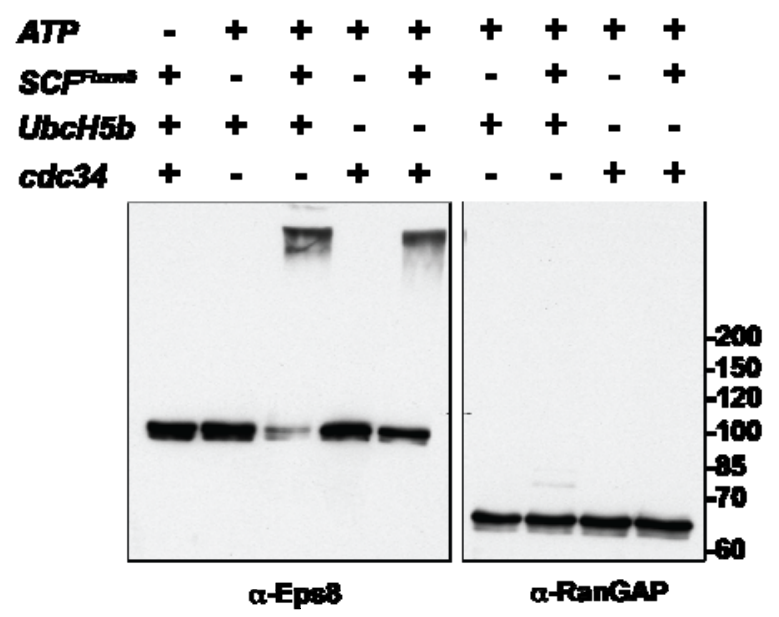

B)

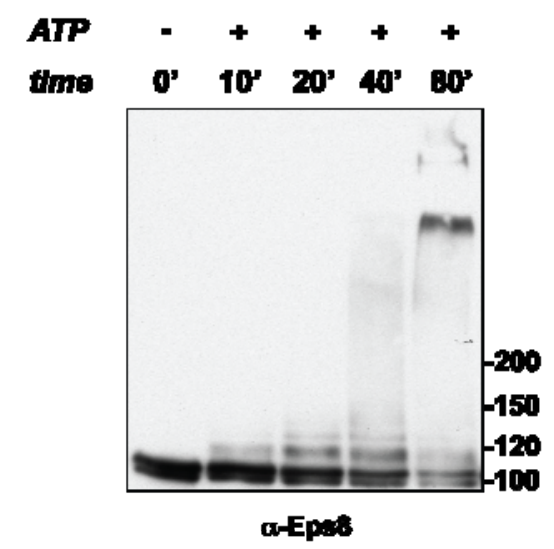

Figure 20: $\mathrm{SCF}^{\mathrm{Fbxw5}}$ ubiquitylates Eps8 in vitro. A) $0.1 \mu \mathrm{M}$ His-Eps8 or RanGAP1 were incubated together with $75 \mu \mathrm{M}$ His-Ubiquitin, $170 \mathrm{nM}$ Ube1, $1 \mu \mathrm{M}$ UbcH5b/Cdc34, $5 \mathrm{mM}$ ATP in the absence and presence of $150 \mathrm{nM} \mathrm{SCF}^{\mathrm{hFbxw} 5}$ at $30^{\circ} \mathrm{C}$ for $90 \mathrm{~min}$. B) Time course of SCF ${ }^{\mathrm{hFbxw}}$ dependent Eps8 ubiquitylation. $0.1 \mu \mathrm{M}$ His-Eps8 were incubated together with $75 \mu \mathrm{M}$ HisUbiquitin, $170 \mathrm{nM}$ Ube1, $1 \mu \mathrm{M} \mathrm{UbcH5b}, 150 \mathrm{nM} \mathrm{SCF}^{\mathrm{Fbxw} 5}$, and $5 \mathrm{mM}$ ATP at $30^{\circ} \mathrm{C}$ and the reaction was stopped at different time points. Detection was carried out by imunoblotting with the indicated antibodies.

Taken together, these findings demonstrated that Eps8 is a target of $\mathrm{SCF}^{\mathrm{Fbxw5}}$ dependent ubiquitylation. To our knowledge, this is the first target to be described for Fbxw5 in context of the SCF complex. 
3.3. Fbxw5-mediated ubiquitylation of Eps8 does not seem to require phosphorylation

Target recognition by F-box proteins usually requires phosphorylation of the substrate (Skaar et al. 2009). Since Eps8 is a known phopsphoprotein (Olsen et al. 2006; Rikova et al. 2007; Gauci et al. 2009; Menna et al. 2009) and we purified Eps8 for ubiquitylation assays from mammalian and insect cells, it was conceivable that Eps8 carried phosphorylations that could influence its $\mathrm{SCF}^{\mathrm{Fbxw5}}$ dependent ubiquitylation.

\subsubsection{Eps8 from insect cells is quantitatively phosphorylated}

One way of testing whether Eps8 phosphorylation is required for its $\mathrm{SCF}^{\mathrm{Fbxw5}-}$ mediated ubiquitylation would be to use Eps8 purified from bacteria in in vitro assays. However, from unpublished data of the labs of Giorgio Scita and Per Paolo Di Fiore, we knew that it is challenging to obtain properly folded and functional full length Eps8 from bacteria. Hence, we took a different approach by removing phosphorylations of Eps8 purified from SF9 cells via treatment with Calf intestine phosphatase (CIP). As depicted in Figure 21, incubation of Eps8 with CIP resulted in an increase in electrophoretic mobility of the complete pool of Eps8 compared to a mock-treated Eps8 fraction. This finding indicated that Eps8 is quantitatively phosphorylated when purified from SF9 cells and that these modifications can be efficiently reversed by CIP treatment in vitro.

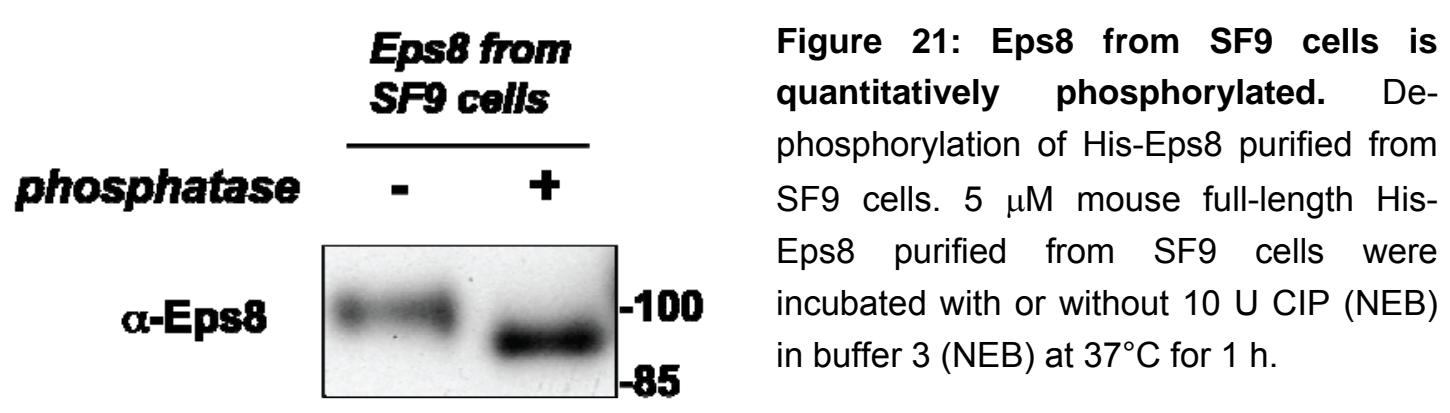

3.3.2. Phosphatase treatment of Eps8 has no major impact on its Fbxw5mediated ubiquitylation

To test whether Eps8 requires phosphorylations for $\mathrm{SCF}^{\mathrm{Fbxw5}}$-mediated ubiquitylation, Eps8 was purified from SF9 cells, subjected to a treatment with or 
without CIP, and both pools of Eps8 were compared for ubiquitylation in time course experiments (Figure 22). In the absence of ATP in the ubiquitylation reactions, Eps8 treated with phosphatase migrated faster compared to the nontreated Eps8, indicating that phosphorylations were efficiently removed (lane 1 and 4, Figure 22B). Interestingly, $\mathrm{SCF}^{\mathrm{Fbxw5}}$ did not significantly discriminate between these different pools of Eps8, as no major difference in ubiquitylation efficiency could be observed after 30 and 60 min (lanes 2 and 3 vs. lanes 5 and 6 , Figure 22B). Nevertheless, a minor decrease in ubiquitylation efficiency for CIP-treated Eps8 cannot be completely ruled out, since in some experiments the remaining unmodified Eps8 seemed to be a bit more prominent in the phosphatase-treated samples compared to the mock-treated Eps8 fractions. Yet, this observation could be misleading, since phosphatase-treated Eps8 runs in sharper bands on SDS PAGE compared to the phosphorlyated forms.

A)

\section{Purfication of Eps8 from SF9 cells}

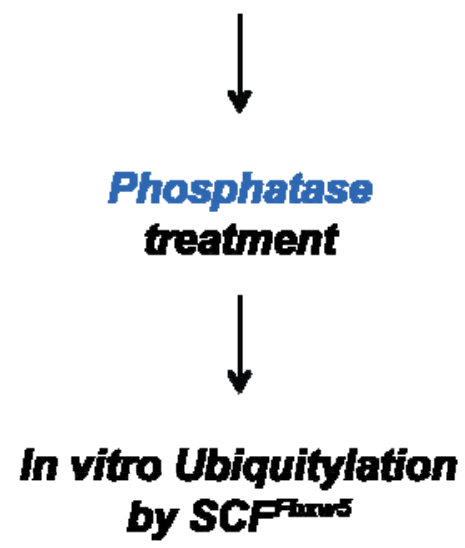

B)

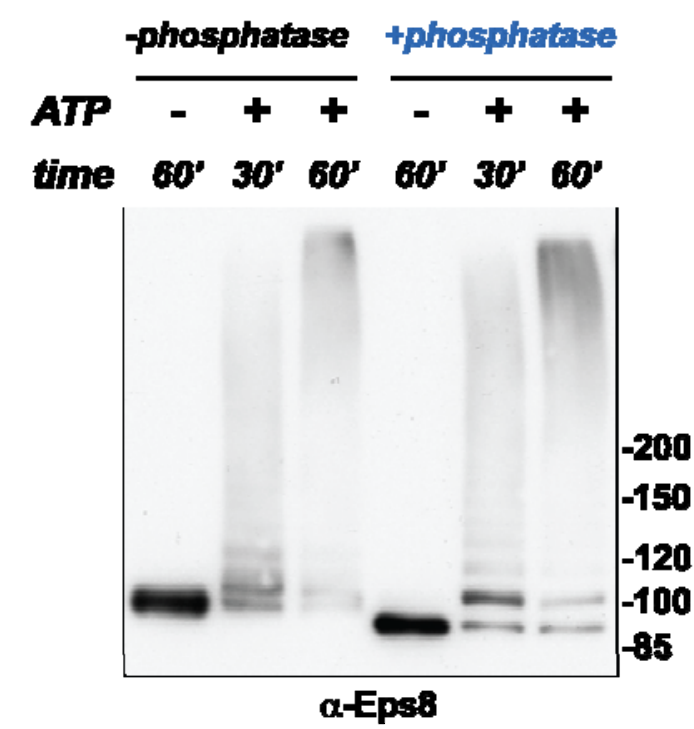

Figure 22: Phosphatase treatment of Eps8 has no major impact on its ubiquitylation by SCF $^{\text {Fbxw5 }}$. A) Experimental flow chart. $5 \mu \mathrm{M}$ mouse full length His-Eps8 purified from SF9 cells were subjected to dephosphorylation by incubation with (+ phosphatase) or without (- phosphatase) $10 \mathrm{U} \mathrm{CIP}$ at $37^{\circ} \mathrm{C}$ for $60 \mathrm{~min}$. CIP activity was then inactivated by addition of $200 \mu \mathrm{M}$ sodium orthovanadate. For subsequent in vitro ubiquitylation, $0.1 \mu \mathrm{M}+/$-CIP-treated His-Eps8 were incubated together with $75 \mu \mathrm{M}$ His-Ubiquitin, $170 \mathrm{nM}$ Ube1, $1 \mu \mathrm{M}$ UbcH5b, $150 \mathrm{nM} \mathrm{SCF}{ }^{\mathrm{Fbxw} 5}$, and $5 \mathrm{mM} \mathrm{ATP}$ at $30^{\circ} \mathrm{C}$ for 30 and $60 \mathrm{~min}$. In vitro reactions were separated on $8 \%$ gels and analyzed by $\alpha$-Eps8 western blotting (depicted in B)). 
Since we considered CIP-treatment-resistant phosphorylations in Eps8 very unlikely and since we were unable to detect phosphorylation sites in CIP-treated Eps8 samples by mass spectrometry (see below, result section 4.4, page 104), we concluded from these findings that $\mathrm{SCF}^{\mathrm{Fbxw5}}$ does not require Eps8 phosphorylation for ubiquitylation.
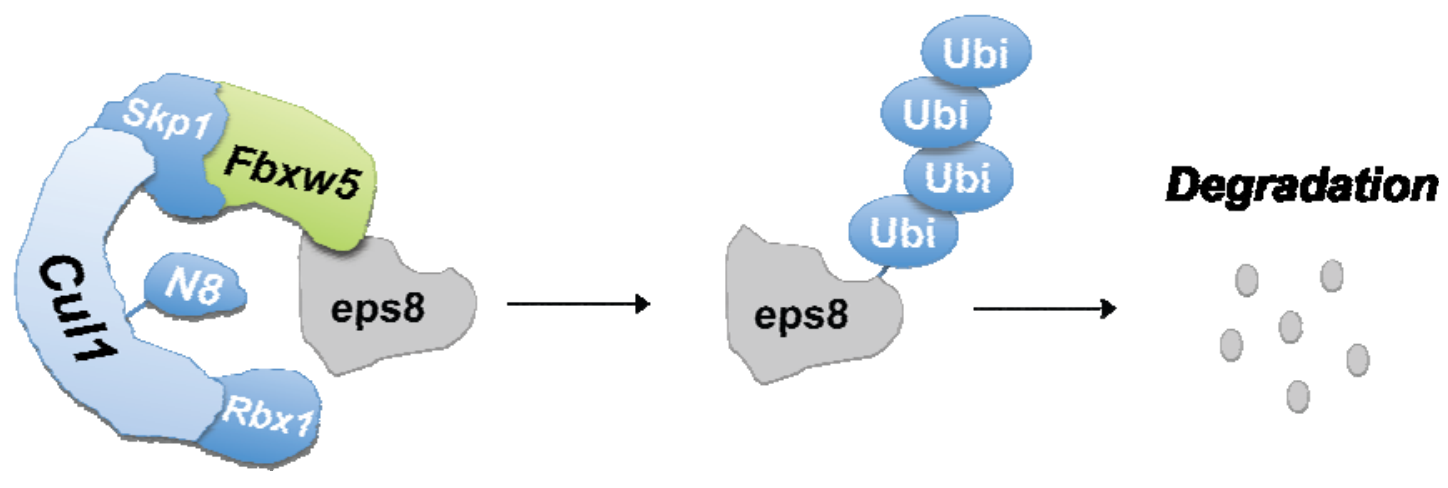

Figure 23: Current working model: $\mathrm{SCF}^{\mathrm{Fbxw5}}$ mediates the ubiquitylation of $\mathrm{Eps} 8$ in vitro and Fbxw5 contributes to Eps8 degradation in vivo.

Taking all in vivo and in vitro data on Fbxw5 and Eps8 together, the following working model can be postulated (Figure 23): Fbxw5 recognizes endogenous Eps8 and mediates its ubiquitylation in context of the SCF complex in vitro. In contrast to all other so far characterized proteins of the Fbxw family, Fbxw5 does not seem to require phosphorylations to recognize its target Eps8 (for further details see discussion, section 1.2, page 110). In cells, this ubiquitylation event is most likely involved in the degradation of Eps8 and involves so far unknown signals (for further details see discussion, section 2, page 114). 


\section{Nek9 phosphorylates Eps8 and inhibits its Fbxw5- dependent ubiquitylation}

The NIMA-related kinase 9 (Nek9) was originally described to be a regulator of mitosis (reviewed in (O'Regan, Blot, and Fry 2007)) and was recently identified to contribute to the control of macroautophagy (Behrends et al. 2010). Initial in vitro ubiquitylation and siRNA experiments conducted under similar conditions as for Eps8 provided no evidence for Fbxw5-mediated ubiquitylation and / or degradation of Nek9 (data not shown). Yet, Nek9 - together with Eps8 - was present in endogenous Fbxw5 immunoprecipitates (Figure 14, page 83). Hence, we next considered the possibility that Nek9 might regulate Fbxw5 or Eps8 via phosphorylation.

\subsection{Nek9 interacts with and phosphorylates Eps8}

Endogenous Nek9 immunoprecipitates contain Eps8

To collect more evidence for the hypothesis that Nek9 may regulate Eps8 or Fbxw5, I first wanted to characterize how Fbxw5, Nek9, and Eps8 interact. Knowing that Eps8 and Nek9 co-purify with Fbxw5 (Figure 14), I performed coimmunoprecipitation experiments from HeLa suspension cells using mouse $\alpha-$ Eps8 monoclonal and goat $\alpha$-Nek9 polyclonal antibodies followed by western blot analysis (Figure 24). Compared to the goat lgG control, a significant and specific amount of Eps8 co-purified with Nek9 (Figure 24A). In addition, very small, yet specific amounts of Fbxw5 could be detected in the IP fraction suggesting that all three proteins are present in a complex in vivo and interact with each other directly or indirectly. While an even smaller cellular fraction of Fbxw5 co-purified with Eps8, Nek9 was not detectable in Eps8 IPs (Figure 24B). There might be several reasons for this, including that Eps8 might be much more abundant in the cell than Nek9 and only a small percentage of Eps8 interacts with Nek9 at a given time point. The antibodies used in the IPs only captured a small percentage of cellular Eps8 and the amount of co-purifying Nek9 might just be too low for western blot detection. Alternatively, the epitope of the monoclonal $\alpha$-Eps 8 
antibodies might be located within the Eps8-Nek9 interaction surface, thereby interfering with binding.

While the possibility that the proteins interact with each other in distinct complexes could not be excluded, these data in combination with endogenous Fbxw5 immunoprecipitation experiments (Figure 14, page 83) do suggest the existence of an Fbxw5-Eps8-Nek9 complex in vivo.

A)

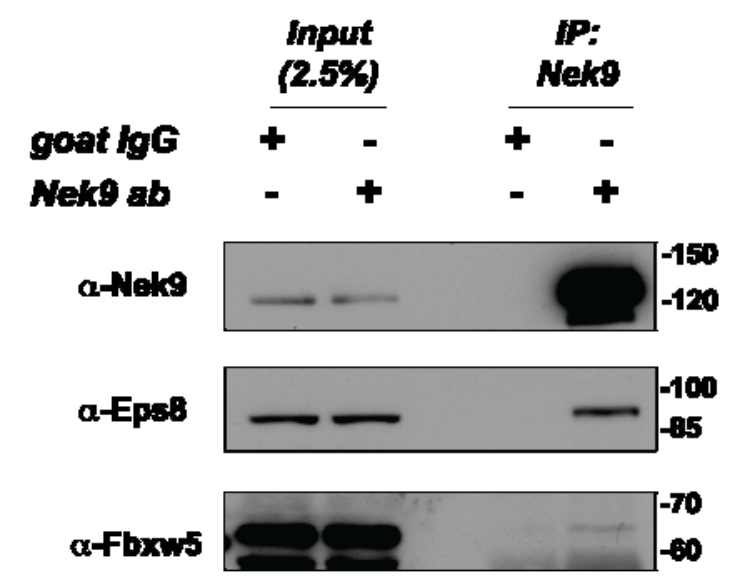

B)

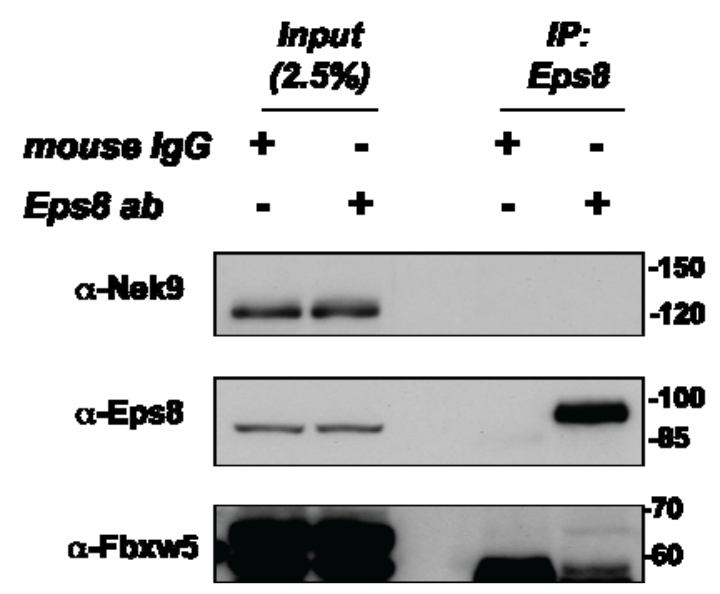

Figure 24: Eps8 co-purifies with Nek9 from HeLa suspension cells. A) $10 \mu \mathrm{g} \alpha$-Nek9 goat polyclonal antibodies were used to precipitate Nek9 from HeLa cell lysates (prepared from approximately $1 \times 10^{8}$ cells). In a control IP from the same amount of cells, goat IgGs were employed. B) IP was conducted as in A) but using $5 \mu \mathrm{g} \alpha$-Eps8 mouse monoclonal antibodies to precipitate Eps8 and mouse IgGs as control.

Phosphatase-treated Eps8 is an efficient target for in vitro phosphorylation by Nek9

To test whether Eps8 or Fbxw5 are targets for phosphorylation by Nek9, I next performed in vitro kinase assays. For this, HA-tagged Nek9 was transiently expressed in 293T cells and purified via $\alpha-\mathrm{HA}$ immunoprecipitation followed by HA peptide elution. Different amounts of these immunoprecipitates were then used to test their kinase activity towards recombinant Eps8 and Fbxw5/Skp1 complex. In case of Eps8, a treatment with calf intestine phosphatase (CIP) preceded the in vitro phosphorylation reactions for efficient removal of phosphorylations of Eps8 originating from its purification from SF9 cells (Figure 21). After incubation of HA-Nek9 immunoprecipitates with $\mathrm{mFbxw} 5 / \mathrm{Skp} 1$ complex or CIP-treated Eps8 in the absence and presence of ATP, reactions were 
analyzed by immunoblotting. Compared to the reaction containing no ATP, Eps8 was dramatically up-shifted in the SDS gel with increasing amounts of HA-Nek9, while such a shift could not be observed for Fbxw5 (Figure 25B). These observations demonstrated that Eps8 is phosphorylated in the presence of HANek9 IPs in vitro; hence Eps8 seems to be a direct interactor and substrate of Nek9. To ultimately prove this claim and to exclude contributions of other kinases co-purifying with Nek9 in the $\alpha-\mathrm{HA}$ IP, a catalytic mutant of Nek9 needs to be included as control in future experiments. In respect to Fbxw5, further kinase assays employing $\mathrm{P}^{32}$-labeled ATP are required to obtain conclusive results on whether it is a Nek9 substrate or not.

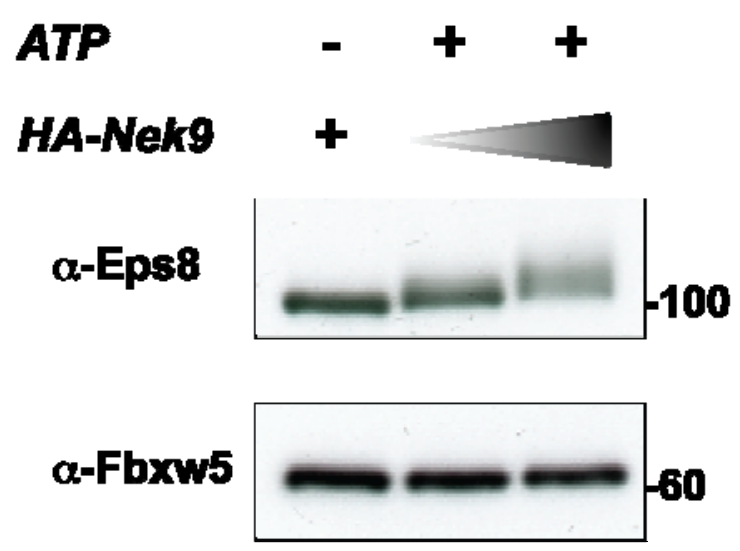

Figure 25: Nek9 phosphorylates Eps8 in vitro. In vitro kinase assays with immunopurified HA-Nek9. $2.5 \mu \mathrm{M}$ CIP-treated mouse full-length His-Eps8 or $2.5 \mu \mathrm{M}$ $\mathrm{mFbxw5/Skp1} \mathrm{complex} \mathrm{were} \mathrm{incubated} \mathrm{in} \mathrm{SAB}$ buffer with different amounts of HA-Nek9 in the absence and presence of $5 \mathrm{mM} \mathrm{ATP}$ at $30^{\circ} \mathrm{C}$ for $60 \mathrm{~min}$.

\subsection{Nek9 phosphorylation of Eps8 inhibits its Fbxw5-mediated ubiquitylation}

The finding that Eps8 can be efficiently phosphorylated in vitro in the presence of Nek9 immunoprecipitates raised the intriguing possibility that Fbxw5-mediated ubiquitylation of Eps8 might be influenced and regulated by Nek9-dependent phosphorylation. Hence, we next performed in vitro experiments exploring the impact of the phosphorylation status of Eps8 on its $\mathrm{SCF}^{\mathrm{Fbxw5}}$-mediated ubiquitylation.

For this, pools of Eps8 with different phosphorylation states were generated by successive phosphatase and Nek9 treatment of Eps8 purified from SF9 cells (Figure 26A). This way, fractions of Eps8 containing only phosphorylations originating from SF9 cells (- phosphatase / - Nek9), containing phosphorylations originating from SF9 cells and Nek9 treatment (- phosphatase / + Nek9), 
containing only phosphorylations from Nek9 treatment (+ phosphatase / + Nek9), and fractions devoid of phosphorylations (+ phosphatase / - Nek9) were obtained. These different pools of Eps8 were then compared in $\mathrm{SCF}^{\mathrm{Fbxw5}}$-dependent ubiquitylation in time course experiments (Figure 26B). As observed before, phosphorylation of Eps8 was not required for efficient ubiquitylation by $\mathrm{SCF}^{\mathrm{Fbxw} 5}$, since fractions of Eps8 devoid of phosphorylations were modified in a similar manner as Eps8 fractions carrying phosphorylations from SF9 cells (lanes 7-9 vs. lanes 1-3, Figure 26B). In contrast, incubation of dephosphorylated Eps8 with Nek9 prior to ubiquitylation reactions resulted in an almost complete blockage of Ubiquitin conjugation to Eps8 (lanes 7-9 vs. lanes 10-12, Figure 26B). Such a dramatic effect was not observed for Eps8 fractions that were incubated with Nek9 without prior removal of phosphorylations (lanes 4-6 vs. lanes 10-12, Figure 26B). Only a slight inhibition of $\mathrm{SCF}^{\mathrm{Fbxw5}}$-mediated ubiquitylation could be detected for this Eps8 pool.

A)

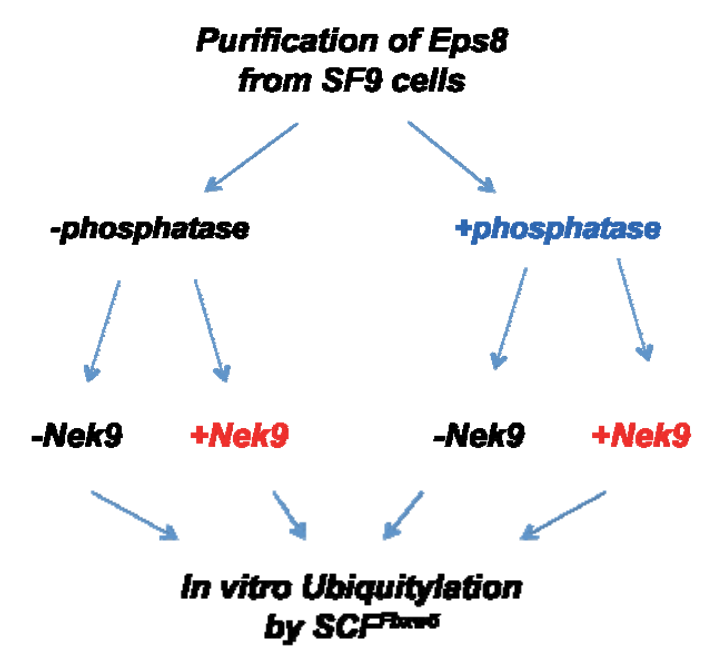

B)

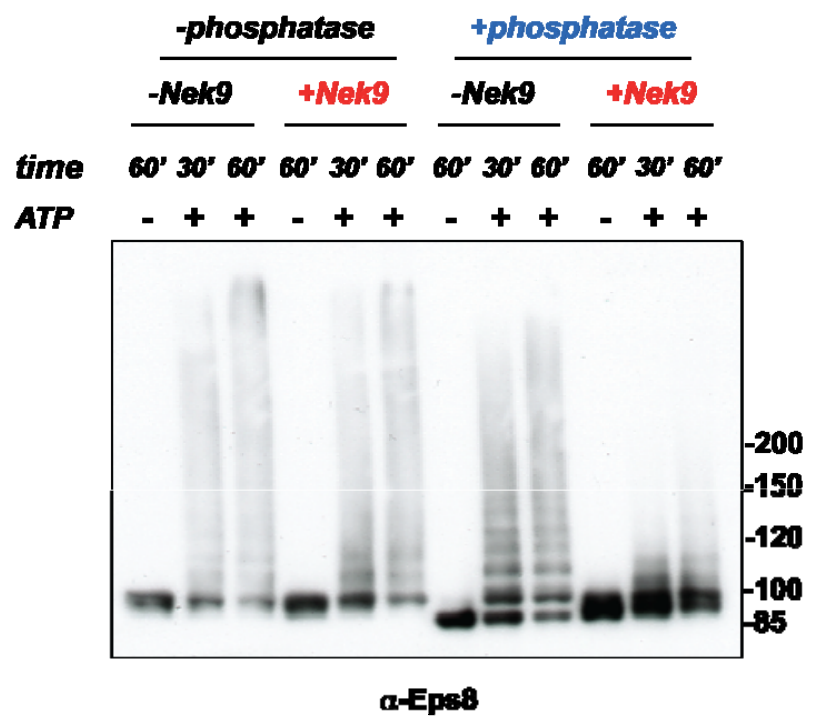

Figure 26: Nek9 phosphorylation of Eps8 inhibits its Fbxw5-mediated ubiquitylation. A) Experimental flow chart. $5 \mu \mathrm{M}$ mouse full length His-Eps8 purified from SF9 cells were subjected to dephosphorylation by incubation with (+ phosphatase) or without (- phosphatase) $10 \mathrm{U}$ CIP at $37^{\circ} \mathrm{C}$ for $60 \mathrm{~min}$ followed by inactivation of CIP by addition of $200 \mu \mathrm{M}$ sodium orthovanadate. For subsequent in vitro kinase assays, $2.5 \mu \mathrm{M}$ His-Eps8 (+/- prior phosphatase-treatment) were incubated in the absence and presence of HA-Nek9 at $30^{\circ} \mathrm{C}$ for 60 min. Finally, for ubiquitylation of Eps8 fractions with different phosphorylation states, $0.1 \mu \mathrm{M}+/$-CIP-treated and +/- Nek9-treated His-Eps8 were incubated together with $75 \mu \mathrm{M}$ His-Ubiquitin, $170 \mathrm{nM}$ Ube1, $1 \mu \mathrm{M}$ UbcH5b, $150 \mathrm{nM}$ $\mathrm{SCF}^{\mathrm{Fbxw} 5}$, and $5 \mathrm{mM} \mathrm{ATP}$ at $30^{\circ} \mathrm{C}$ for 30 and $60 \mathrm{~min}$. In vitro reactions were separated on $8 \%$ gels and analyzed by $\alpha$-Eps8 western blotting (depicted in $\mathbf{B}$ )). 
These data suggested that Nek9 can negatively regulate $\mathrm{SCF}^{\mathrm{Fbxw5}}$-mediated ubiquitylation of Eps8 via phosphorylation of residues within Eps8 required for Fbxw5 recognition. Other mechanisms of inhibition, such as a) steric hindrance due to binding of Nek9 to Fbxw5 or Eps8, b) competition of Nek9 as substrate for $\mathrm{SCF}^{\mathrm{Fbxw5}}$, or c) Nek9-mediated phosphorylation of / binding of Nek9 to components of the ubiquitylation machinery could be excluded. Reasons for this were that the in vitro reactions contained an approximate 10-fold molar excess of Eps8 and Fbxw5 over Nek9 (assuming a Nek9 concentration of $50 \mathrm{ng} / \mu \mathrm{L}$ ), while the ubiquitylation inhibition was almost complete. Furthermore, no evidence for Fbxw5-mediated ubiquitylation of Nek9 could be obtained within this thesis (data not shown). Most importantly, ubiquitylation was only slightly inhibited when Eps8 was incubated with Nek9 without prior removal of phosphorylations. This suggested that most of the cellular Eps8 is phosphorylated in such a way that Nek9 cannot recognize and further modify it. This observation clearly indicated that the blockage of ubiquitylation in phosphatase- and Nek9-treated Eps8 had to be directly due to HA-Nek9 IP-dependent phosphorlyation.

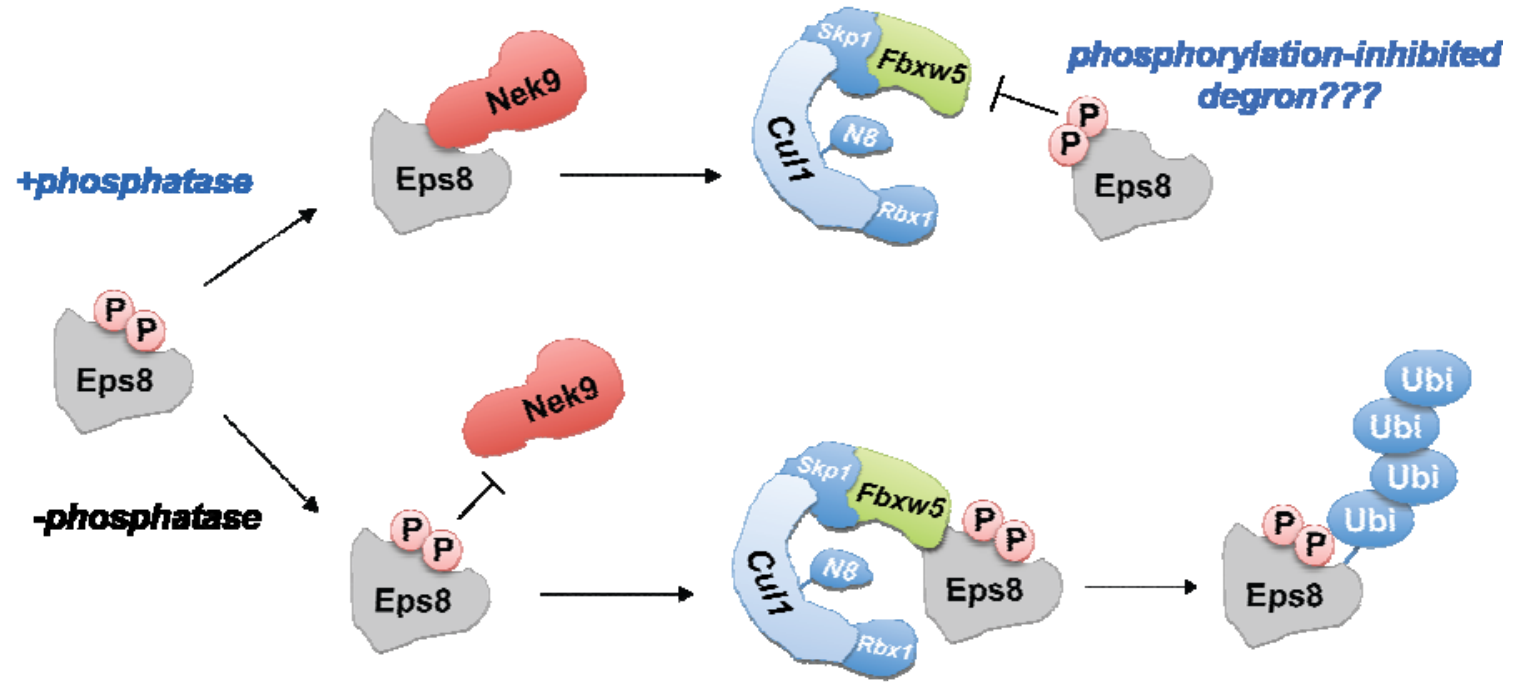

Figure 27: Current working model: $\mathrm{SCF}^{\mathrm{Fbxw5}}$-mediated ubiquitylation of Eps8 is negatively regulated by Nek9 phosphorylation. Most of the cellular pool of Eps8 is phosphorylated in such a way that it is inaccessible for Nek9 recognition and phosphorylation and therefore readily ubiquitylated by $\mathrm{SCF}^{\mathrm{Fbxw5}}$ in vitro. If, however, these inhibitory phosphorylations are removed by phosphatase treatment, Nek9 can efficiently phosphorylate Eps8 at residues that block recognition by $\mathrm{Fbxw} 5$, thereby inhibiting $\mathrm{SCF}^{\mathrm{Fbxw} 5}$-mediated ubiquitylation. 
Taken together, the findings of these in vitro assays demonstrated that Nek9 can indeed regulate $\mathrm{SCF}^{\mathrm{Fbxw}}$-mediated ubiquitylation of Eps8 and allowed to postulate a model (Figure 27), in which Nek9-dependent phosphorylation of Eps8 inhibits Fbxw5-mediated ubiquitylation. Intriguingly, this is opposite to the current view of how Fbw proteins recognize their targets and, to our knowledge, the first evidence pointing towards a phosphorylation-inhibited degron for an F-box protein.

\subsection{Nek9 might regulate Eps8 levels in vivo}

Until this point, the hypothesis that Nek9 might negatively regulate $\mathrm{SCF}^{\mathrm{Fbxw5}}$ dependent ubiquitylation (Figure 27) had been primarily deduced from in vitro observations. To challenge this hypothesis and to obtain some in vivo evidence for our model, I next preformed several cell biological experiments in HeLa cells.

\subsubsection{Phosphatase treatment of HeLa cytosol increases the amount of co-} purifying Eps8 in Nek9 IPs

The observation that Nek9 inhibited Eps8 ubiquitylation only after removal of preexisiting phosphorylations suggested that the predominant pool of Eps8 within cells carries phosphorylations that prevent Nek9 binding. One prediction from this model would be that phosphatase treatment of HeLa cytosol prior to Nek9 IP should increase the fraction of co-purifying Eps8. To test this hypothesis, I prepared cytosol from HeLa CSH suspension cells and incubated it in the absence and presence of phosphatase, followed by immunoprecipiation of endogenous Nek9 and investigation of co-purifying Eps8 and Fbxw5 fractions by immunoblotting (Figure 28A). As judged from the Ponceau $S$ stain of the nitrocellulose membrane and the $\alpha$-Nek9 western blot, Nek9 was immunoprecipitated from phosphatase-treated and -untreated cytosol in a specific and equally efficient manner (Figure 28B). While significantly more Eps8 copurified with Nek9 upon phosphatase treatment, Fbxw5 amounts bound to Nek9 were equal under both IP conditions. Of note, more interaction partners could be detected on the Ponceau-stained membrane for Nek9 IPs from mock-treated compared to the phosphatase-treated cytosol. 
A)

\section{HeLa cytosol from CSH suspension cells}
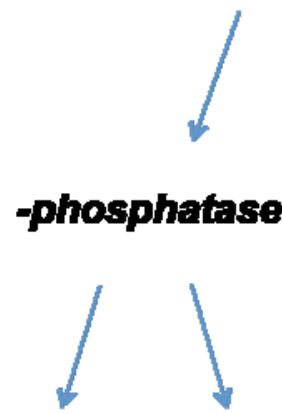

IgGIP Nek9IP IgGIP NekgIP
B)

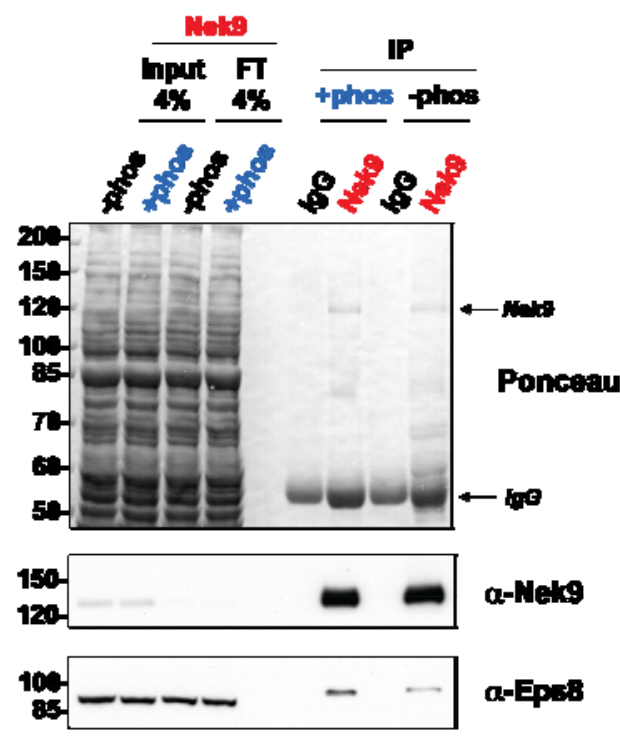

Figure 28: Phosphatase treatment of HeLa cytosol increases the amount of Eps8 in endogenous Nek9 immunoprecipitates. A) Experimental scheme B) Cytosol was prepared from $4 \times 10^{8} \mathrm{HeLa}$ CSH cells and was split into two fractions; one being incubated in the presence, the other one in the absence of CIP at $30^{\circ} \mathrm{C}$ for $1 \mathrm{~h}$. From these +/- phosphatasetreated cytosolic fractions, Nek9 IPs were performed using goat IgGs as control. Input, flow through (FT), and immunoprecipitated (IP) fractions were then analyzed by Ponceau $S$ staining and western blotting.

In summary, these findings suggested that cellular Eps8 indeed carries phosphorylations that prevent interaction with Nek9. The inhibitory modifications can be partially removed by phosphatase treatment resulting in a larger fraction of co-purifying Eps8 in endogenous Nek9 IPs.

4.3.2. Overexpression of constitutively active Nek9 stabilizes Eps8 levels in HeLa cells

Considering that Fbxw5 contributed to Eps8 degradation in vivo (Figure 17, page 88) and Nek9-mediated Eps8 phosphorylation inhibited its $\mathrm{SCF}^{\mathrm{Fbxw5}}$-dependent ubiquitylation in vitro, we reasoned that Nek9 should contribute to Eps8 stability in cells. 


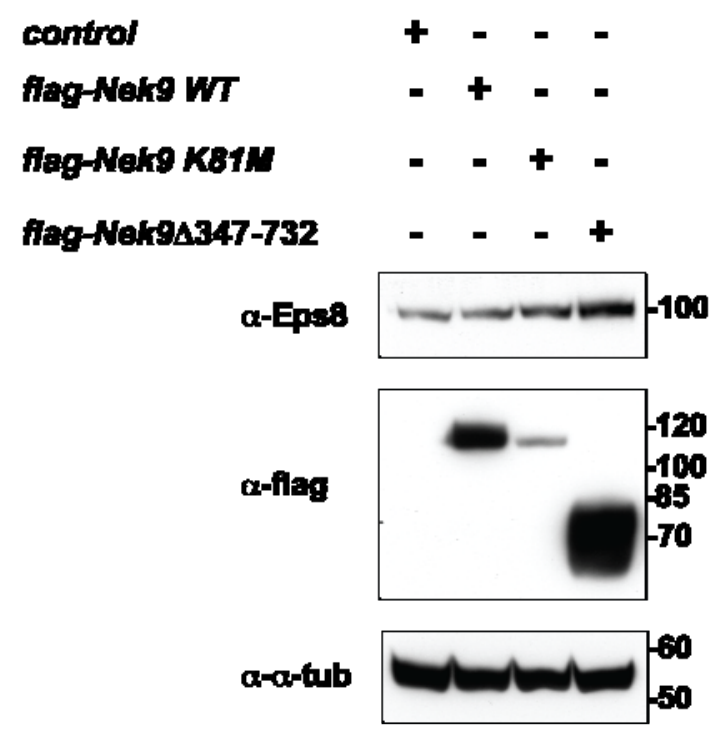

Figure 29: Overexpression of constitutively active Nek9 stabilizes Eps8 levels in HeLa cells. $50-60 \%$ cionfluent HeLa cells were transfected with pCDNA3.1-flag (control), pCDNA3.1-flag-Nek9 wild type (WT), pCDNA3.1flag-Nek9 K81M (kinase dead mutant), or pCDNA3.1-flag-Nek9 3477-732 (constitutively active mutant) using JetPRIME ${ }^{\mathrm{TM}}$ reagent. $30 \mathrm{~h}$ post transfection, cells were harvested by direct lysis in 2xSDS sample buffer and analyzed by SDS PAGE followed by immunoblotting with the indicated antibodies. An $\alpha-\alpha$-tubulin immunoblot $(\alpha$-tub) served as loading control.

To test this hypothesis, I transfected HeLa cells with plasmids encoding flagtagged wild type Nek9 (flag-Nek9 WT), a catalytically inactive mutant of Nek9 (flag-Nek9 K81M), and a constitutively active deletion mutant of Nek9 (flagNek9 $4347-732$ ) and incubated the cells for $30 \mathrm{~h}$. Cells were subjected to direct lysis in 2xSDS sample buffer and lysates were analyzed by SDS PAGE and immunoblotting (Figure 29). Comparing cell lysates from cells transfected with control plasmids with those transfected with flag-Nek9 $4347-732$, a significant inrcrease of Eps8 levels could be detected (Figure 29 lanes 1 vs. 4). In light of the greatly varying expression levels of the different flag-Nek9 versions, it was unfortunately not possible to make comparisons amongst cells transfected with the different flag-Nek9 versions or to directly conclude that the kinase activity of Nek9 is required for the effect on Eps8. Since these variances had also been observed by others (Belham et al. 2003), they can be most likely explained by different intrinsic properties of the constructs / proteins rather than technical issues during transfection.

In summary and consistent with our model, these data suggested that Nek9 can indeed stabilize Eps8 levels in cells. 


\subsection{Mass spectrometry analysis reveals Nek9-dependent phosphorylation sites in Eps8}

Substrate recognition of all so far characterized F-box proteins of the Fbw class requires posttranslational modification within a short amino acid degradation sequence called degron (Cardozo and Pagano 2004). Surprisingly, our in vitro ubiquitylation experiments of Eps8 suggested that this rule does not apply to Fbxw5. On the contrary, Nek9 phosphorylation of Eps8 inhibited its Fbxw5mediated ubiquitylation and the transfection experiment in Figure 29 suggested that this might have a functional role in vivo. To further investigate this finding at a molecular level, we performed mass spectrometry analysis to identify the phosphorylation sites within Eps8 that block its ubiquitylation.

A)

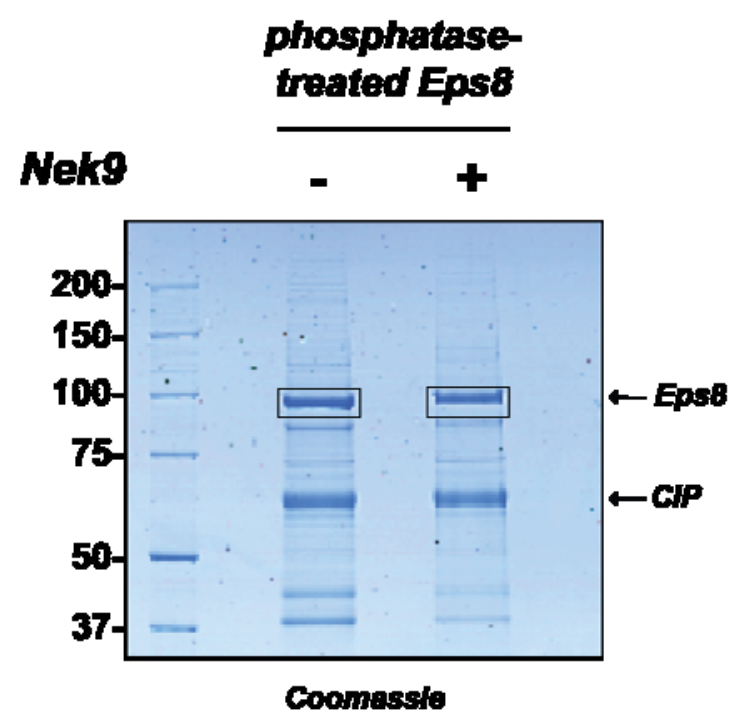

B) Eps8 phosphopeptides specifically identified upon Nokg treatment

\begin{tabular}{|c|c|c|}
\hline peptide sequence & $\begin{array}{c}\text { modified } \\
\text { residue }\end{array}$ & score \\
\hline ARDSVSŞVSDVSQY & Ser58 & 46,88 \\
\hline 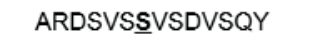 & Ser58 & 17,75 \\
\hline RVEHLTIF & Thr72 & 23,65 \\
\hline RVEHLTITF & Thr72 & 17,17 \\
\hline KSTPNHQVDRNY & Ser514 & 26,52 \\
\hline KSIPNHQVDRNY & Thr515 & 31,74 \\
\hline KSIPNHQVDRNY & Thr515 & 25,15 \\
\hline DAVKIQPKKY & Thr529 & 24,07 \\
\hline IIGRSAAQRKF & Thr698 & 19,3 \\
\hline THIIQKQRTEY & Thr698 & 25,99 \\
\hline SLNKDELR్VCPEGARVF & Ser764 & 35,75 \\
\hline SLNKDELR $\underline{S} V C P E G A R V F$ & Ser764 & 19,62 \\
\hline
\end{tabular}

Figure 30: Mass spectrometry identification of Nek9-dependent phosphorylation sites within Eps8. A) Coomassie staining of $5 \mu \mathrm{g}$ phosphatase-treated Eps8 and $5 \mu \mathrm{g}$ phosphataseand Nek9-treated Eps8 (generated as described in material and methods) used for mass spectrometry analysis B) Table summarizing phosphopeptides specifically identified in phosphatase- and Nek9-treated Eps8 fractions. In bold and underlined are residues that are phosphorylated. Listed are also the peptide scores. Mass spectrometry analysis was carried out by the lab of Henning Urlaub, MPI-BPC in Göttingen.

For this, in vitro kinase assays with Eps8 and Nek9 were up-scaled to generate 5 $\mu \mathrm{g}$ phosphatase-treated Eps8 and $5 \mu \mathrm{g}$ phosphatase- and Nek9-treated Eps8 that 
were separated on SDS Page and were Coomassie stained (Figure 30A). As observed before, the Eps8 fraction treated with Nek9 exhibited a decreased electrophoretic mobility compared to the untreated fraction, indicating that Nek9 had quantitatively phosphorylated Eps8. Bands for both pools of Eps8 were then cut out, were in-gel digested with chymotrypsin, and isolated peptides were analyzed by ESI-Trap MS by the lab of Henning Urlaub at the MPI-BPC in Göttingen (as described in materials and methods, section 2.2.12, page 69). Comparing the lists of Eps8 peptides from the different Eps8 fractions, the following observations were made: a) in both samples the same amount of Eps8 peptides covering approximately $60 \%$ of the protein sequence were detected; b) only the Nek9-treated Eps8 fraction contained phosphopeptides, which are listed in Figure 30B.

These data clearly demonstrated that Nek9 can phosphorylate Eps8 in vitro at seven distinct serine and threonine residues located at various regions in its sequence (Figure 31). Database and literature searches revealed that these residues are novel phosphorylation sites of Eps8 that have not been described yet. A likely explanation for this is the putative low abundance of these modifications in cells. Under normal growth conditions, only a small pool of Eps8 can be recognized by Nek9 (Figure 28), suggesting that very little Eps 8 molecules actually carry Nek9-dependent phosphorylations. The fact that no phosphopeptide was identified in the CIP-treated Eps8 fraction further supported our hypothesis that Fbxw5 indeed recognizes Eps8 in a phosphorylationindependent manner.

While it cannot be excluded that there are further Nek9-dependent phosphorylation sites located within the Eps8 sequence not covered by mass spectrometry, the already identified residues are a good starting point for future studies dedicated to identify the interaction site of Fbxw5 within Eps8 and to collect further evidence for a phosphorylation-inhibited degron. Obvious future steps include the generation of Eps8 peptides flanking the identified phosphoresidues to test their ability to compete with the $\mathrm{SCF}^{\mathrm{Fbxw5}}$-mediated in vitro ubiquitylation of Eps8. Alternatively, phospho-mimicking and phospho-deficient Eps8 mutants will be generated and tested for inhibition in $\mathrm{SCF}^{\mathrm{Fbxw5}}{ }_{-}$-mediated 
ubiquitylation, Nek9 binding, and Nek9-depednent inhibition of ubiquitylation, respectively.

\section{Mouse Eps8 sequence}

\begin{tabular}{|c|c|c|c|c|c|}
\hline 10 & 20 & 30 & 40 & 50 & 60 \\
\hline Metwoshs: & GrovrPagn & Grasa & 2 MDRE & ARAIYEORKM & YARDS VSS \\
\hline 70 & 80 & 90 & 100 & 110 & 120 \\
\hline VSQFRVEMI, & TIFVIDRROA & Nrтyostax & LKLIDAKEKY & MrCDNI LQWD & 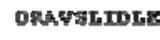 \\
\hline 130 & 250 & 150 & 160 & 170 & 100 \\
\hline HUELEGPSW & 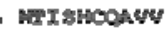 & aACsYDSTIA & Isckereroesk & PDLHLFOCDE & GKAME I SEDI \\
\hline 190 & 200 & 210 & 220 & 230 & 240 \\
\hline IAAISDSEGGG & HOKRRPEALR & MIAEADEGIP & PRERAPAPUE & PGTVROVDNR & SAUAAHFAAEA \\
\hline 250 & 260 & 270 & 280 & 290 & 300 \\
\hline DQGDFERPR & CHHBOESTPZ & ImLAA: & OIHEIIIDDI & BFFITKLOKA & GLSRR \\
\hline 310 & 320 & 330 & 340 & 350 & 360 \\
\hline RSIRKSIKRIKG & GEGULPLRA & REREDEFW & CFORFRHEFH & ILAKLKBHTQ & WEAAGDWWTF \\
\hline 370 & 390 & 390 & 400 & 410 & 420 \\
\hline expo & PEGPELAA9 & TrRDT & VDFIMYTATA & EERKINMIEG & STRAEm \\
\hline 430 & 440 & 450 & 460 & 970 & 480 \\
\hline CEEY & PRFRMEMEge & IGAPTE & THAESY & AMASHQRNQD & 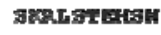 \\
\hline 490 & 500 & 510 & 520 & 530 & 540 \\
\hline$A D C X$ & AY\$S: & CEAAM & mugy & DRMYY & DFRYARSAXYD \\
\hline 550 & 560 & 570 & 520 & 590 & 200 \\
\hline FaAR月SSELS & WKDDVLers & mos & sepus & MNILDIMAR? & 8HCUARADS? \\
\hline 610 & 620 & 630 & 640 & 650 & 650 \\
\hline YTIYIOKQET & EYGLRAAOTE & SARSEREY & PUPUELPES4 & PAPUSUPWU & Ao4houss: \\
\hline 670 & 600 & 600 & 700 & 720 & 720 \\
\hline IVRD & RYKOLEVD & JEREWQ & RITIO & KFUU & pase \\
\hline 730 & 740 & 750 & 760 & 770 & 780 \\
\hline 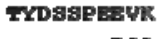 & & & & & \\
\hline 190 & 0 & 10 & 820 & & \\
\hline
\end{tabular}

\section{Phosphopeptides identifled} via mass spec

Nek9 phosphorylation sites

publishedphosphorylation sites

Figure 31: Overview of known and newly identified phosphorylation sites within Eps8. Eps8 has been shown to interact with several kinases including Akt1, Src and MAPK. In large-scale mass spectrometry screens, several phoshporylations sites in Eps8 (highlighted in red) have been identified. In particular, it has recently been suggested that MAPK-dependent phosphorylation of Ser624 and Thr628 releases Eps8 from actin structures in hippocampal neurons (Menna et al. 2009). Mass spectrometry analysis of Nek9-treated Eps8 fractions by the lab of Henning Urlaub at the MPI-BPC in Göttingen revealed novel, so far unidentified phosphorylation sites in Eps8 marked in red. Identified Eps8 phosphopeptides are highlighted in bold. 


\section{DISCUSSION}

Despite the pivotal role of selelcted F-box proteins in diverse cellular processes such as cell cycle regulation, signal transduction, and gene expression (Petroski and Deshaies 2005), surprisingly little is known about the biological functions of most members of this protein family. Up to date, for only approximately 20 of the 69 mammalian F-box substrates are known (Skaar et al. 2009).

Within this work, I investigated one of these rather uncharacterized F-box proteins, Fbxw5, and made some intriguing findings in respect to its mechanistic and functional properties. In the following section some important details and interesting aspects arising from the presented results will be discussed.

\section{Fbxw5 - a F-box protein with unconventional features}

\subsection{Fbxw5 - a bifunctional substrate recognition subunit}

Since the discovery of the F-box domain within Fbxw5 and the demonstration of its association to Skp1 (Winston et al. 1999) and Cul1 (Dorrello et al. 2006) upon overexpression, the functional and mechanistic properties of Fbxw5 have remained rather enigmatic: despite the fact that overexpressed Fbxw5 can interact with Skp1 and Cul1 upon IP, there are to date no reports that unambiguously demonstrate the existence of functional $\mathrm{SCF}^{\mathrm{Fbxw5}}$ complexes in vivo. On the contrary, Fbxw5 was reported to function as a SRS in context of CRL4 complexes thereby not requiring its F-box domain. The data presented within this work demonstrate for the first time that Fbwx5 endogenously assembles into and functions via SCF complexes. Consistent with previous work from other groups (He et al. 2006; Hu et al. 2008), I found that Fbxw5 also associates with components of Cul4A-based complexes - yet, to a significantly smaller extent than to those of Cul1-based complexes under normal growth conditions of mammalian tissue culture cells (Figure 9, page 74). Hence, Fbxw5 seems to use both, Cul1- and Cul4A-based scaffolds to carry out its function as substrate recognition subunit. What is the advantage of such a bifunctionality? Since we currently don't know, whether the two types of Fbxw5-containing CRL 
complexes are mutually exclusive or whether a Cul1-Skp1-Fbxw5-DDB1-Cul4A complex could also form in vivo, there are at least two answers to this question. In one scenario, Fbxw5 might assemble either into CRL1 or into CRL4 complexes and depending on which complex it is integrated into, it targets distinct sets of substrates (Figure 32A). Thus, the scaffold of the CRL would contribute to substrate specificity. Evidence in favor of such a model is the finding that Fbxw5 does not seem to require its F-box domain and hence also not the context of an SCF complex for its functions in TSC2 degradation and TAK1 regulation (Hu et al. 2008; Minoda et al. 2009). Conversely, the ubiquitylation of Eps8 at least in vitro requires the F-box domain of Fbxw5 and therefore the integrity of the SCF complex (Figure 18, page 90). Taken together, these observations suggest the existence of functionally distinct $\mathrm{SCF}^{\mathrm{Fbxw} 5}$ and $\mathrm{CRL} 4^{\mathrm{Fbxw} 5}$ complexes and raise the intriguing possibility of a novel type of regulation among the superfamily of CRLs: substrate specificity regulation via scaffold switching (Figure $32 \mathrm{~A}$ ). In this context and considering the predominance of $\mathrm{SCF}^{\mathrm{Fbxw5}}$ complexes under normal cellular growth conditions, it is attractive to speculate that the equilibrium between $\mathrm{SCF}^{\mathrm{Fbxw} 5}$ and $\mathrm{CRL} 4^{\mathrm{Fbxw} 5}$ complexes within the cell might be regulated via certain stimuli. These signals could alter the sub-cellular localization of one or several of the involved $\mathrm{CRL}$ components, thereby promoting the association of Fbxw5 into one of the two types of CRL complexes. At least for DDB1, such a signal-induced change of localization has been described. DDB1, which under normal conditions localizes to the cytoplasm, translocates into the nucleus upon UV damage (Liu et al. 2000).

In a second scenario, Fbxw5 might associate to Cul1- and Cul4-based CRLs at the same time, thereby forming an E3 ligase complex containing two RING domains for E2 binding (Figure 32B). To date, several E3 ligases and E3 ligase complexes have been shown to function via two RING domains. For example, Parkin contains two RING finger domains, both of which are indispensible for its E3 activity (Imai, Soda, and Takahashi 2000). BRCA1 and BARD1, both of which contain a single RING domain, dimerize to exert E3 activity (Hashizume et al. 2001). In addition, it has been observed that several SCF ligases dimerize via their F-box protein to form homo- and heterodimers that are thought to be required for optimal E3 ligase activity (Merlet et al. 2009). The underlying 
mechanism of how dimerization promotes ubiquitylation (at least in the case of $\mathrm{SCF}^{\mathrm{Cdc} 4 / \mathrm{Fbxw7}}$ ) is to facilitate lysine acceptor site utilization by optimally positioning specific substrate lysine residues towards the E2 active site (Tang et al. 2007). In this context, one requirement of F-box protein-mediated dimerization is the presence of a small domain within the F-box protein, termed D-domain, which is located N-terminally to the F-box motif and seems to be essential for degradation of targets in vivo. Since Fbxw5 does not contain such a D-domain and my own data suggests that it cannot form homodimers upon overexpression in HEK 293T cells (data not shown), it is intriguing to speculate that the formation of mixed CRL1/4 ${ }^{\mathrm{Fbxw5}}$ complexes would be an alternative means to form a dimeric E3. Following along those lines, another F-box protein with no recognizable Ddomain, Fbxw8, seems to also have developed an alternative strategy for dimer formation. Tsunematsu and colleagues have demonstrated in vivo that Fbxw8 can interact with Skp1-Cul1-Rbx1 (via its F-box domain) and Cul7 (via its Cterminal portion) at the same time (Tsunematsu et al. 2006). Whether the

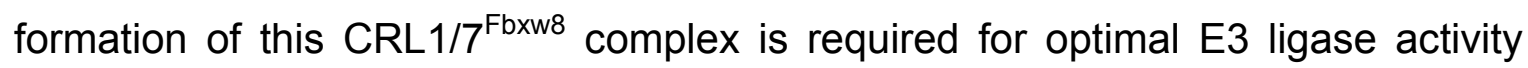
has yet to be determined.

Further experiments are required to test whether the specificity or activity of Fbxw5 is indeed regulated by scaffold switching or formation of heterodimeric CRL complexes, respectively, or whether Fbxw5 is subject to both types of regulation. One line of investigation would involve in vitro reconstitution of $\mathrm{CRL4}^{\mathrm{Fbxw5}}$ complexes and the comparison of their ubiquitylation properties towards TSC2 and Eps8 to those of SCF ${ }^{\mathrm{Fbxw} 5}$ complexes. Alternatively, one could test for interaction of Cul1 and Cul4A in co-immunoprecipitation experiments from cells. If such an interaction existed in an Fbxw5-dependent manner, next steps could include the in vitro reconstitution of such heterodimeric Fbxw5 complexes and comparison of their functional properties to those of $\mathrm{SCF}^{\mathrm{Fbxw5}}$ complexes in ubiquitylation assays. 
A)

\section{Substrate specificity for target A}

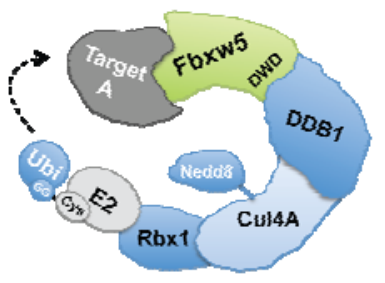

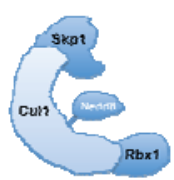

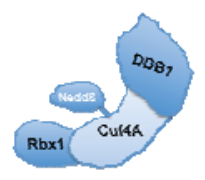

Substrate specificity for target B
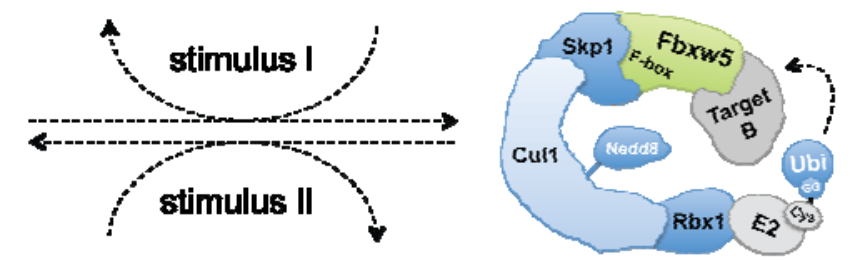

B)

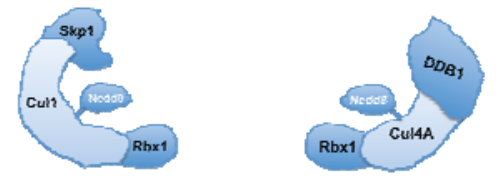

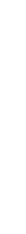


of Fbxw1 and Fbxw7 in complex with their cognate $\beta$-catenin and cyclinE phosphodegrons, respectively, general principles for phosphodegron binding by Fbxw proteins have been described (Wu et al. 2003; Hao et al. 2007). The WD40 repeats of both $\mathrm{Fbxw}$ proteins form a $\beta$-propeller structure, which has a narrow channel running through its middle. The $\beta$-catenin and cyclinE phosphodegrons are recognized on top of the $\beta$-propeller structures of Fbxw1 and Fbxw7, respectively, involving three conserved positions of the WD40 repeat (Figure 33). These structures underline the importance of the $\beta$-propeller fold for target recognition and they also give a molecular explanation of the exquisite specificity of the F-box proteins towards the phosphorylated form of their targets, since the phosphate groups within the degrons make the largest number of contacts with residues of the respective F-box protein.

Interestingly, in vitro ubiquitylation assays of Eps8 fractions with different phosphorylation states seemed to point towards a quite different recognition mode for Fbxw5 and Eps8 than anticipated from the canonical view described above: $\mathrm{SCF}^{\mathrm{Fbxw5}}$ did not significantly discriminate between fractions of phosphorylated Eps8 from SF9 or 293T cells and Eps8 pools, in which the phosphorylations had been removed by CIP-treatment (Figure 22). These observations suggest that Fbxw5 recognizes Eps8 in a phosphorylationindependent manner. Strikingly, as indicated by phosphorylation of Eps8 by Nek9 IPs and subsequent application of these Eps8 pools in ubiquitylation experiments, phosphorylation of certain residues within Eps8 completely blocked its $\mathrm{SCF}^{\mathrm{Fbxw5}}$ mediated ubiquitylation (Figure 26). This raises the intriguing possibility that for Fbxw5, phosphorylation is not a positive signal for substrate binding, but might rather inhibit substrate recognition - a regulatory mechanism exactly the opposite to the one described for all other Fbxw proteins characterized so far. Future studies are certainly required to investigate whether phosphorylation is indeed a means to negatively regulate Fbxw5-mediated ubiquitylation of Eps8 in vivo and what role Nek9 might play in this process (for further discussion see to the next chapters). 


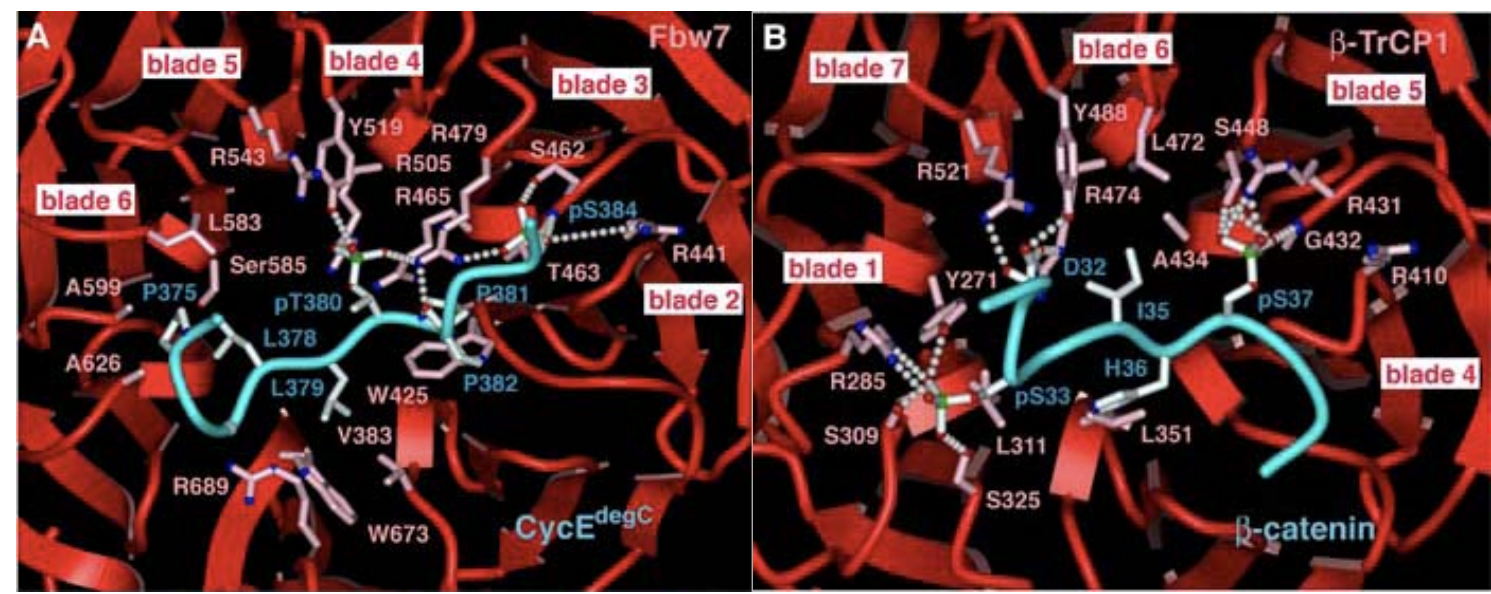

Figure 33: Phosphodegron-recognition by Fbxw7 and Fbxw1 ( $\beta$-TRCP1) is similar and requires residues at three conserved positions within the WD40 repeat. Picture was adapted from (Hao et al. 2007) and depicts Fbxw7 in complex with the cyclinE phosphodegron (A) and Fbxw1 ( $\beta$-TrCP1) in complex with the $\beta$-catenin phosphodegron (B). To illustrate the similar mode of phosphopeptide recognition by Fbxw7 and Fbxw1, the two complexes were superimposed by aligning blades 2-3-4-5-6 of the eight-bladed Fbxw7 on blades 4-5-6-7-1 of the seven-bladed $\beta-$ TrCP1. This roughly aligns the channel pocket and the two surface pockets in the two complexes. The previous comparison of $\beta$-TrCP1 with other WD40 protein-protein complexes (Wu et al. 2003) had shown that most WD40-peptide contacts are made by conserved positions on each blade (the second residue of the A strand, the residue immediately prior to the start of the A strand, and the residue immediately after the $B$ strand) and this is recapitulated in the Fbxw7-Cyclin $E^{\text {degron }}$ complex.

In summary, these observations suggest fundamental differences in how Fbxw5 binds its target Eps8 and how this recognition is regulated compared to known Fbxw proteins. In light of the fact that it is currently unclear whether the C-terminal part of Fbxw5 folds into a $\beta$-propeller structure (Figure 8 , page 31 ), it will be interesting to see whether these differences in the functional properties are also reflected in the structure of Fbxw5 compared to those of Fbxw1/7. To determine the structure of Fbxw5 and to elucidate how it interacts with Eps8, X-ray crystallography or NMR are certainly the methods of choice. Since such an approach might take time, a faster, biochemical way to get insights into the interaction site of Fbxw5 within Eps8 might evolve from the identification of Nek9 phosphosites described above (Figure 30). By systematically generating appropriate phospho-deficient and phospho-mimicking Eps8 mutants and subjecting them to in vitro phosphorylation and ubiquitylation assays, one should be able to identify the Fbxw5 degron(s) within Eps8. Once known, these 
sequences could be used for crystallization of Fbxw5 with Eps8-degron peptides and for identification of more Fbxw5 target candidates by database searches. 


\section{Eps8 is a target of Fbxw5-dependent ubiquitylation - when, how, and why?}

Since the identification of Eps8 as a substrate for the epidermal growth factor receptor (EGFR) (Fazioli et al. 1993), biochemical, structural, cell biological, and genetic evidence has provided a detailed picture of its versatile functions. Eps8 has been shown to participate in the formation of several functionally distinct macromolecular complexes, which mediate actin cytoskeleton rearrangements (e.g. by transducing signals from Ras to Rac) or endocytosis of receptor tyrosine kinases via Rab5 (Figure 34). At a molecular level, Eps8 has been shown to regulate actin cytoskeleton dynamics at least by three different mechanisms: When in complex with Abi1, Eps8 exerts actin capping activity, thereby controlling the length of actin polymers (Croce et al. 2004; Disanza et al. 2004). A trimeric Eps8-Abi1-Sos1 complex can promote actin bundling via Rac signaling (Scita et al. 1999; Scita et al. 2001), and complexes formed by Eps8 and IRSp53 can directly mediate actin crosslinking in a Cdc42-regulated manner (Disanza et al. 2006). The property of Eps8 to exert actin capping and crosslinking activity at the same time is based on the ability of its C-terminal effector region to bind actin structures via two different binding modes. For actin capping, Eps8 binds to the barbed-ends of actin polymers. For localizing complexes that signal or mediate actin bundling Eps8 can bind to actin filament sides (Hertzog et al. 2010).

Hence, Eps8 is a bifunctional actin remodeler that controls various actin-based protrusion in cells, thereby acting as a positive regulator of cell proliferation and motility. As expected from these functions, deregulation of Eps8 levels and activity has been shown to play a key role in pathological contexts, such as during tumor development. Eps8 is upregulated in a variety of tumor types and has been reported to be specifically required for optimal cell migration and invasion in a subset of metastatic oral squamous carcinoma cells (Wang et al. 2009; Yap et al. 2009). Despite these observations, little is known about how the Eps8 protein levels are controlled and how Eps8 recruitment into functionally distinct complexes is regulated within cells. There is only one very recent report, which provides evidence for a first degradation mechanism of Eps8 independent of ubiqutiylation (Welsch et al. 2010). When expressed at high levels (e.g. such as 
in a number of pancreatic cancer cell lines), Eps8 is subject to chaperonemediated autophagy under normal cellular growth conditions.

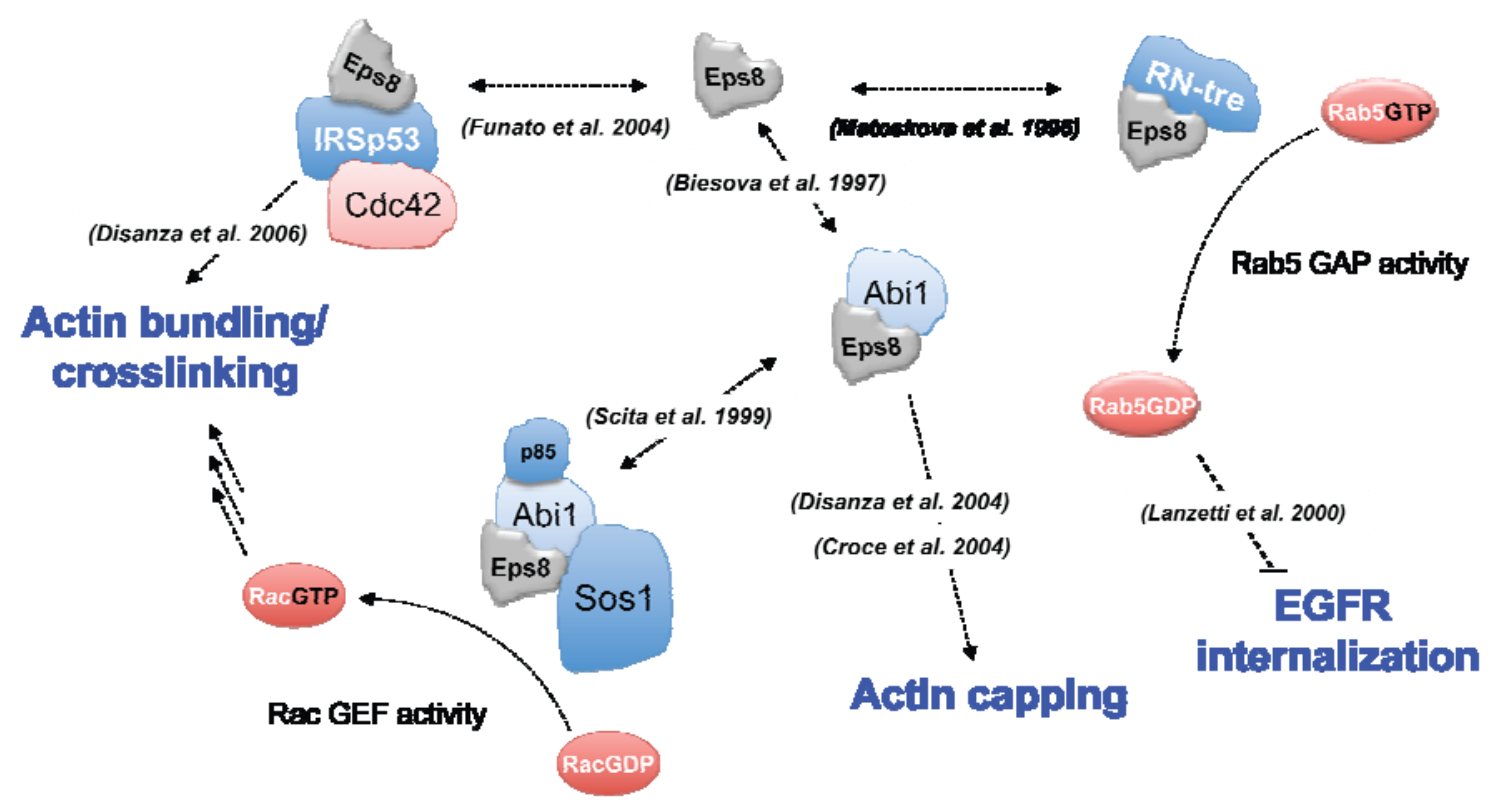

Figure 34: Eps8 regulates actin cytoskeleton remodeling and receptor endocytosis. Via its SH3 domain, Eps8 can associate with RN-tre (Matoskova et al. 1996). By entering in a complex with RN-tre, Eps8 promotes the GAP activity of RN-tre towards Rab5, thereby inhibiting EGFR internalization (Lanzetti et al. 2000). Alternatively, Abi1 can bind to the SH3 domain of Eps8 (Biesova, Piccoli, and Wong 1997), thereby releasing the auto-inhibited capping activity of Eps8 (Croce et al. 2004; Disanza et al. 2004). The Eps8-Abi1 complex can further associate with Sos1 and the regulatory subunit of the PI3K, p85, to form a complex that exhibits Rac-specific GEF activity (Scita et al. 1999), which ultimately contributes to membrane ruffling (Scita et al. 2001). Furthermore, via a multi-surface interaction, Eps8 can associate with IRSp53 (Funato et al. 2004). The Eps8-IRsp53 complex has been demonstrated to have a Cdc42-regulated actin bundling activity important for formation of filipodial protrusions (Disanza et al. 2006). Recently, the molecular basis for the dual function of Eps8 on actin dynamics (bundling and capping) has been elucidated (Hertzog et al. 2010).

\subsection{Possible functions of Eps8 ubiquitylation by $\mathrm{SCF}^{\mathrm{Fbxw5}}$}

Within this work, I have provided compelling biochemical evidence that Eps8 is a substrate of $\mathrm{SCF}^{\mathrm{Fbxw5}}$-mediated ubiquitylation (Figure 18, page 90; Figure 20, page 92). In addition, endogenous Eps8 co-purifies with Fbxw5 (Figure 14, page 83) and Fbxw5 downregulation leads to an increase in Eps8 levels in HeLa cells (Figure 17, page 88), clearly suggesting that Fbxw5-mediated Eps8 ubiquitylation regulates Eps8 levels in vivo. Yet, since the increase of Eps8 levels upon Fbxw5 
knock down was rather moderate and Eps8 seems to be a stable protein under normal cellular growth conditions, there is also the possibility that Fbxw5mediated Eps8 ubiquitylation serves purposes different from degradation. It is well documented that Lys48-linked polyubiquitination can activate an Ubiquitinselective chaperone termed p97 (Jentsch and Rumpf 2007). p97 is a member of the AAA (ATPase associated with various cellular activities) ATPase family. It can cooperate with distinctive cofactors to differentially act on various substrates conjugated with Lys48-linked polyubiquitin chains and segregate ubiquitylated substrates from unmodified binding partners. While this leads to proteasomemediated degradation in the ERAD pathway, there is emerging evidence that such a "segregase" function of p97 is also required in different cellular contexts. For example, Meyer and colleagues demonstrated that p97 in complex with its cofactor complex Ufd1-Npl4 can act on polyubiquitiylated Aurora B kinase to extract it from chromatin during mitosis, which allows chromosome decondensation and the reformation of the nuclear envelope (Ramadan et al. 2007). In light of the fact that Eps8 is known to assemble in a variety of functionally distinct complexes, it is intriguing to speculate that $\mathrm{SCF}^{\mathrm{Fbxw5}}$-mediated ubiquitylation might serve as a trigger to activate Eps8 complex remodeling by a similar mechanism. In such a scenario, some Eps8 molecules might be captured and degraded by the proteasome, however the main purpose of ubiquitylation would be to orchestrate the molecular interactions of Eps8.

As experimental data for a role of ubiquitylation in Eps8 complex remodeling is currently still missing, I will focus the following discussion on putative functions in degradation.

\subsection{Fbxw5-mediated Eps8 degradation - stimulus- or sub-pool- dependent?}

Within this study, Eps8 purified from insect or mammalian cells could be efficiently ubiquitylated by either immunoprecipitated or in vitro reconstituted $\mathrm{SCF}^{\mathrm{Fbxw} 5}$ complexes. In addition, downregulation of Fbxw5 in HeLa cells resulted in an accumulation of Eps8. These data clearly suggested that ubiquitylation by Fbxw5 triggers the degradation of Eps8 in vivo. Yet, it was neither possible to 
detect a decrease in Eps8 levels nor to identify ubiquitylated species of endogenous or exogenous Eps8 in the presence of overexpressed Fbxw5 (result chapter 3.1, page 85 and data not shown). What are the factors that might render the isolation of ubiquitylated Eps8 species from cells so difficult?

From the in vitro ubiquitylation assays, we can deduce the following conclusions: Overexpressed Fbxw5 is integrated into functional SCF complexes in vivo and the majority of the Eps8 pool can be recognized and ubiquitylated by Fbxw5 when purified from cells. Thus, both components are by themselves in principle primed for ubiquitylation. Furthermore, the in vitro system is sufficient for Eps8 ubiquitylation, rendering the involvement of co-factors such as in the case for Fbxl1 (Hao et al. 2005) rather unlikely. These findings make one even further wonder, why simple overexpression of Fbxw5, which clearly results in more SCF $^{\text {Fbxw5 }}$ complex (Figure 9, page 74), does not result in detectable Eps8 ubiquitylation and degradation in vivo. Possible answers for this question might be provided by the observation that Eps8 accumulation after downregulation of Fbxw5 was most profound, when cells were treated according to a precisely scheduled $72 \mathrm{~h}$ culturing protocol (Figure 17, page 88). This observation suggests that Eps8 degradation requires a certain stimulus. Moreover, only a sub-pool of cellular Eps8 may be targeted by Fbxw5 under normal cellular growth conditions - too small to be detected via immunoblotting. In addition, specific isopeptidases that could readily reverse Eps8 ubiquitylation might further complicate the detection of cellular, Fbxw5-dependent Eps8-Ubiquitin conjugates.

In summary, these observations suggest that Fbxw5 targets either only a subpool of Eps8 or requires a specific stimulus for efficient Eps8 ubiquitylation and degradation in vivo, rather than controlling general Eps8 levels under normal cellular growth conditions.

\subsection{Nek9 - a putative regulator of Eps8 degradation?}

Originally identified as a kinase essential for proper mitotic spindle assembly (reviewed in (O'Regan, Blot, and Fry 2007)), the NIMA-related kinase 9 (Nek9) has recently been shown to regulate autophagy (Behrends et al. 2010). In a mass spectrometry-based screen to characterize the autophagy interaction network in 
human cells under conditions of basal autophagy, Nek9 was identified to interact with all six human LC3/ATG8 homologues. Importantly, downregulation of Nek9 with four different siRNAs resulted in a decrease in autophagosome number under basal and rapamycin-induced autophagy conditions. While these findings clearly demonstrated that Nek9 regulates autophagy, they do not to clarify by which mechanism. The number of autophagosomes at a given time point is a function of the balance between the rate of their generation and the rate of their conversion into autophagolysosomes (Mizushima, Yoshimori, and Levine 2010). Hence, Nek9 could either be a positive regulator of autophagosome formation, or an inhibitor of the conversion of autophagosomes into autophagolysosomes.

Within this study, I have shown that overexpression of a constitutively active Nek9 version stabilizes Eps8 levels in HeLa cells (Figure 29, page 103). Assuming a negative role of Nek9 in autophagy, this observation could be explained in the following model. Under normal growth conditions, Eps8 is slowly degraded via an autophagy mechanism and would accumulate in cells upon general inhibition of autophagy via overexpression of Nek9. Yet, in light of the fact that under normal growth conditions Eps8 endogenously co-purifies with Nek9 (Figure 24, page 97) and Nek9 efficiently phosphorylates Eps8 in vitro (Figure 25, page 98), such an indirect scenario seems rather unlikely.

Simultaneously to this study, Welsch and colleagues have provided that Eps8 can be a target of chaperone-mediated autophagy under normal cellular growth conditions (Welsch et al. 2010). Since such a mechanism involves targeting of Eps8 to lysosomes via Hsc70 and accessory chaperones, it would be conceivable that phosphorylation of Eps8 by Nek9 could interfere with this targeting mechanism thereby stabilizing Eps8.

A third possibility to explain the increase of Eps8 levels upon Nek9 overexpression evolves from the results of combined in vitro kinase and ubiquitylation assays, which suggest that Nek9 acts as a negative regulator of $\mathrm{SCF}^{\mathrm{Fbxw}}$-dependent ubiquitylation by phosphorylating Eps8 (Figure 27, page 100). Hence, Nek9 could block the Fbxw5-mediated ubiquitylation of Eps8 also in vivo and thereby prevent its degradation. Whether such an Ubiquitin-dependent degradation mechanism would dependent on proteasomes or lysosomes is currently unclear. My own preliminary data suggest that Eps8 can be degraded 
via lysosomes in HeLa cells upon serum starvation (data not shown). Since there is a recently discovered Ubiquitin-dependent autophagy pathway (see introduction section 3.1.2, page 16), this observation in principle offers the possibility that Fbxw5-mediated Eps8 degradation could occur via a selelctive autophagy mechanism.

Taken together, I here presented the most straight-forward models for Eps8 regulation by Nek9 in cells. Yet, since it is currently unknown how Nek9 regulates autophagy and how Eps8 ubiquitylation leads to degradation, many more scenarios are of course conceivable. So what are possible approaches to clarify these issues? First, the precise role of Nek9 in autophagy could be determined by investigating the changes of the autophagic flux upon depletion of Nek9 from cells e.g. via LC3 turnover assays, measurement of p62 levels, or via different fluorescence-based autophagy assays (Mizushima, Yoshimori, and Levine 2010). These types of experiments could also be performed in the presence of overexpressed, constitutively active, or catalytically dead Nek9 mutants to characterize the role of Nek9's kinase activity in autophagy regulation. Once this role is established, one would further include the analysis of the phosphorylation status, levels, and localization of Eps8 and Fbxw5 upon downregulation and overexpression of Nek9 under various autophagy conditions.

Taking these approaches, it will be interesting to elucidate by which mechanism(s) Nek9 regulates Eps8 degradation in cells.

\subsection{Identification of Eps8 as a target for Fbxw5 - general roles for Fbxw5 in cell growth or migration?}

Within this work, I have provided evidence that Fbxw5 is a regulator involved in Eps8 stability on the protein level. Given that Eps8 levels are elevated in various types of cancer including pancreatic (Welsch et al. 2007), colon (Maa et al. 2007), and oral (Yap et al. 2009) cancer, it is intriguing to speculate that at least in some cases, this increase is due to disturbances in Fbxw5-mediated Eps8 degradation. Future studies investigating this hypothesis might include the analysis of Fbxw5 levels in appropriate cancer cells and the test for an inverse correlation with Eps8 levels. Alternatively, the impact of Fbxw5 downregulation or overexpression on 
eps8-mediated cell migration and invasion processes could be assayed in e.g. different oral squamous carcinoma cell lines.

F-box proteins are thought to simultaneously target many different substrates for degradation in order to fulfill a certain physiological purpose (Skaar et al. 2009). While it is of course not possible to deduce a general role from only one target, a first piece of evidence for a broader regulatory function of $\mathrm{SCF}^{\mathrm{Fbxw} 5}$ in cell migration might be provided by the identification of the myristoylated alanine-rich C-kinase substrate MARCKS as a putative Fbxw5 interaction partner (Table 2, page 82). MARCKS - similar to Eps8 - is an actin filament crosslinking molecule (Hartwig et al. 1992), that has been implicated in cell adhesion, spreading and motility (Arbuzova, Schmitz, and Vergeres 2002). It will be interesting to see, whether MARCKS is indeed an interactor and substrate of Fbxw5.

\section{Concluding remarks and perspectives}

While the results presented in this work are far from providing a complete understanding of the mechanistic and functional properties of the F-box protein Fbxw5, they substantially extend our knowledge about Fbxw5 in four ways:

1) Fbxw5 endogenously associates to components of SCF and CRL4 complexes

2) Eps8 is the first target of Fbxw5 in context of SCF complexes

3) Fbxw5 is a regulator of Eps8 protein levels, suggesting putative roles of Fbxw5 in cellular motility and cancer development

4) $\mathrm{SCF}^{\mathrm{Fbxw5}}$-mediated Eps8 ubiquitylation is negatively regulated by Nek9 phosphorylation, suggesting a phosphorylation-inhibited Fbxw5 degron within Eps8

Moreover, overexpression of Nek9 stabilizes Eps8 levels in cells. While experimental proof is still missing, this raises the possibility that Nek9 might also inhibit SCF ${ }^{\mathrm{Fbxw5}}$-mediated Eps8 ubiquitylation and degradation in vivo.

Intriguing questions worth pursuing in future studies include e.g. in which way Fbxw5's ability to assemble into two distinct CRL complexes impacts on its 
activity or substrate specificity and what the functional interplay of Fbxw5, Eps8, and Nek9 looks like in vivo. 


\section{REFERENCES}

Arbuzova, A., A. A. Schmitz, and G. Vergeres. 2002. Cross-talk unfolded: MARCKS proteins. Biochem J 362:1-12.

Azzalin, C. M., and J. Lingner. 2006. The human RNA surveillance factor UPF1 is required for $S$ phase progression and genome stability. Curr Biol 16:433439.

Behrends, C., M. E. Sowa, S. P. Gygi, and J. W. Harper. 2010. Network organization of the human autophagy system. Nature 466:68-76.

Belham, C., J. Roig, J. A. Caldwell, Y. Aoyama, B. E. Kemp, M. Comb, and J. Avruch. 2003. A mitotic cascade of NIMA family kinases. Nercc1/Nek9 activates the Nek6 and Nek7 kinases. J Biol Chem 278:34897-34909.

Biesova, Z., C. Piccoli, and W. T. Wong. 1997. Isolation and characterization of e3B1, an eps8 binding protein that regulates cell growth. Oncogene 14:233-241.

Birnboim, H. C., and J. Doly. 1979. A rapid alkaline extraction procedure for screening recombinant plasmid DNA. Nucleic Acids Res 7:1513-1523.

Bradford, M. M. 1976. A rapid and sensitive method for the quantitation of microgram quantities of protein utilizing the principle of protein-dye binding. Anal Biochem 72:248-254.

Butz, N., S. Ruetz, F. Natt, J. Hall, J. Weiler, J. Mestan, M. Ducarre, R. Grossenbacher, P. Hauser, D. Kempf, and F. Hofmann. 2005. The human ubiquitin-conjugating enzyme $\mathrm{Cdc} 34$ controls cellular proliferation through regulation of p27Kip1 protein levels. Exp Cell Res 303:482-493.

Cardozo, T., and M. Pagano. 2004. The SCF ubiquitin ligase: insights into a molecular machine. Nat Rev Mol Cell Biol 5:739-751.

Ciechanover, A., H. Heller, S. Elias, A. L. Haas, and A. Hershko. 1980. ATPdependent conjugation of reticulocyte proteins with the polypeptide required for protein degradation. Proc Natl Acad Sci U S A 77:1365-1368.

Conaway, R. C., C. S. Brower, and J. W. Conaway. 2002. Emerging roles of ubiquitin in transcription regulation. Science 296:1254-1258. 
Cope, G. A., and R. J. Deshaies. 2006. Targeted silencing of Jab1/Csn5 in human cells downregulates SCF activity through reduction of F-box protein levels. BMC Biochem 7:1.

Cope, G. A., and R. J. Deshaies. 2003. COP9 signalosome: a multifunctional regulator of SCF and other cullin-based ubiquitin ligases. Cell 114:663671.

Cope, G. A., G. S. Suh, L. Aravind, S. E. Schwarz, S. L. Zipursky, E. V. Koonin, and R. J. Deshaies. 2002. Role of predicted metalloprotease motif of Jab1/Csn5 in cleavage of Nedd8 from Cul1. Science 298:608-611.

Croce, A., G. Cassata, A. Disanza, M. C. Gagliani, C. Tacchetti, M. G. Malabarba, M. F. Carlier, G. Scita, R. Baumeister, and P. P. Di Fiore. 2004. A novel actin barbed-end-capping activity in EPS-8 regulates apical morphogenesis in intestinal cells of Caenorhabditis elegans. Nat Cell Biol 6:1173-1179.

Deshaies, R. 2005. Methods in Enzymology: Ubiquitin and Protein Degradation, Part A/B, Volumes 389/399. Elsevier Academic Press.

Deshaies, R. J., and C. A. Joazeiro. 2009. RING domain E3 ubiquitin ligases. Annu Rev Biochem 78:399-434.

Di Fiore, P. P., and G. Scita. 2002. Eps8 in the midst of GTPases. Int J Biochem Cell Biol 34:1178-1183.

Dias, D. C., G. Dolios, R. Wang, and Z. Q. Pan. 2002. CUL7: A DOC domaincontaining cullin selectively binds Skp1.Fbx29 to form an SCF-like complex. Proc Natl Acad Sci U S A 99:16601-16606.

Dikic, I., S. Wakatsuki, and K. J. Walters. 2009. Ubiquitin-binding domains - from structures to functions. Nat Rev Mol Cell Biol 10:659-671.

Disanza, A., M. F. Carlier, T. E. Stradal, D. Didry, E. Frittoli, S. Confalonieri, A. Croce, J. Wehland, P. P. Di Fiore, and G. Scita. 2004. Eps8 controls actinbased motility by capping the barbed ends of actin filaments. Nat Cell Biol 6:1180-1188.

Disanza, A., S. Mantoani, M. Hertzog, S. Gerboth, E. Frittoli, A. Steffen, K. Berhoerster, H. J. Kreienkamp, F. Milanesi, P. P. Di Fiore, A. Ciliberto, T. E. Stradal, and G. Scita. 2006. Regulation of cell shape by Cdc42 is 
mediated by the synergic actin-bundling activity of the Eps8-IRSp53 complex. Nat Cell Biol 8:1337-1347.

Dorrello, N. V., A. Peschiaroli, D. Guardavaccaro, N. H. Colburn, N. E. Sherman, and M. Pagano. 2006. S6K1- and betaTRCP-mediated degradation of PDCD4 promotes protein translation and cell growth. Science 314:467471.

Duda, D. M., L. A. Borg, D. C. Scott, H. W. Hunt, M. Hammel, and B. A. Schulman. 2008. Structural insights into NEDD8 activation of cullin-RING ligases: conformational control of conjugation. Cell 134:995-1006.

Elbashir, S. M., J. Harborth, W. Lendeckel, A. Yalcin, K. Weber, and T. Tuschl. 2001. Duplexes of 21-nucleotide RNAs mediate RNA interference in cultured mammalian cells. Nature 411:494-498.

Fazioli, F., L. Minichiello, V. Matoska, P. Castagnino, T. Miki, W. T. Wong, and P. P. Di Fiore. 1993. Eps8, a substrate for the epidermal growth factor receptor kinase, enhances EGF-dependent mitogenic signals. EMBO J 12:3799-3808.

Finley, D. 2009. Recognition and processing of ubiquitin-protein conjugates by the proteasome. Annu Rev Biochem 78:477-513.

Frescas, D., and M. Pagano. 2008. Deregulated proteolysis by the F-box proteins SKP2 and beta-TrCP: tipping the scales of cancer. Nat Rev Cancer 8:438449.

Funato, Y., T. Terabayashi, N. Suenaga, M. Seiki, T. Takenawa, and H. Miki. 2004. IRSp53/Eps8 complex is important for positive regulation of Rac and cancer cell motility/invasiveness. Cancer Res 64:5237-5244.

Galan, J. M., and M. Peter. 1999. Ubiquitin-dependent degradation of multiple Fbox proteins by an autocatalytic mechanism. Proc Natl Acad Sci U S A 96:9124-9129.

Gauci, S., A. O. Helbig, M. Slijper, J. Krijgsveld, A. J. Heck, and S. Mohammed. 2009. Lys-N and trypsin cover complementary parts of the phosphoproteome in a refined SCX-based approach. Anal Chem 81:44934501.

Glickman, M. H., and A. Ciechanover. 2002. The ubiquitin-proteasome proteolytic pathway: destruction for the sake of construction. Physiol Rev 82:373-428. 
Graham, F. L., and A. J. van der Eb. 1973. A new technique for the assay of infectivity of human adenovirus 5 DNA. Virology 52:456-467.

Groettrup, M., C. Pelzer, G. Schmidtke, and K. Hofmann. 2008. Activating the ubiquitin family: UBA6 challenges the field. Trends Biochem Sci 33:230237.

Haglund, K., S. Sigismund, S. Polo, I. Szymkiewicz, P. P. Di Fiore, and I. Dikic. 2003. Multiple monoubiquitination of RTKs is sufficient for their endocytosis and degradation. Nat Cell Biol 5:461-466.

Hao, B., S. Oehlmann, M. E. Sowa, J. W. Harper, and N. P. Pavletich. 2007. Structure of a Fbw7-Skp1-cyclin E complex: multisite-phosphorylated substrate recognition by SCF ubiquitin ligases. Mol Cell 26:131-143.

Hao, B., N. Zheng, B. A. Schulman, G. Wu, J. J. Miller, M. Pagano, and N. P. Pavletich. 2005. Structural basis of the Cks1-dependent recognition of p27(Kip1) by the SCF(Skp2) ubiquitin ligase. Mol Cell 20:9-19.

Hartwig, J. H., M. Thelen, A. Rosen, P. A. Janmey, A. C. Nairn, and A. Aderem. 1992. MARCKS is an actin filament crosslinking protein regulated by protein kinase $C$ and calcium-calmodulin. Nature 356:618-622.

Hashizume, R., M. Fukuda, I. Maeda, H. Nishikawa, D. Oyake, Y. Yabuki, H. Ogata, and T. Ohta. 2001. The RING heterodimer BRCA1-BARD1 is a ubiquitin ligase inactivated by a breast cancer-derived mutation. J Biol Chem 276:14537-14540.

He, C., and D. J. Klionsky. 2009. Regulation mechanisms and signaling pathways of autophagy. Annu Rev Genet 43:67-93.

He, Y. J., C. M. McCall, J. Hu, Y. Zeng, and Y. Xiong. 2006. DDB1 functions as a linker to recruit receptor WD40 proteins to CUL4-ROC1 ubiquitin ligases. Genes Dev 20:2949-2954.

Hermand, D. 2006. F-box proteins: more than baits for the SCF? Cell Div 1:30. Hershko, A., and A. Ciechanover. 1998. The ubiquitin system. Annu Rev Biochem 67:425-479.

Hertzog, M., F. Milanesi, L. Hazelwood, A. Disanza, H. Liu, E. Perlade, M. G. Malabarba, S. Pasqualato, A. Maiolica, S. Confalonieri, C. Le Clainche, N. Offenhauser, J. Block, K. Rottner, P. P. Di Fiore, M. F. Carlier, N. Volkmann, D. Hanein, and G. Scita. 2010. Molecular basis for the dual 
function of Eps8 on actin dynamics: bundling and capping. PLoS Biol 8:e1000387.

Hicke, L., and R. Dunn. 2003. Regulation of membrane protein transport by ubiquitin and ubiquitin-binding proteins. Annu Rev Cell Dev Biol 19:141172.

Hochstrasser, M. 2009. Origin and function of ubiquitin-like proteins. Nature 458:422-429.

Holland, P. M., A. Milne, K. Garka, R. S. Johnson, C. Willis, J. E. Sims, C. T. Rauch, T. A. Bird, and G. D. Virca. 2002. Purification, cloning, and characterization of Nek8, a novel NIMA-related kinase, and its candidate substrate Bicd2. J Biol Chem 277:16229-16240.

Hori, T., F. Osaka, T. Chiba, C. Miyamoto, K. Okabayashi, N. Shimbara, S. Kato, and K. Tanaka. 1999. Covalent modification of all members of human cullin family proteins by NEDD8. Oncogene 18:6829-6834.

Hu, J., S. Zacharek, Y. J. He, H. Lee, S. Shumway, R. J. Duronio, and Y. Xiong. 2008. WD40 protein FBW5 promotes ubiquitination of tumor suppressor TSC2 by DDB1-CUL4-ROC1 ligase. Genes Dev 22:866-871.

Hurley, J. H., S. Lee, and G. Prag. 2006. Ubiquitin-binding domains. Biochem J 399:361-372.

Imai, Y., M. Soda, and R. Takahashi. 2000. Parkin suppresses unfolded protein stress-induced cell death through its E3 ubiquitin-protein ligase activity. J Biol Chem 275:35661-35664.

Jentsch, S., and S. Rumpf. 2007. Cdc48 (p97): a "molecular gearbox" in the ubiquitin pathway? Trends Biochem Sci 32:6-11.

Jin, J., T. Cardozo, R. C. Lovering, S. J. Elledge, M. Pagano, and J. W. Harper. 2004. Systematic analysis and nomenclature of mammalian F-box proteins. Genes Dev 18:2573-2580.

Jin, L., A. Williamson, S. Banerjee, I. Philipp, and M. Rape. 2008. Mechanism of ubiquitin-chain formation by the human anaphase-promoting complex. Cell 133:653-665.

Kirkin, V., D. G. McEwan, I. Novak, and I. Dikic. 2009. A role for ubiquitin in selective autophagy. Mol Cell 34:259-269. 
Kirkpatrick, D. S., N. A. Hathaway, J. Hanna, S. Elsasser, J. Rush, D. Finley, R. W. King, and S. P. Gygi. 2006. Quantitative analysis of in vitro ubiquitinated cyclin B1 reveals complex chain topology. Nat Cell Biol 8:700-710.

Komander, D. 2009. The emerging complexity of protein ubiquitination. Biochem Soc Trans 37:937-953.

Komander, D., M. J. Clague, and S. Urbe. 2009. Breaking the chains: structure and function of the deubiquitinases. Nat Rev Mol Cell Biol 10:550-563.

Korolchuk, V. I., F. M. Menzies, and D. C. Rubinsztein. 2010. Mechanisms of cross-talk between the ubiquitin-proteasome and autophagy-lysosome systems. FEBS Lett 584:1393-1398.

Kroemer, G., and B. Levine. 2008. Autophagic cell death: the story of a misnomer. Nat Rev Mol Cell Biol 9:1004-1010.

Kyhse-Andersen, J. 1984. Electroblotting of multiple gels: a simple apparatus without buffer tank for rapid transfer of proteins from polyacrylamide to nitrocellulose. J Biochem Biophys Methods 10:203-209.

Laemmli, U. K. 1970. Cleavage of structural proteins during the assembly of the head of bacteriophage T4. Nature 227:680-685.

Lanzetti, L., V. Rybin, M. G. Malabarba, S. Christoforidis, G. Scita, M. Zerial, and P. P. Di Fiore. 2000. The Eps8 protein coordinates EGF receptor signalling through Rac and trafficking through Rab5. Nature 408:374-377.

Li, T., N. P. Pavletich, B. A. Schulman, and N. Zheng. 2005. High-level expression and purification of recombinant SCF ubiquitin ligases. Methods Enzymol 398:125-142.

Liu, W., A. F. Nichols, J. A. Graham, R. Dualan, A. Abbas, and S. Linn. 2000. Nuclear transport of human DDB protein induced by ultraviolet light. J Biol Chem 275:21429-21434.

Luckow, V. A., S. C. Lee, G. F. Barry, and P. O. Olins. 1993. Efficient generation of infectious recombinant baculoviruses by site-specific transposonmediated insertion of foreign genes into a baculovirus genome propagated in Escherichia coli. J Virol 67:4566-4579.

Maa, M. C., J. C. Lee, Y. J. Chen, Y. C. Lee, S. T. Wang, C. C. Huang, N. H. Chow, and T. H. Leu. 2007. Eps8 facilitates cellular growth and motility of 
colon cancer cells by increasing the expression and activity of focal adhesion kinase. J Biol Chem 282:19399-19409.

Mahajan, R., C. Delphin, T. Guan, L. Gerace, and F. Melchior. 1997. A small ubiquitin-related polypeptide involved in targeting RanGAP1 to nuclear pore complex protein RanBP2. Cell 88:97-107.

Markson, G., C. Kiel, R. Hyde, S. Brown, P. Charalabous, A. Bremm, J. Semple, J. Woodsmith, S. Duley, K. Salehi-Ashtiani, M. Vidal, D. Komander, L. Serrano, P. Lehner, and C. M. Sanderson. 2009. Analysis of the human E2 ubiquitin conjugating enzyme protein interaction network. Genome Res 19:1905-1911.

Matoskova, B., W. T. Wong, N. Nomura, K. C. Robbins, and P. P. Di Fiore. 1996. $\mathrm{RN}$-tre specifically binds to the SH3 domain of eps8 with high affinity and confers growth advantage to NIH3T3 upon carboxy-terminal truncation. Oncogene 12:2679-2688.

Matoskova, B., W. T. Wong, A. E. Salcini, P. G. Pelicci, and P. P. Di Fiore. 1995. Constitutive phosphorylation of eps8 in tumor cell lines: relevance to malignant transformation. Mol Cell Biol 15:3805-3812.

Matyskiela, M. E., M. C. Rodrigo-Brenni, and D. O. Morgan. 2009. Mechanisms of ubiquitin transfer by the anaphase-promoting complex. J Biol 8:92.

Menna, E., A. Disanza, C. Cagnoli, U. Schenk, G. Gelsomino, E. Frittoli, M. Hertzog, N. Offenhauser, C. Sawallisch, H. J. Kreienkamp, F. B. Gertler, P. P. Di Fiore, G. Scita, and M. Matteoli. 2009. Eps8 regulates axonal filopodia in hippocampal neurons in response to brain-derived neurotrophic factor (BDNF). PLoS Biol 7:e1000138.

Merlet, J., J. Burger, J. E. Gomes, and L. Pintard. 2009. Regulation of cullin-RING E3 ubiquitin-ligases by neddylation and dimerization. Cell Mol Life Sci 66:1924-1938.

Meulmeester, E., M. Kunze, H. H. Hsiao, H. Urlaub, and F. Melchior. 2008. Mechanism and consequences for paralog-specific sumoylation of ubiquitin-specific protease 25. Mol Cell 30:610-619.

Minoda, Y., H. Sakurai, T. Kobayashi, A. Yoshimura, and G. Takaesu. 2009. An F-box protein, FBXW5, negatively regulates TAK1 MAP3K in the IL-1beta signaling pathway. Biochem Biophys Res Commun 381:412-417. 
Mizushima, N., B. Levine, A. M. Cuervo, and D. J. Klionsky. 2008. Autophagy fights disease through cellular self-digestion. Nature 451:1069-1075.

Mizushima, N., T. Yoshimori, and B. Levine. 2010. Methods in mammalian autophagy research. Cell 140:313-326.

Moldovan, G. L., B. Pfander, and S. Jentsch. 2007. PCNA, the maestro of the replication fork. Cell 129:665-679.

Mullis, K., F. Faloona, S. Scharf, R. Saiki, G. Horn, and H. Erlich. 1986. Specific enzymatic amplification of DNA in vitro: the polymerase chain reaction. Cold Spring Harb Symp Quant Biol 51 Pt 1:263-273.

Neuhoff, V., Stamm, R., Eibl, H. 1985. Clear background and highly sensitive protein staining with Coomassie Blue dyes in polyacrylamide gels: A systematic analysis. Electrophoresis 6, 427-448.

O'Regan, L., J. Blot, and A. M. Fry. 2007. Mitotic regulation by NIMA-related kinases. Cell Div 2:25.

Olsen, J. V., B. Blagoev, F. Gnad, B. Macek, C. Kumar, P. Mortensen, and M. Mann. 2006. Global, in vivo, and site-specific phosphorylation dynamics in signaling networks. Cell 127:635-648.

Osaka, F., M. Saeki, S. Katayama, N. Aida, E. A. Toh, K. Kominami, T. Toda, T. Suzuki, T. Chiba, K. Tanaka, and S. Kato. 2000. Covalent modifier NEDD8 is essential for SCF ubiquitin-ligase in fission yeast. EMBO J 19:34753484 .

Paoli, M. 2001. Protein folds propelled by diversity. Prog Biophys Mol Biol 76:103130.

Petroski, M. D., and R. J. Deshaies. 2005. Function and regulation of cullin-RING ubiquitin ligases. Nat Rev Mol Cell Biol 6:9-20.

Pichler, A., A. Gast, J. S. Seeler, A. Dejean, and F. Melchior. 2002. The nucleoporin RanBP2 has SUMO1 E3 ligase activity. Cell 108:109-120.

Pickart, C. M. 2001. Mechanisms underlying ubiquitination. Annu Rev Biochem 70:503-533.

Pickart, C. M. 2000. Ubiquitin in chains. Trends Biochem Sci 25:544-548.

Pickart, C. M., and M. J. Eddins. 2004. Ubiquitin: structures, functions, mechanisms. Biochim Biophys Acta 1695:55-72. 
Pickart, C. M., and S. Raasi. 2005. Controlled synthesis of polyubiquitin chains. Methods Enzymol 399:21-36.

Pintard, L., A. Willems, and M. Peter. 2004. Cullin-based ubiquitin ligases: Cul3BTB complexes join the family. EMBO J 23:1681-1687.

Rabut, G., and M. Peter. 2008. Function and regulation of protein neddylation. 'Protein modifications: beyond the usual suspects' review series. EMBO Rep 9:969-976.

Ramadan, K., R. Bruderer, F. M. Spiga, O. Popp, T. Baur, M. Gotta, and H. H. Meyer. 2007. Cdc48/p97 promotes reformation of the nucleus by extracting the kinase Aurora B from chromatin. Nature 450:1258-1262.

Rikova, K., A. Guo, Q. Zeng, A. Possemato, J. Yu, H. Haack, J. Nardone, K. Lee, C. Reeves, Y. Li, Y. Hu, Z. Tan, M. Stokes, L. Sullivan, J. Mitchell, R. Wetzel, J. Macneill, J. M. Ren, J. Yuan, C. E. Bakalarski, J. Villen, J. M. Kornhauser, B. Smith, D. Li, X. Zhou, S. P. Gygi, T. L. Gu, R. D. Polakiewicz, J. Rush, and M. J. Comb. 2007. Global survey of phosphotyrosine signaling identifies oncogenic kinases in lung cancer. Cell 131:1190-1203.

Rotin, D., and S. Kumar. 2009. Physiological functions of the HECT family of ubiquitin ligases. Nat Rev Mol Cell Biol 10:398-409.

Saeki, Y., T. Kudo, T. Sone, Y. Kikuchi, H. Yokosawa, A. Toh-e, and K. Tanaka. 2009. Lysine 63-linked polyubiquitin chain may serve as a targeting signal for the 26S proteasome. EMBO J 28:359-371.

Saha, A., and R. J. Deshaies. 2008. Multimodal activation of the ubiquitin ligase SCF by Nedd8 conjugation. Mol Cell 32:21-31.

Sanger, F., S. Nicklen, and A. R. Coulson. 1977. DNA sequencing with chainterminating inhibitors. Proc Natl Acad Sci U S A 74:5463-5467.

Schmidt, M. W., P. R. McQuary, S. Wee, K. Hofmann, and D. A. Wolf. 2009. Fbox-directed CRL complex assembly and regulation by the CSN and CAND1. Mol Cell 35:586-597.

Scita, G., J. Nordstrom, R. Carbone, P. Tenca, G. Giardina, S. Gutkind, M. Bjarnegard, C. Betsholtz, and P. P. Di Fiore. 1999. EPS8 and E3B1 transduce signals from Ras to Rac. Nature 401:290-293. 
Scita, G., P. Tenca, L. B. Areces, A. Tocchetti, E. Frittoli, G. Giardina, I. Ponzanelli, P. Sini, M. Innocenti, and P. P. Di Fiore. 2001. An effector region in Eps8 is responsible for the activation of the Rac-specific GEF activity of Sos-1 and for the proper localization of the Rac-based actinpolymerizing machine. J Cell Biol 154:1031-1044.

Shevchenko, A., M. Wilm, O. Vorm, and M. Mann. 1996. Mass spectrometric sequencing of proteins silver-stained polyacrylamide gels. Anal Chem 68:850-858.

Skaar, J. R., V. D'Angiolella, J. K. Pagan, and M. Pagano. 2009. SnapShot: F Box Proteins II. Cell 137:1358, 1358 e1351.

Skaug, B., X. Jiang, and Z. J. Chen. 2009. The role of ubiquitin in NF-kappaB regulatory pathways. Annu Rev Biochem 78:769-796.

Spence, J., R. R. Gali, G. Dittmar, F. Sherman, M. Karin, and D. Finley. 2000. Cell cycle-regulated modification of the ribosome by a variant multiubiquitin chain. Cell 102:67-76.

Strous, G. J., and R. Govers. 1999. The ubiquitin-proteasome system and endocytosis. J Cell Sci 112 ( Pt 10):1417-1423.

Sun, Z. W., and C. D. Allis. 2002. Ubiquitination of histone H2B regulates H3 methylation and gene silencing in yeast. Nature 418:104-108.

Tan, X., L. I. Calderon-Villalobos, M. Sharon, C. Zheng, C. V. Robinson, M. Estelle, and N. Zheng. 2007. Mechanism of auxin perception by the TIR1 ubiquitin ligase. Nature 446:640-645.

Tang, X., S. Orlicky, Z. Lin, A. Willems, D. Neculai, D. Ceccarelli, F. Mercurio, B. H. Shilton, F. Sicheri, and M. Tyers. 2007. Suprafacial orientation of the SCFCdc4 dimer accommodates multiple geometries for substrate ubiquitination. Cell 129:1165-1176.

Tokunaga, F., S. Sakata, Y. Saeki, Y. Satomi, T. Kirisako, K. Kamei, T. Nakagawa, M. Kato, S. Murata, S. Yamaoka, M. Yamamoto, S. Akira, T. Takao, K. Tanaka, and K. Iwai. 2009. Involvement of linear polyubiquitylation of NEMO in NF-kappaB activation. Nat Cell Biol 11:123132. 
Tsunematsu, R., M. Nishiyama, S. Kotoshiba, T. Saiga, T. Kamura, and K. I. Nakayama. 2006. Fbxw8 is essential for Cul1-Cul7 complex formation and for placental development. Mol Cell Biol 26:6157-6169.

Wang, H., V. Patel, H. Miyazaki, J. S. Gutkind, and W. A. Yeudall. 2009. Role for EPS8 in squamous carcinogenesis. Carcinogenesis 30:165-174.

Welchman, R. L., C. Gordon, and R. J. Mayer. 2005. Ubiquitin and ubiquitin-like proteins as multifunctional signals. Nat Rev Mol Cell Biol 6:599-609.

Welcker, M., and B. E. Clurman. 2008. FBW7 ubiquitin ligase: a tumour suppressor at the crossroads of cell division, growth and differentiation. Nat Rev Cancer 8:83-93.

Welsch, T., K. Endlich, T. Giese, M. W. Buchler, and J. Schmidt. 2007. Eps8 is increased in pancreatic cancer and required for dynamic actin-based cell protrusions and intercellular cytoskeletal organization. Cancer Lett 255:205-218.

Welsch, T., A. Younsi, A. Disanza, J. A. Rodriguez, A. M. Cuervo, G. Scita, and J. Schmidt. 2010. Eps8 is recruited to lysosomes and subjected to chaperone-mediated autophagy in cancer cells. Exp Cell Res 316:19141924.

Winston, J. T., D. M. Koepp, C. Zhu, S. J. Elledge, and J. W. Harper. 1999. A family of mammalian F-box proteins. Curr Biol 9:1180-1182.

Woelk, T., S. Sigismund, L. Penengo, and S. Polo. 2007. The ubiquitination code: a signalling problem. Cell Div 2:11.

Wollert, T., D. Yang, X. Ren, H. H. Lee, Y. J. Im, and J. H. Hurley. 2009. The ESCRT machinery at a glance. J Cell Sci 122:2163-2166.

Wu, G., G. Xu, B. A. Schulman, P. D. Jeffrey, J. W. Harper, and N. P. Pavletich. 2003. Structure of a beta-TrCP1-Skp1-beta-catenin complex: destruction motif binding and lysine specificity of the SCF(beta-TrCP1) ubiquitin ligase. Mol Cell 11:1445-1456.

Wu, K., J. Kovacev, and Z. Q. Pan. 2010. Priming and extending: a UbcH5/Cdc34 E2 handoff mechanism for polyubiquitination on a SCF substrate. Mol Cell 37:784-796.

Xu, P., D. M. Duong, N. T. Seyfried, D. Cheng, Y. Xie, J. Robert, J. Rush, M. Hochstrasser, D. Finley, and J. Peng. 2009. Quantitative proteomics 
reveals the function of unconventional ubiquitin chains in proteasomal degradation. Cell 137:133-145.

Xu, X., A. Sarikas, D. C. Dias-Santagata, G. Dolios, P. J. Lafontant, S. C. Tsai, W. Zhu, H. Nakajima, H. O. Nakajima, L. J. Field, R. Wang, and Z. Q. Pan. 2008. The CUL7 E3 ubiquitin ligase targets insulin receptor substrate 1 for ubiquitin-dependent degradation. Mol Cell 30:403-414.

Yang, C. S., C. Yu, H. C. Chuang, C. W. Chang, G. D. Chang, T. P. Yao, and H. Chen. 2005. FBW2 targets GCMa to the ubiquitin-proteasome degradation system. J Biol Chem 280:10083-10090.

Yap, L. F., V. Jenei, C. M. Robinson, K. Moutasim, T. M. Benn, S. P. Threadgold, V. Lopes, W. Wei, G. J. Thomas, and I. C. Paterson. 2009. Upregulation of Eps8 in oral squamous cell carcinoma promotes cell migration and invasion through integrin-dependent Rac1 activation. Oncogene 28:25242534.

Zheng, N., B. A. Schulman, L. Song, J. J. Miller, P. D. Jeffrey, P. Wang, C. Chu, D. M. Koepp, S. J. Elledge, M. Pagano, R. C. Conaway, J. W. Conaway, J. W. Harper, and N. P. Pavletich. 2002. Structure of the Cul1-Rbx1-Skp1-F boxSkp2 SCF ubiquitin ligase complex. Nature 416:703-709. 


\section{ABBREVIATIONS}

\section{General abbreviations}

aa

A

ADP

APS

ATP

BSA

C

C-

cDNA

CIP

(Co-)IP

CRL

Da

$\Delta$-F-box

DMEM

DMSO

dNTP

DTT

DUB

E.coli

ECL

EDTA

EGTA

FCS

G

GAP

GEF

GFP amino acids

adenine in context of DNA/RNA

adenosine-5'-diphosphate

ammonium persulfate

adenosine-5'-triphosphate

bovine serum albumine

cytosine in context of DNA and RNA

Carboxyl-, in context of proteins

complementary DNA

Calf Intestine Phosphatase

(co-)immunoprecipitation

cullin-RING based Ubiquitin E3 ligase

Dalton

$$
\text { deletion of the F-box domain }
$$

Dulbeccos's modified Eagles medium

dimethyl sulfoxide

2'-desoxynucleoside-5'-triphosphate

dithiotreitol

deubiquitinase, deubiquitylating enzyme

Escherichia coli

enhanced chemical luminescence

ethylenediaminetetraacetic acid

ethylene glycol tetraacetic acid

fetal calf serum

guanine, in context of DNA and RNA

GTPase-activating protein

guanine nucleotide exchange factor

green fluorescence protein 
GST glutathione-S-transferase

GTP guanosine-5'-triphosphate

HA hemagglutinin

$\mathrm{HCl} \quad$ hydrochloric acid

HECT $\quad$ Homologous to the E66-AP Carboxyl Terminus

HEPES [4-(2-hydroxyethyl)-1-piperazine]ethanesulfonic acid

His- hexahistidine tag

IF immunofluorescence

IgG immunoglobuline $\mathrm{G}$

IPTG isopropyl-beta-D-thiogalactopyranoside

LB Luria-Bertani

LIR LC3 interaction region

MS mass spectrometry

$\mathrm{N}-\quad$ Amino-, in context of protein

NCS newborn calf serum

NEM N-ethylmaleimide

NP-40 nonyl phenoxypolyethoxylethanol

$\mathrm{OH} \quad$ Hydroxyl

PAGE polyacrylamide gel electrophoresis

PBS phosphate buffered saline

PCR polymerase chain reaction

PMSF phenylmethylsulphonyl fluoride

RIPA radio immunoprecipitation assay

RNA ribonucleic acid

RNAi RNA interference

RING Really Interesting New Gene

S.cerevisiae Saccharomyces cerevisiae

SCF Skp1-Cul1-F-box

SDS sodium dodecyl sulfate

siRNA small interfering RNA

SRS substrate recognition subunit

$\mathrm{T} \quad$ thymine, in context of DNA

TAE Tris/ acetate / EDTA 
TE Tris / EDTA

TEMED tetramethylethylenediamine

Tris tris(hydroxymethyl)aminomethane

Triton-X100 4-octylphenol polyethoxylate

Tween-20 polyoxylethylene (20) sorbitan monolaurate

$\mathrm{U} \quad$ uracile, in context of RNA

Ubc Ubiquitin conjugating enzyme

UBD Ubiquitin binding domain

$\mathrm{v} / \mathrm{v} \quad$ volume per volume

w/v weight per volume

wt wildtype

\section{Physical units}

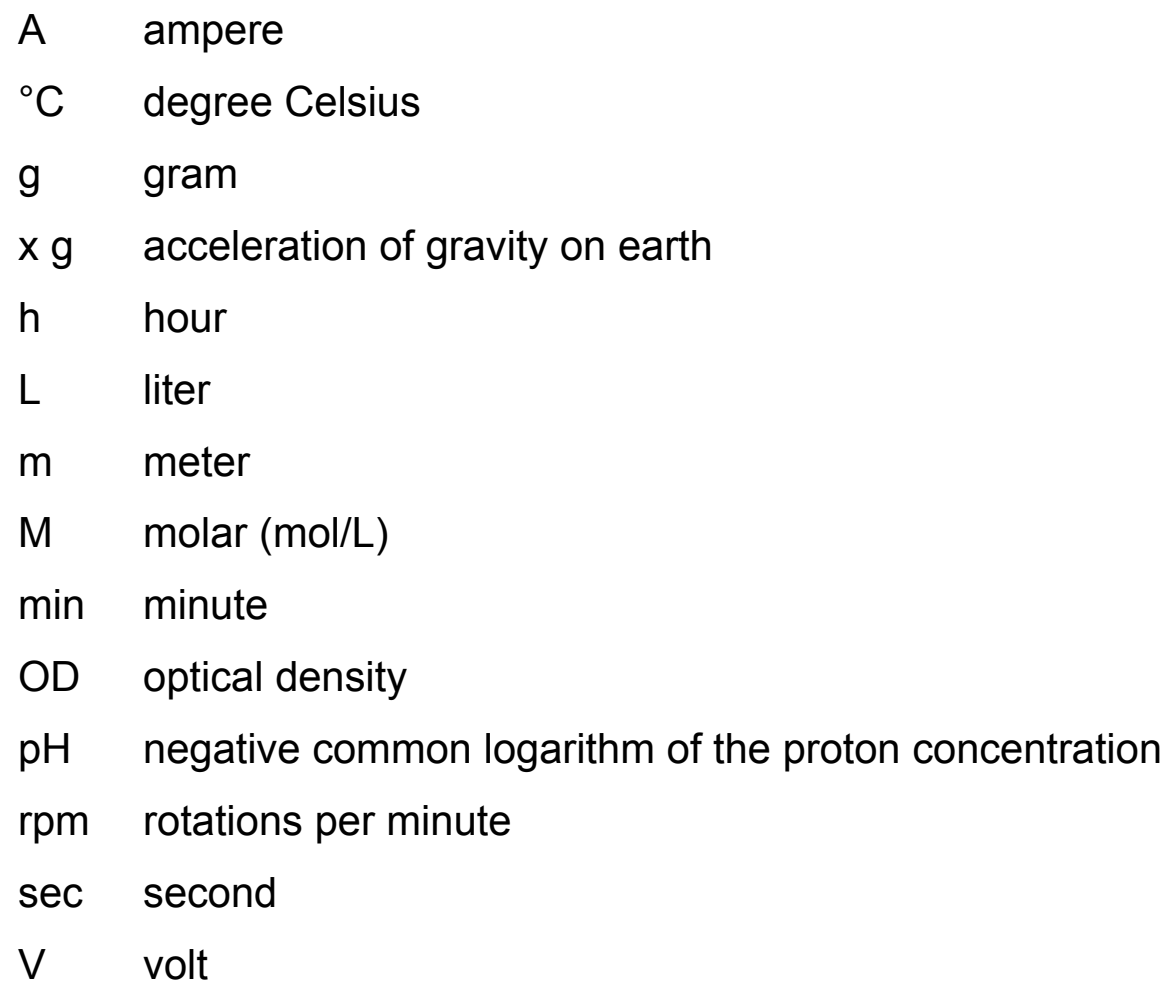

\section{Prefixes}

k kilo- $10^{3}$

c centi- $10^{-2}$

$\mathrm{m} \quad$ mili- $10^{-3}$

$\mu \quad$ micro- $10^{-6}$ 
n nano- $10^{-9}$

p pico- $10^{-12}$

\section{Code for amino acids}
A Ala alanine
C Cys cysteine
D Asp aspartate
E Glu glutamate
F Phe phenylalanine
G Gly glycine
$\mathrm{H} \quad \mathrm{His}$ histidine
I Iso isoleucine
$\mathrm{K}$ Lys lysine
L Leu leucine
M Met methionine
$\mathrm{N}$ Asn asparagine
P Pro proline
Q Gln glutamine
$\mathrm{R}$ Arg arginine
S Ser serine
$\mathrm{T} \quad \mathrm{Thr}$ threonine
V Val valine
W Trp tryptophane
$Y \quad$ Tyr tyrosine
$\mathrm{X} \quad$ any amino acid
$\psi \quad$ bulky hydrophobic residue 


\section{CURRICULUM VITAE}

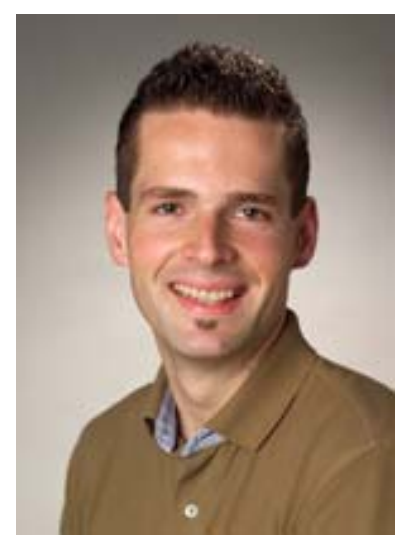

Education
Name:

Date of birth:

Place of birth:

Nationality:

Contact address:

Phone:

FAX:

Email:
Achim Werner January $11^{\text {th }}, 1982$

Kassel, Germany

German

DKFZ-ZMBH Alliance

University of Heidelberg Im Neuenheimer Feld 282

D-69120 Heidelberg +49 (6221) 54-6879

+49(6221) 54-5893

a.werner@zmbh.uniheidelberg.de since Apr 2009

since Oct 2006

2005-2006

$2004-2005$

$2002-2005$

$2001-2002$

$1999-2001$
PhD Thesis, DKFZ-ZMBH Alliance, University of Heidelberg:

- Continuation of PhD project started in Göttingen after lab move

PhD Thesis, Department of Biochemistry, University of Göttingen:

- Thesis Title: "Mechanistic and functional characterization of Fbw5"

- Thesis Committee: Prof. Frauke Melchior $\left(1^{\text {st }}\right.$ supervisor), Prof. Reinhard Lührmann (Max Planck Institute of Biophysical Chemistry, Göttingen), Prof. Gerhard Braus (University of Göttingen)

MSc/PhD Program Molecular Biology, University of Göttingen:

- International Max Planck Research School Molecular Biology

- MSc examination grade point average: 1.4 (excellent)

BSc thesis, Department of Chemistry, University of Lübeck:

- Thesis Title: "Purification and functional characterization of rat ST3Galll"

- Grade: 1.0 (very good)

- supervisor: Prof. Dr. Thomas Peters

BSc Program Molecular Life Science, University of Lübeck:

- Graduation Certificate: BSc

- Grade Point average: 1.5 (very good)

Military Service, Pommernkaserne, Wolfhagen

Secondary School, Ursulinenschule, Fritzlar:

- Graduation Certificate: Abitur

- Grade point average: 1.2 (very good) 


\section{Scientific Activities}
May - Jun 2006 Lab Project: "In vitro reconstitution of chromatin", Laboratory of Chromatin Biochemistry, Max Planck Institute for Biophysical Chemistry, Göttingen (Supervisor: Dr. Wolfgang Fischle)
Mar - Apr 2006 Lab project: "Investigation of the role of Vti1a in homotypic early endosomal fusion", Department of Neurobiology, Max Planck Institute for Biophysical Chemistry, Göttingen (Supervisor: Dr. Silvio Rizzoli)
Jan - Feb 2006 Lab Project: "Fluorescence and crystallographic studies on human $\mathrm{C}_{\mathrm{a}}$-Formylglycine-Generating Enzyme and its paralog pFGE", Department of Molecular Structural Biology, University of Göttingen (Supervisor: Dr. Markus Rudolph)

Teaching

\begin{tabular}{|c|c|}
\hline 2009 - present & $\begin{array}{l}\text { Supervising tutor: biochemical training for Life Science M.Sc. } \\
\text { students, University of Heidelberg }\end{array}$ \\
\hline $2006-2009$ & $\begin{array}{l}\text { Supervising tutor: biochemical training for medical students, } \\
\text { University of Göttingen }\end{array}$ \\
\hline $2006-2008$ & $\begin{array}{l}\text { Tutor within the framework of biochemical lectures for the } \\
\text { „International Max Planck Research School Molecular Biology“ }\end{array}$ \\
\hline
\end{tabular}

\section{Scholarships / Prizes}

Sep 2009

$3^{\text {rd }}$ Student Talk Prize, $6^{\text {th }}$ International PhD Symposium "Horizons in Molecular Biology", Göttingen

2005 - $2006 \quad$ Stipend International Max Planck Research School

2005 - $2006 \quad$ Masters Scholarship of the "Studienstiftung des deutschen Volkes" (German National Merit Foundation)

2003 - $2006 \quad$ Undergraduate and Masters Scholarship of the "Stiftung der Deutschen Wirtschaft " (Foundation of German Business)

1998 - 1999 Scholarship of the German Bundestag and the American Congress:

Participation in the $15^{\text {th }}$ German-American Youth Exchange

\section{Activities / Honorary work}

$2006-2009 \quad$ Organization of the $4^{\text {th }}$ and $5^{\text {th }}$ International PhD Student Symposium "Horizons in Molecular Biology" (http://www.horizons.uni-goettingen.de/) Organization of the $1^{\text {st }}$ and $2^{\text {nd }}$ "Horizons Career Fair" (http://www.horizons.uni-goettingen.de/)

1999 - present Honorary work for Experiment e.V. (non-profit organization with charitable status with the goal to exchange between people of all cultures, religions and age groups) 